\title{
Physiological functions of the adrenocortical circadian clock
}

\author{
Dissertation \\ for the award of the degree \\ "Doctor rerum naturalium" \\ Division of Mathematics and Natural Sciences \\ of the Georg-August-Universität Göttingen \\ submitted by \\ Alexei Leliavski \\ from Minsk, Belarus
}

Göttingen, 2013 


\section{Thesis Committee:}

Prof. Dr. Henrik Oster (reviewer)

Chronophysiology Group, Medical Department I, University of Lübeck

Prof. Dr. Michael Kessel (reviewer)

Department of Developmental Biology, Max Planck Institute for Biophysical Chemistry, Göttingen

Prof. Dr. Andreas Wodarz

Department of Anatomy and Cell Biology, Göttingen Center for Molecular Biosciences (GZMB), University of Göttingen

Date of oral examination: 13.02.2014 


\section{Declaration}

Herewith, I confirm that I have written the present $\mathrm{PhD}$ thesis independently and with no other sources and aids than quoted.

Lübeck, December 2013

Alexei Leliavski 
To my parents. 


\section{Table of Contents}

Declaration 3

Summary 8

Chapter 1. Introduction. 10

1.1. Circadian rhythms. Properties of the circadian clock 10

1.1.1. Sustainability 10

1.1.2. Entrainment

1.1.3. Temperature compensation

1.2. The molecular clockwork

1.3. The anatomical basis of circadian rhythms: central and peripheral clocks

1.3.1. The central clock 14

1.3.2. The circadian clocks in peripheral tissues 15

1.3.3. Communication between central and peripheral clocks 16

1.4. Circadian rhythms and disease: clinical applications 18

1.5. GCs, stress response, and the circadian clock 19

1.5.1. Anatomy and physiology of the adrenal gland. 20

1.5.2. Regulation of GC production: Anatomy of the HPA axis

1.5.3. Regulation of GC production: Autonomic innervation 22

1.5.4. Regulation of GC sensitivity: from release to action 23

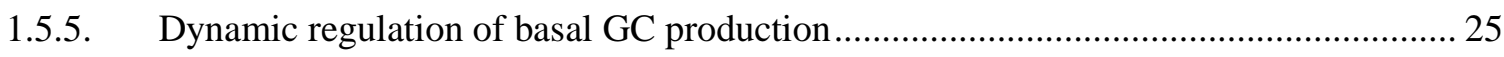

1.5.6. Circadian GC rhythms and synchronization of tissue clocks .......................................... 30

1.5.7. Circadian GC oscillations and rhythmic regulation of tissue functions ........................ 32

1.5.8. Dynamics: regulation of GC response to acute stress ................................................ 33 
1.5.9. The circadian clock and stress response

1.6. Publication: "Physiology of the adrenal and liver circadian clocks"

1.7. Aims of the work 56

Chapter 2. Results 57

2.1. Publication „Impaired Glucocorticoid Production and Response to Stress in Arntl-Deficient Male Mice“. 57

2.2. Generation and evaluation of adrenocortical clock-deficient (ACD) mice 72

2.2.1. Generation of conditional knockout mice deficient in adrenocortical circadian clock 72

2.2.2. Circadian hormone production in the adrenocortical clock-deficient mice 75

2.2.3. Circadian behavioral resynchronization in response to a jet lag in the adrenocortical clockdeficient mice 77

Chapter 3. Discussion and Outlook 80

3.1. Conditional disruption of circadian clocks in the adrenal cortex: methodological issues. 80

3.2. The adrenocortical clock and hormonal rhythms 82

3.4. The adrenocortical clock and stress responses 84

3.5. The adrenocortical clock and the synchronization of circadian clocks ... 85

3.6. Towards a comprehensive understanding of the mammalian circadian organization: the adrenocortical clock and the stability of the circadian timing system 86

3.7. Outlook 89

Chapter 4. Materials and Methods 90

4.1. Animal experiments 90

4.1.1. Animal housing and breeding 90

4.1.2. Tissue, blood and feces collection 90

4.1.3. Behavioral tests 90 
4.1.4. Dexamethasone suppression test

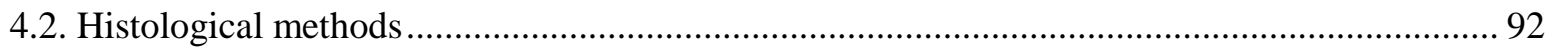

4.2.1. Hematoxylin-eosin staining and histological analysis of adrenal glands .......................... 92

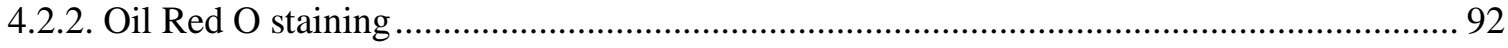

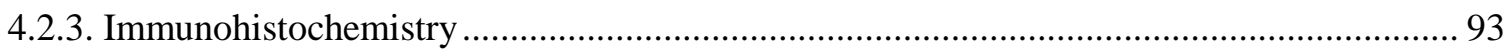

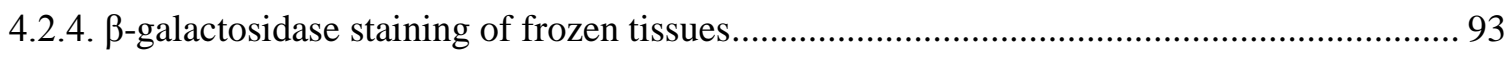

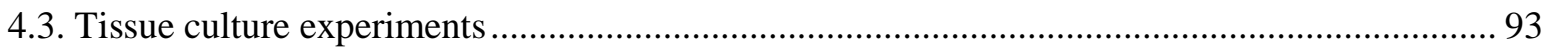

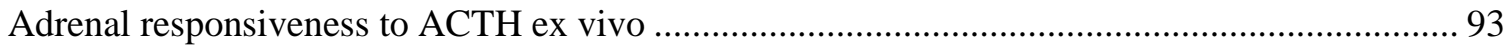

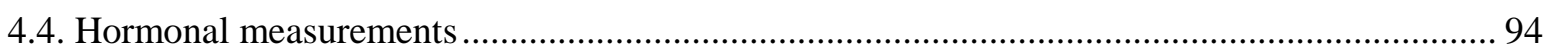

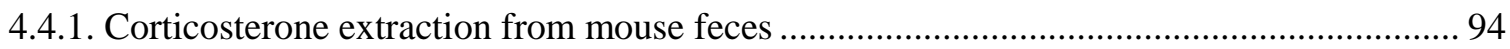

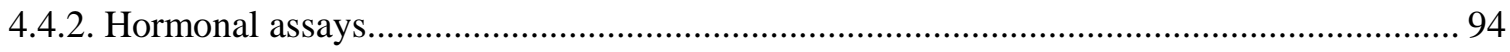

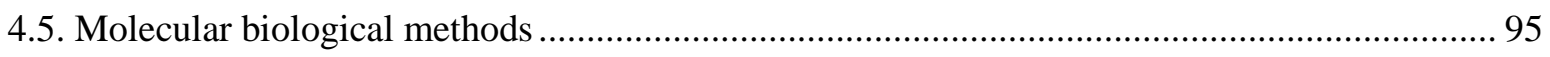

4.5.1. Quantitative reverse transcription polymerase chain reaction (qRT-PCR) ........................ 95

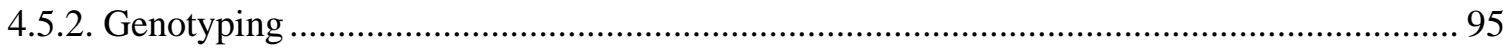

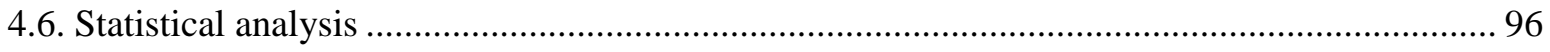

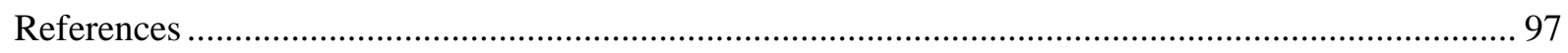

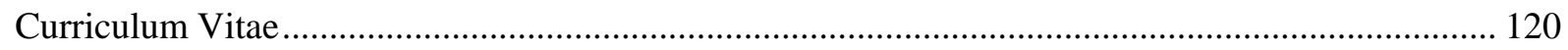

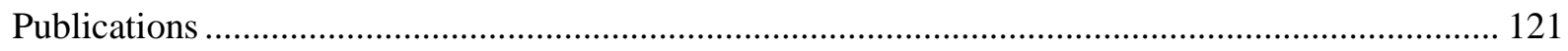

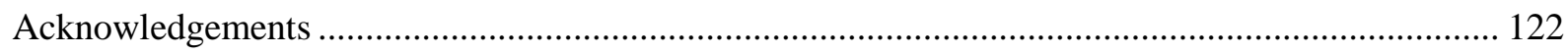




\section{Summary}

In the course of evolution most organisms have evolved endogenous circadian clocks that help them to anticipate daily environmental changes in light, temperature and food availability and therefore adjust physiology and behavior in a more efficient manner. The mammalian genome encodes a number of dedicated clock proteins, which coordinate the rhythmic transcription and translation of hundreds of genes in almost every cell of the body. The transcriptional activator BMAL1 (also known as ARNTL or MOP3) is at the core of this molecular clockwork and indispensable for circadian clock function. Information about external time, so called Zeitgeber input, is integrated by a circadian master pacemaker in the hypothalamic suprachiasmatic nuclei $(\mathrm{SCN})$ that synchronizes subordinate clocks in other brain regions and peripheral tissues via different routes including hormonal signals, autonomic innervation, and regulation of the sleep-wake cycle. Glucocorticoid (GC) hormones (mainly cortisol in humans and corticosterone (CORT) in rodents) produced by the adrenal cortex in a rhythmic fashion are essential for the coordination of responses to stress, but also act as systemic synchronizers of circadian rhythms in various tissues. It has previously been shown that adrenocortical clocks modulate the sensitivity of the steroidogenic machinery to external stimuli. Their role in the regulation of stress responses, however, remains unclear. To close this gap and to study adrenocortical clock function in vivo, two genetic mouse models with full and conditional deletion of the Bmall gene were used in this study.

In the first part of the thesis I studied GC production and stress responses in Bmall-deficient mice. Under unstressed conditions Bmall ${ }^{-/}$mutants suffer from hypocortisolism, associated with impaired adrenal responsiveness to adrenocorticotropin (ACTH) and down-regulated transcription of genes involved in cholesterol transport and steroidogenesis in the adrenal gland, such as Star, Stard4, Ldlr and Por. Bmall-deficient mice show reduced GC responses to acute stress, but preserved ACTH responses. Furthermore, they develop behavioral resistance to acute and sub-chronic stressors, as shown using forced swim, tail suspension and sucrose preference tests. These data suggest that the clock gene Bmall regulates circadian and acute secretion of GCs by the adrenal gland and contributes to behavioral resistance to stress, probably via its effects on adrenocortical function.

The second part of the thesis focuses on the generation of conditional knockout mice that lack a functional circadian clock specifically in adrenocortical cells. For this purpose Bmall flffl mice were cross-bred with Cyp 1 1al-Cre mice that express the CRE recombinase in steroid-producing cells of the adrenal gland and the gonads. Immunohistochemical stainings reveal high efficiency of BMAL1 deletion in the adrenal cortex of Cypllal ${ }^{\mathrm{Cre} /+}$;Bmall ${ }^{\mathrm{flfl} f}$ (ACD) mice compared to Cypllal ${ }^{\mathrm{Cre} / \mathrm{+}}$ controls. Moreover, abolished rhythms in the expression of clock and clock-controlled genes in the adrenal cortex, but not in the kidney, of ACD mice confirm a disrupted functionality of the 
adrenocortical clock. However, circadian rhythms of CORT and aldosterone are not significantly altered in ACD mice kept in constant darkness. This suggests that the adrenocortical clock itself is dispensable for maintenance of circadian GC rhythms under unstressed conditions. Considering that GCs play a crucial role in the entrainment of circadian clocks, I analyzed behavioral responses of ACD and control mice to a rapid shift of the light-dark cycle. After a 6-hour phase advance the photic re-entrainment of locomotor activity of ACD mice occurs significantly faster compared to control mice, indicating that the adrenocortical clock contributes to the robustness of the circadian system under conditions of persistent external noise.

In conclusion, this study shows that the adrenocortical clock plays a role in regulation of hormonal and behavioral responses to stress and contributes to the phase stability of the circadian system. Since many people live under conditions of regular Zeitgeber contamination, e.g. during shift work, manipulation of the adrenocortical clock may help buffering our endogenous clocks against exogenous perturbation. On the other hand, suppressing the stabilizing effect of the adrenocortical clock could speed up adaption of the circadian system when this is wanted, such as during jetlag. 


\section{Chapter 1. Introduction}

\subsection{Circadian rhythms. Properties of the circadian clock}

Day-night rhythms in physiology of many organisms have been recognized for centuries. However it is much less obvious that these daily cycles are not merely induced, or driven, by recurring changes of light and temperature. Remarkably, the external cues generated by the rotation of the Earth around its axis serve only as rhythmic synchronizers of endogenous biological clocks. These circadian clocks (from the Latin circa diem - "about a day") can independently generate robust rhythms in gene expression, protein activity and hence functional outputs with a period of approximately 24 hours. The circadian clock is encoded in the genome and based on transcriptional-translational feedback. In mammals, likely every cell of the body contains an active circadian clock.

Three key properties can be attributed to a bona fide circadian clock: an endogenous ability to sustain a nearly 24-h rhythm, responsiveness to entraining signals, and relative stability of endogenous period to fluctuations of ambient temperature.

\subsubsection{Sustainability}

A key property of a biological clock is its ability to maintain oscillations in the absence of external rhythmic signals. The first well known evidence that a biological organism can sustain a rhythmic behavior dates back to a study of the French astronomer Jean-Jacques d'Ortous de Mairan who found, in 1729, that daily rhythmicity in leaf movement of Mimosa pudica was preserved even when the plant was kept in a dark place (Daan, 2010). Since then, self-sustained rhythms in physiology and behavior have been reported in species almost from every branch of the evolutionary tree. Despite the fact that an endogenous period usually differs from precisely 24 hours, doubts on the intrinsic nature of the circadian oscillations persisted for a long time, until a firm and rigorous basis of circadian biology was established from many convincing experiments conducted by Jürgen Aschoff, Colin Pittendrigh and other notable chronobiologists (Daan, 2010). One of the most extraordinary ways to confirm the endogenous nature of circadian rhythms was an automated recording of the photoaccumulation rhythm in the unicellular eukaryote Chlamydomonas reinhardtii aboard a spacecraft orbiting the earth every 90 min (Mergenhagen and Mergenhagen, 1987). The rhythms continued for at least 6 days in microgravity without any terrestrial cue of the time of day, showing conclusively that the circadian clock can run independently from daily cycles of gravity, magnetism, cosmic ray irradiation etc. (Mittag et al., 2005). 


\subsubsection{Entrainment}

Entrainment can be defined as a synchronization of a self-sustained oscillator by a rhythmic external signal (Daan, 2010; Golombek and Rosenstein, 2010; McEwen and Gianaros, 2010; Roenneberg et al., 2003). The strongest external cues able to entrain the circadian clock, or, according to terminology originally proposed by J. Aschoff, Zeitgebers (German "time givers"), include light, temperature and feeding, but many other signals can also serve as Zeitgebers (e.g. social interactions, exercise, sounds) (Aschoff et al., 1971; Menaker and Eskin, 1966; Mistlberger and Skene, 2005). A range of entrainment is defined by strength (amplitude) and period length of the Zeitgeber signal rhythms. Circadian clocks can be entrained by a Zeitgeber only when the period of the latter is sufficiently close to the natural period of the oscillator (from 23 to $25 \mathrm{~h}$ for most mammals). In the entrainment regime, the period of the circadian clock becomes equal to the Zeitgeber period, and the phase difference between the oscillator and the entrainment signal is fixed (Mondragon-Palomino et al., 2011; Roenneberg et al., 2003).

\subsubsection{Temperature compensation}

In lower, poikilothermic organisms temperature fluctuations may affect rates of chemical reactions and therefore alter functionality of the circadian clock. For this reason specific mechanisms were evolved to confer a relative resistance of circadian clocks to temperature changes (so called temperature compensation). Independence of the circadian rhythm in leaf movement from temperature fluctuations was demonstrated for the first time in the 18th century, soon after de Mairan's observation(Golombek and Rosenstein, 2010), and later C. Pittendrigh confirmed the existence of this phenomenon in animals, revealing temperature independence of circadian eclosion rhythms in Drosophila (Pittendrigh, 1954). Of note, large temperature changes still do affect circadian period length, but these changes are very minor, compared to most biological systems. For example, C. Johnson and colleagues showed that the cyanobacterial circadian clock changes its endogenous period from $25.5 \mathrm{~h}$ at $25^{\circ} \mathrm{C}$ to $23 \mathrm{~h}$ at $36^{\circ} \mathrm{C}$, which corresponds to a temperature coefficient $\mathrm{Q}_{10}$ of 1.1 (Kondo et al., 1993). This is far from that of most biological processes, for which the $\mathrm{Q}_{10}$ values range from 2 to 3 .

\subsection{The molecular clockwork}

The first "clock" gene, Period, was characterized as mutations of a Drosophila gene that affected the period length of circadian behavioral rhythms in locomotion and eclosion (Konopka and Benzer, 1971). Since then, forward genetic screens in vivo and in cell lines identified dozens and even hundreds of genes, the down-regulation of which leads to the impairment of clock function (Zhang and Kay, 2010). 
It is now well established that the molecular circadian clock in the majority of organisms is based on interlocked transcriptional-translational regulatory feedback loops. This is in contrast to many other biological oscillators that normally do not require gene transcription as a principal matter for rhythm generation (Goldbeter, 2002). In mammals (Figure 1), the bHLH-PAS transcriptional activators CLOCK (or NPAS2) and BMAL1 heterodimerize and induce transcription of many target genes in the morning by binding to specific DNA elements, called E- and E'-boxes. Among the activated genes are those that encode the inhibitors of CLOCK/BMAL1 transcriptional activity - two Period (Per1, Per2) and two Cryptochrome (Cry1, Cry2) genes. Further PER/CRY-mediated suppression of CLOCK/BMAL1 activity down-regulates the strength of the negative feedback and therefore reinitiates the circadian cycle (Buhr and Takahashi, 2013; Oster, 2006).

Figure 1. Molecular organization of the mammalian circadian clock. Modified from (Oster, 2006).

TTL-transcriptional-translational feedback loop, CCGs—clock controlled genes. CREB-CAMP response element (CRE)-binding protein.

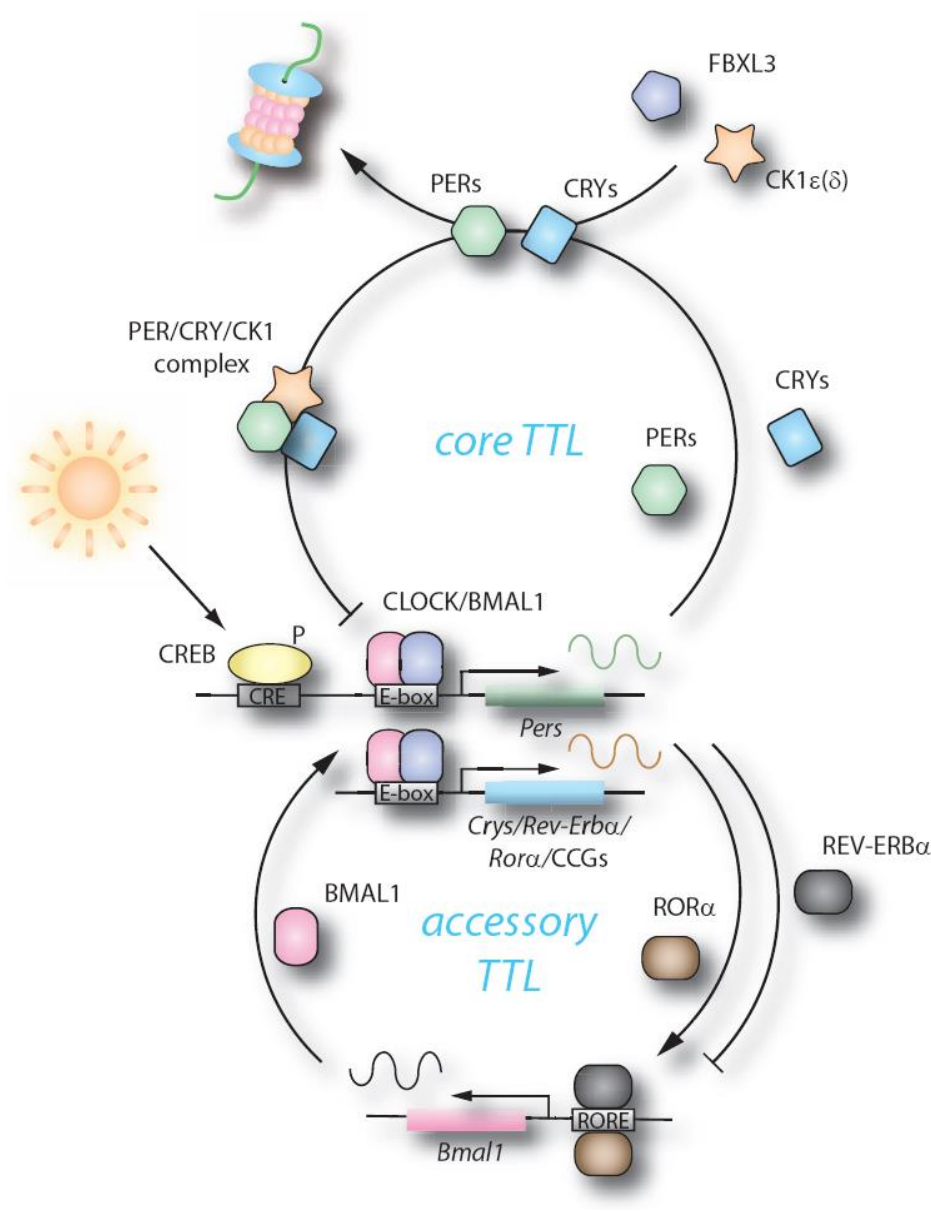

To ensure robustness and flexibility of the system, the dynamics of the positive and negative limbs of the circadian feedback are under complex control, including posttranscriptional (e.g. (Fustin et al., 2013)) and posttranslational modifications (Reischl and Kramer, 2011) as well as chromatin remodeling (Sahar and Sassone-Corsi, 2013).

The period length is finely adjusted through the regulation of stability of the PER and CRY proteins. This is achieved by posttranslational modifications ((Buhr and Takahashi, 2013; Reischl and Kramer, 
2011) and refs within). For instance, phosphorylation of the PER proteins by two casein kinases, CK1 $1 \varepsilon$ and $\mathrm{CK} 1 \delta$, leads to their ubiquitination by $\beta \operatorname{TrCP}$ and subsequent proteasomal degradation. Similarly, CRY proteins phosphorylated by specific kinases (AMPK1 phosphorylates CRY1, whereas CRY2 is phosphorylated sequentially by DYRK1A and GSK-3 $\beta$ kinases) are then polyubiquitinated by the E3 ubiquitin ligase FBXL3 and degraded in the proteasome. In contrast, ubiquitination by another E3 ligase, FBXL21, stabilizes CRY proteins by competing with the destabilizing action of FBXL3.

Rhythmic binding of the CLOCK/BMAL1 complex to E-box-containing transcriptional elements occurs on a genome-wide scale and is associated with dynamic chromatin remodeling, including rhythmic recruitment of RNA polymerase II and coactivators and circadian cycling of chromatin modifications such as histone acetylation and methylation (Koike et al., 2012). The CLOCK and BMAL1 proteins can rhythmically recruit chromatin modifiers such as the methyltransferase MLL1 and the deacetylases HDAC3 and SIRT1 and therefore alter chromatin accessibility at target genes (Sahar and Sassone-Corsi, 2013). Moreover, the CLOCK protein per se possesses a histone acetyl transferase activity (Doi et al., 2006).

In addition to the "classical" feedback loops, the mammalian molecular clock includes accessory regulatory limbs that are based on clock-controlled DNA binding elements different from E-boxes. $\mathrm{REV}-\mathrm{ERB} \alpha / \beta$ and ROR $\alpha$ proteins compete for binding to RORE sequences in regulatory regions of many genes and, importantly, in the promoter of the Bmall gene, acting, respectively, as inhibitors or activators of its transcription (Preitner et al., 2002; Sato et al., 2004). REV-ERB $\alpha / \beta$-mediated regulation is indispensable for proper functioning of the circadian clock, since an inducible deletion of both genes in adult mice leads to severe disruption of locomotor behavior in constant darkness (Cho et al., 2012). Another potential transcriptional loop is made of additional clock-controlled transcription factors including PAR bZIP proteins (DBP, TEF, HLFs), KLF10 and E4BP4, which contain E-box elements or ROREs in their promoters (Asher and Schibler, 2011). These factors are dispensable for basic clock function, but still important for sustaining robust period of oscillations (Liu et al., 2008). Together, different combinations of three clock-controlled elements (E-boxes, D-boxes and ROREs) control the temporal dynamics of the circadian clockwork and clock-controlled genes (Ueda et al., 2005).

Importantly, genetic inactivation of core clock genes results in dramatic alterations of circadian locomotor rhythms in vivo. Robustness of the circadian clock is supported by the fact that Bmall (or Arntl) is the only known clock gene in mammals, knockout of which leads to behavioral arrhythmicity (Bunger et al., 2000). Due to functional redundancy, in vivo circadian clock ablation normally occurs when at least two functionally similar genes are deleted, as shown in Clock/Npas2, Cry1/Cry2, Per1/Per2, and Per2/Cryl double knockout mice (DeBruyne et al., 2007a; Oster et al., 2006b; van der Horst et al., 1999; Zheng et al., 2001). 


\subsection{The anatomical basis of circadian rhythms: central and peripheral clocks}

\subsubsection{The central clock}

Molecular clocks are present in most tissues and cells, but their functional roles of maintaining circadian rhythms of physiology and behavior seem to be not equal. The principal anatomical region responsible for generation of circadian rhythms was located to the suprachiasmatic nuclei (SCN) in the hypothalamus (reviewed in (Welsh et al., 2010)). Lesion studies showed that in the absence of the SCN animals are devoid of circadian behavioral and hormonal rhythms in constant darkness (Abe et al., 1979; Moore and Eichler, 1972; Stephan and Zucker, 1972). In accordance, recent genetic ablation experiments confirmed that conditional inactivation of the circadian clock in SCN neurons leads to behavioral arrhythmicity in constant conditions, similar to what is seen in Bmall-deficient mice (Husse et al., 2011). In addition, elegant experiments using SCN transplantations between wild-type and circadian mutant hamsters demonstrated that the SCN determines the period of endogenous behavioral rhythms (Ralph et al., 1990). Finally, wild-type SCN grafts can restore circadian behavioral rhythms when transplanted into arrhythmic Cry1/Cry2-deficient mice (Sujino et al., 2003).

A distinct feature of the SCN clock is its ability to generate self-sustained, coherent and robust oscillations over a long time (virtually during over the whole life span of an animal) in the absence of synchronizing light cues. Circadian clocks in different SCN neurons are coupled together via synaptic communication between neurons, gap junctions, and paracrine interactions via neuropeptides such as VIP (Welsh et al., 2010). Importantly, intercellular coupling between SCN neurons is essential to confer robustness of the oscillations against genetic perturbations, as shown for Perl, Cryl, and Bmall deficient SCN explants (Ko et al., 2010; Liu et al., 2007).

The SCN is important for light-dependent synchronization of diurnal rhythms in physiology and behavior. Sitting on the top of the optic chiasm, it receives light information via the retina of the eye from a subpopulation of non-image-forming photoreceptors, so called intrinsically photosensitive retinal ganglion cells (ipRGCs), which express the light-sensitive pigment melanopsin (Schmidt et al., 2011). Animals that lack ipRGCs still retain pattern vision but have extensive deficits in their pupillary reflex to light and circadian photoentrainment (Guler et al., 2008). The ipRGCs activated by light reset the circadian clocks in SCN neurons through glutamate/PACAP-dependent signaling pathways which culminates in induction of Per and immediate early genes (Albrecht et al., 1997; Dibner et al., 2010). Additionally, the SCN receives non-photic information from the geniculohypothalamic tract and serotoninergic neurons of the raphe nuclei. 


\subsubsection{The circadian clocks in peripheral tissues}

Most peripheral tissues and brain regions outside the SCN were shown to contain circadian oscillators that drive circadian rhythms in the transcription of hundreds of genes (normally from 5 to $10 \%$ of genes expressed in a given tissue) (Doherty and Kay, 2010). Generation of circadian clock-controlled luciferase reporter rodents such as Per2::luc mice and Perl-luc rats provided a powerful genetic tool for real-time registration of circadian oscillations in various tissues and allowed to demonstrate selfsustained circadian rhythms of gene expression in culture over several weeks (Yamazaki et al., 2000; Yoo et al., 2004) (Figure 2). In accordance, circadian rhythms in tissue physiology have been consistently observed for cultured retina (Tosini and Menaker, 1996), adrenal glands (Andrews and Folk, 1964), lymphocytes (Gamaleia et al., 1988) and other tissues. The dampening of circadian oscillations in culture is related to desynchrony between individual cell rhythms, rather than gradual loss of the intrinsic capacity to maintain oscillations (Welsh et al., 2004). In vivo, a significant dispersion of phase in peripheral oscillators was observed in SCN-lesioned Per2::luc mice, both within and among animals (Yoo et al., 2004). These findings provide strong evidence that the central SCN clock serves mainly as a master synchronizer of largely independent rhythms in individual cells and does not drive the circadian rhythms in the rest of the body (Dibner et al., 2010).

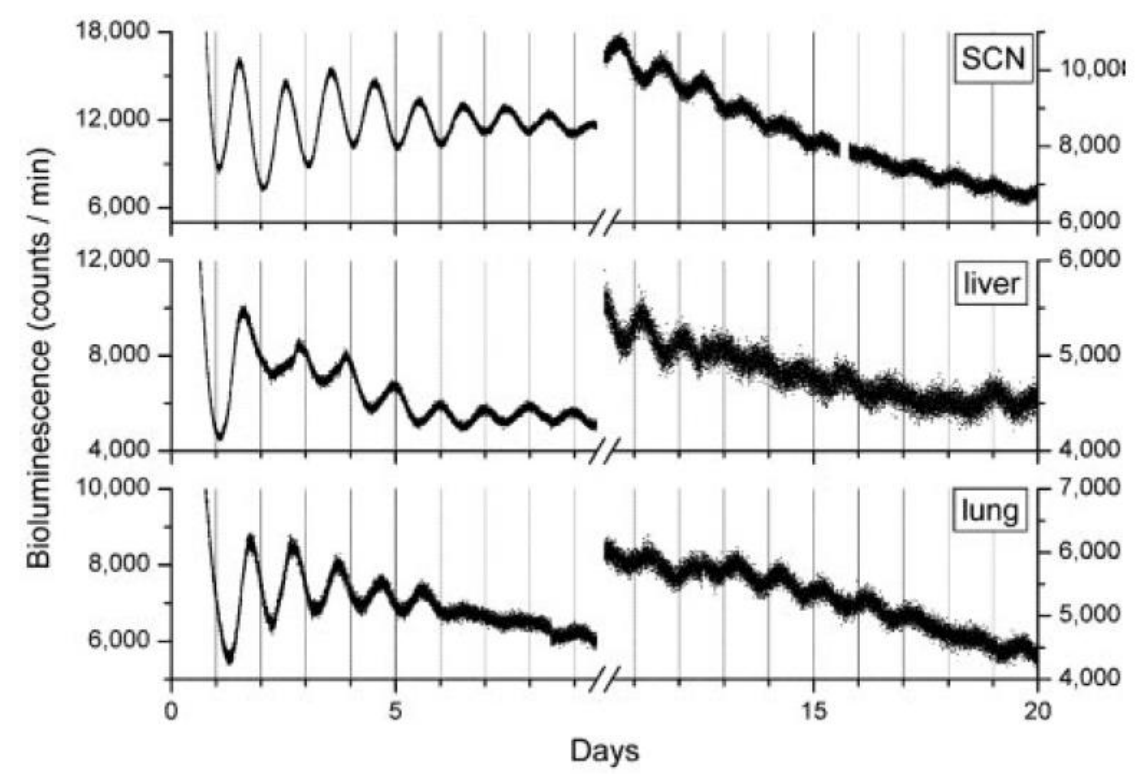

Figure 2. Long-term persistent circadian rhythms of bioluminescence in peripheral tissues of Per2::luc mice. From (Yoo et al., 2004).

Physiological significance of some peripheral circadian clocks has been evaluated using conditional knockout models. For example, mice with liver-specific ablation of Bmall exhibit hypoglycemia restricted to the fasting phase of the daily feeding cycle, exaggerated glucose clearance, and loss of 
rhythmic expression of hepatic glucose regulatory genes (Lamia et al., 2008). A diabetes-like phenotype including impaired glucose tolerance and hypoinsulinemia was observed in mice with conditional ablation of the pancreatic circadian clock (Marcheva et al., 2010). Finally, deletion of Bmall in myeloid cells including monocytes and macrophages exacerbates pathologies associated with acute and chronic inflammation, both of infectious (Listeria infection) and non-infectious (metabolic inflammation) origin (Nguyen et al., 2013).

\subsubsection{Communication between central and peripheral clocks}

SCN neurons convey the timing information to other organs and tissues via distinct routes (Dibner et al., 2010) (Figure 3). The SCN can target peripheral organs directly, by releasing humoral factors or sending neural projections to the autonomic nervous system (ANS). Restoration of locomotor, but not hormonal, rhythms was observed in SCN lesioned animals after transplantation of fetal SCN tissue encapsulated in a semi-permeable membrane, which prevents axonal growth (Meyer-Bernstein et al., 1999; Silver et al., 1996). Humoral factors secreted from the SCN which may be responsible for regulation of behavioral periodicity include $\mathrm{TGF} \alpha$, prokinecitin-2 and cardiotropin-like cytokine (Cheng et al., 2002; Kramer et al., 2001; Kraves and Weitz, 2006).

The SCN clock controls brain areas responsible for regulation of hormonal production, for instance, paraventricular nucleus (PVN) neurons, which activate the HPA axis and stimulate GC synthesis by adrenal glands (section 1.5.5.). Hormones can therefore serve as synchronizers of peripheral clocks. Complementary, the SCN acts through the ANS to synchronize circadian rhythms in peripheral organs, as shown for liver, heart, pancreas, adrenal gland, and kidney (Buijs et al., 2013), and some brain regions, for example the melatonin-producing pituitary gland (Pevet and Challet, 2011). SCNANS connections are important for circadian modulation of blood pressure and heart rate (Scheer et al., 2001) as well as for appropriate daily timing of glucose production and insulin sensitivity (Cailotto et al., 2005; la Fleur et al., 2001). Sympathetic denervation by 6-hydroxydopamine flattens the daily rhythm of Perl and Per2 gene expression in the liver, whereas electrical stimulation of the sympathetic nerve increases hepatic Perl expression in vivo (Terazono et al., 2003). 


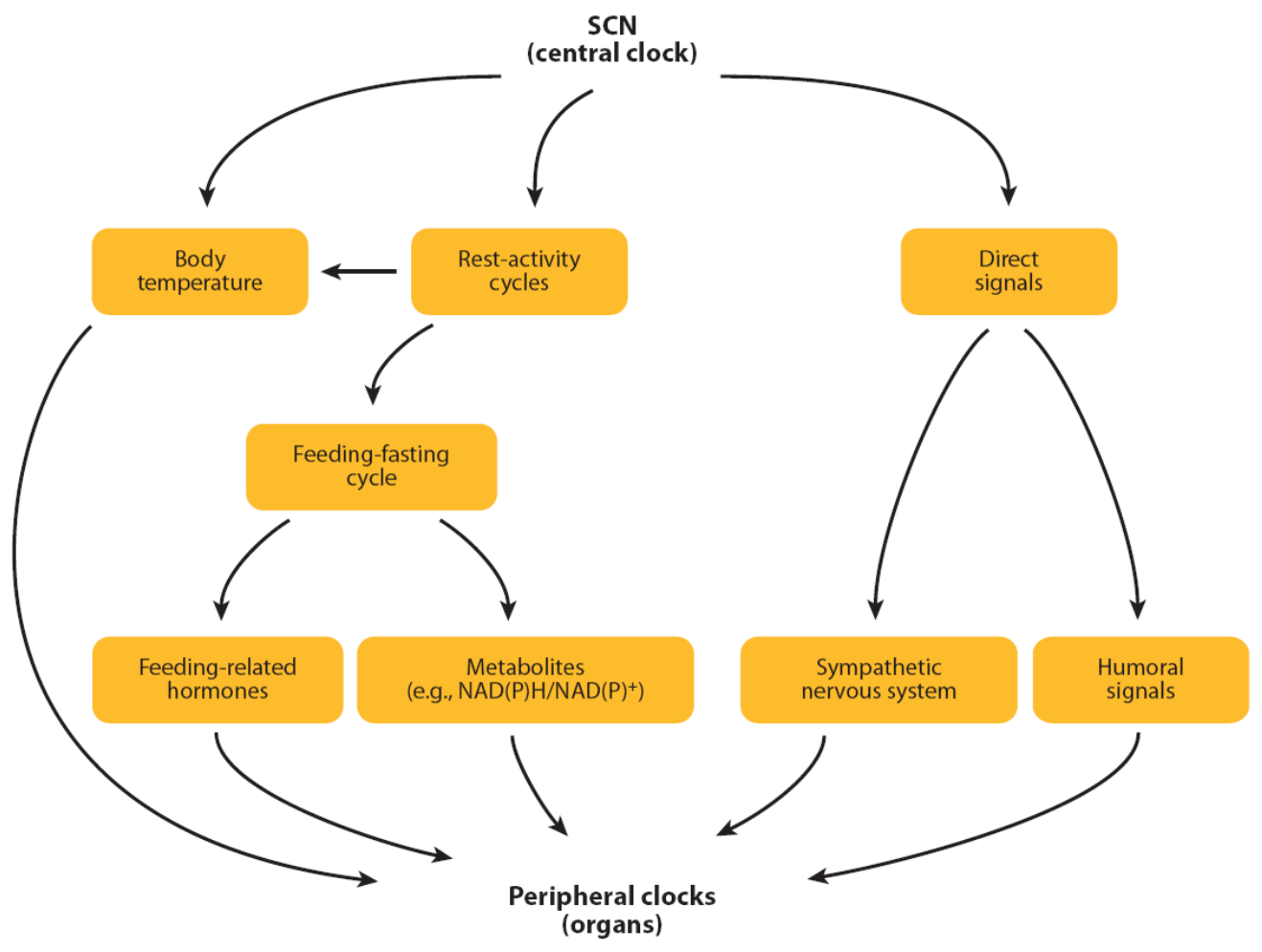

Figure 3. Peripheral clock entrainment pathways. From (Dibner et al., 2010).

Interestingly, an intricate balance between sympathetic and parasympathetic innervation seems to be important for maintaining rhythmic output in a peripheral tissue. Cailotto et al. found that complete hepatic denervation combined with a non-circadian feeding schedule did not abolish the 24-h profile in plasma glucose nor daily rhythms in the expression of key liver enzymes, but either sympathetic or parasympathetic denervation did so (Cailotto et al., 2008).

Whereas light is the main Zeitgeber signal for the central clock, peripheral clocks are more readily entrained by feeding cues. Indeed, U. Schibler and others convincingly demonstrated that feeding restricted to few hours during the light phase, when mice do not normally forage for food, can reset peripheral circadian oscillators without altering the central clock rhythms (Damiola et al., 2000; Stokkan et al., 2001). Feeding, however, can serve not only as a synchronizer of a local clock, but also induce rhythmic transcription, when applied in a rhythmic manner. Indeed, Vollmers et al. showed that restricted feeding is capable of inducing rhythmic gene expression in the liver of circadian clockdeficient Cryl/Cry2 animals (Vollmers et al., 2009). Interestingly, animals on restricted feeding behaviorally anticipate the time when food is available. This so-called food anticipatory activity does not require intact SCN structures nor known clock genes (Marchant and Mistlberger, 1997; Storch and Weitz, 2009), suggesting that an additional circadian oscillator of unknown location is likely to be involved (Mistlberger, 2011). Moreover, it seems that the central clock has mainly an inhibitory effect on food entrainment. For instance, SCN lesions were shown to augment food anticipatory activity in 
rats (Angeles-Castellanos et al., 2010). In line, elimination of neural signals from the SCN by surgical sympathectomy made the circadian clock in salivary glands more sensitive to feeding entrainment (Vujovic et al., 2008). The entrainment of peripheral clocks by metabolic cues can be achieved via different pathways (Asher and Schibler, 2011; Bass and Takahashi, 2010; Kovac et al., 2009). NADdependent enzymes (e.g., sirtuins and poly[ADP-ribose] polymerases), redox- and/or temperaturedependent transcription factors (e.g., CLOCK, NPAS2, and HSF1), nutrient-sensing transcriptional regulatory proteins (e.g., FOXO-p300, nuclear receptors, PGC-1) and protein kinases (e.g., AMPK), are plausible candidates for conveying a metabolic state of the cell to the core clock machinery (Asher and Schibler, 2011). As an example, the DNA binding activity of the CLOCK/BMAL1 transcriptional complex is dependent on the redox state of the cell (Rutter et al., 2001). The molecular candidates for mediating metabolic effects on the clock include NAD-dependent enzymes such as SIRT1 and PARP1 and the rate limiting enzyme of NAD synthesis, NAMPT (Asher and Schibler, 2011). However, systemic signals that mediate entrainment of peripheral clocks by feeding still remain obscure.

Finally, the SCN neural network is resistant to physiological fluctuations of body temperature, whereas the circadian rhythms in peripheral tissues can be entrained by temperature cycles, involving the heat shock pathway (Brown et al., 2002; Buhr et al., 2010; Saini et al., 2012). Therefore SCNmediated control of circadian rhythms in body temperature can serve as a universal cue for the entrainment of cell-autonomous oscillators throughout the body (Buhr et al., 2010).

\subsection{Circadian rhythms and disease: clinical applications}

A link between disruption of circadian rhythms and pathology is well documented. On the one hand, aberrant behavioral/social rhythms seen in shift workers, people experiencing the jet lag syndrome or suffering from chronic "social jet lag" may cause development of a range of physiological/metabolic abnormalities and facilitate disease susceptibility and progression. Indeed, repetitive jet lag was shown to produce cognitive deficits, temporal lobe atrophy $(\mathrm{Cho}, 2001)$ and promote tumor growth (Filipski et al., 2004). In addition, a significantly higher incidence of autoimmune diseases such as autoimmune hypothyroidism and rheumatoid arthritis has been reported in chronic shift workers (Magrini et al., 2006; Puttonen et al., 2010). Finally, Roenneberg and colleagues found that misalignment of endogenous and behavioral rhythms, i.e. social jet lag, of just one hour increases the chances to be overweight/obese by 30\% (Roenneberg et al., 2012; Roenneberg et al., 2013).

In line, genetic models of clock deficiency revealed that impaired circadian clock function leads to obesity (Shostak et al., 2013b; Turek et al., 2005), diabetes (Marcheva et al., 2010) and mood disorders such as mania (Hampp et al., 2008; Roybal et al., 2007). Interestingly, a lack of certain clock genes does not always result in impaired resistance to developing a disease. As an example, flies with 
the $\mathrm{per}^{01}$ mutation are more efficient in combating infection compared to wild-type controls (Lee and Edery, 2008).

In humans, mutations in clock genes lead to disorders associated with abnormal sleep-wake cycles (Landgraf et al., 2012). For example, patients with familiar advanced sleep phase syndrome (FASPS) often carry mutations in the Per2 and Csnkld genes (Toh et al., 2001; Xu et al., 2005).

On the other hand, diseases can disrupt the circadian clock. Circadian alterations have been described both in patients and mouse models with various disorders such as obesity (Kohsaka et al., 2007), some forms of cancer (Yang et al., 2006) and neurological disorders (Albrecht, 2013b), including Alzheimer's disease (Stranahan, 2012), schizophrenia (Oliver et al., 2012) and unipolar and bipolar depression (Boivin, 2000).

Additionally, some chronic diseases show diurnal variations in symptoms, as it is well known for rheumatoid arthritis (Gibbs and Ray, 2013). Such patients suffer from increased painful joint stiffness in the early morning, which correlates with an early morning rise in circulating levels of proinflammatory cytokines, such as interleukin (IL)-6.

Our fundamental knowledge on circadian rhythms of physiology and behavior helps establish a firm background for chronopharmacology and chronotherapy - time of day-dependent clinical interventions for more efficient treatment of disorders (Levi and Schibler, 2007; Musiek and Fitzgerald, 2013). Promising trends in this emerging field are circadian rhythm-based administration of anti-tumor drugs (Levi and Schibler, 2007; Ortiz-Tudela et al., 2013) and timed bone marrow transplantation (Scheiermann et al., 2012; Shi et al., 2013). As shown in two consecutive randomized multicenter trials involving a total of 278 patients with metastatic colorectal cancer, the chronomodulated regimen of chemotherapy achieved $51 \%$ objective tumor responses, compared with $30 \%$ in patients receiving constant rate infusion (Levi et al., 1997). In mice, when the bone marrow was transplanted in the beginning of the activity phase (i.e. at night), all recipients survived, which represents significantly better engraftment of hematopoietic progenitor/stem cells (Scheiermann et al., 2012). Strikingly, only $50 \%$ of mice survived after the transplantation during daytime.

\subsection{GCs, stress response, and the circadian clock}

Glucocorticoid (GC) hormones (mainly cortisol in humans, corticosterone (CORT) in rats and mice) play a key role in daily regulation of normal physiology and behavior as well as in orchestration of bodily responses to stress. Under homeostatic conditions GCs are produced by the adrenal glands in a rhythmic fashion. GCs are secreted in regular, hourly pulses and the amplitude of these ultradian pulses varies over the course of a day, reaching its peak in the beginning of the activity phase (early morning in diurnal animals, including humans, and early night in nocturnal animals). Upon acute 
stress a rapid surge of GC synthesis overrides ultradian and circadian rhythms, drives a stress response throughout the body, and allows an organism to shift physiology and behavior in order to adapt to stressful stimuli. However, chronic, persistent stress and over-production of GCs can lead to diverse pathologies.

The circadian clock and GCs are closely interconnected. Corticoid receptors can control expression of some clock genes and interact directly with components of the molecular clock. Therefore the effects of GCs in tissues are partially mediated via modulation of the circadian clockwork.

The importance of GCs for an organism is clearly seen in patients and experimental animals with impaired GC production. Rats that survived adrenalectomy (ADX) become extremely sensitive to various stressors under compromised conditions, even when maintained on saline solution to restore mineralocorticoid deficiency (Darlington et al., 1990). In humans, chronic hypocortisolism with normal aldosterone levels and electrolyte balance is featured in familial GC deficiency (FGD), a rare autosomal recessive disorder due to impaired adrenal sensitivity to adrenocorticotropin (Clark et al., 2009; Clark and Weber, 1998). Already in the neonatal period or early childhood, patients with FGD develop recurrent hypoglycemia and frequent, persistent infections that might be lethal if untreated.

\subsubsection{Anatomy and physiology of the adrenal gland}

GCs are produced in the adrenal gland by adrenocortical cells of the zona fasciculata. In addition, the adrenal gland produces other hormones essential for stress responses and the regulation of body homeostasis. Firstly, the mineralocorticoid aldosterone produced by zona glomerulosa cells is important for regulation of blood pressure and salt homeostasis (Thomas and Harvey, 2011). Under conditions of hypotension, aldosterone is released in response to angiotensin II and elevates blood pressure through changes in vascular tone and electrolyte transport across absorptive epithelia. Alternatively, aldosterone promotes the secretion of excess potassium from the body under conditions of hyperkalemia. Secondly, catecholamines (adrenalin and noradrenalin) released by medullary chromaffin cells upon activation of the sympatho-adrenomedullary system coordinate a complex of fast physiological responses to stress, known as the "fight-or-flight" reaction (reviewed in (Kvetnansky et al., 2009); section 1.5.8.). Finally, the adrenal cortex of humans and some nonhuman primates (but not rats and mice) contains androgen-producing cells that synthesize dehydroepiandrosterone (DHEA) and its sulfoconjugate DHEA-S during pre- and postnatal periods of development (Nguyen and Conley, 2008). 


\subsubsection{Regulation of GC production: Anatomy of the HPA axis}

Basal GC production and GC responses to stress are under control of upstream brain regions that, together with the adrenal gland, form the so-called hypothalamic-pituitary-adrenal (HPA) axis (Figure 4). It consists of the following molecular and anatomical components.

Hypothalamus. The first hub of the HPA axis - parvocellular neurons in the hypothalamic paraventricular nuclei (PVN) - integrates signals from various brain regions including those involved in processing homeostatic perturbations, pain and inflammation (brainstem, hypothalamic nuclei, circumventricular organ) as well as from limbic structures (amygdala, hippocampus, prefrontal cortex) that deal with more complex psychogenic and systemic stimuli (for a comprehensive review, see (Ulrich-Lai and Herman, 2009)). Upon activation, the PVN neurons secrete two peptides corticotrophin-releasing hormone/factor $(\mathrm{CRH})$ and arginine vasopressin (AVP) - into the hypophyseal portal blood.

Pituitary. CRH and AVP synergistically induce proopiomelanocortin (POMC) mRNA expression in corticotroph cells of the anterior pituitary via oligomerization of corresponding receptors CRHR1 and V1bR and activation of the adenylate cyclase-cAMP-protein kinase A (PKA) signaling cascade (Bonfiglio et al., 2011). The POMC polypeptide is cleaved by the prohormone convertase 1 (PC1) forming a major stimulator of GC synthesis - adrenocorticotropic hormone (ACTH) (Bicknell, 2008). ACTH is processed and stored in acidified secretory granules and released via exocytosis upon demand (Moore et al., 2002).

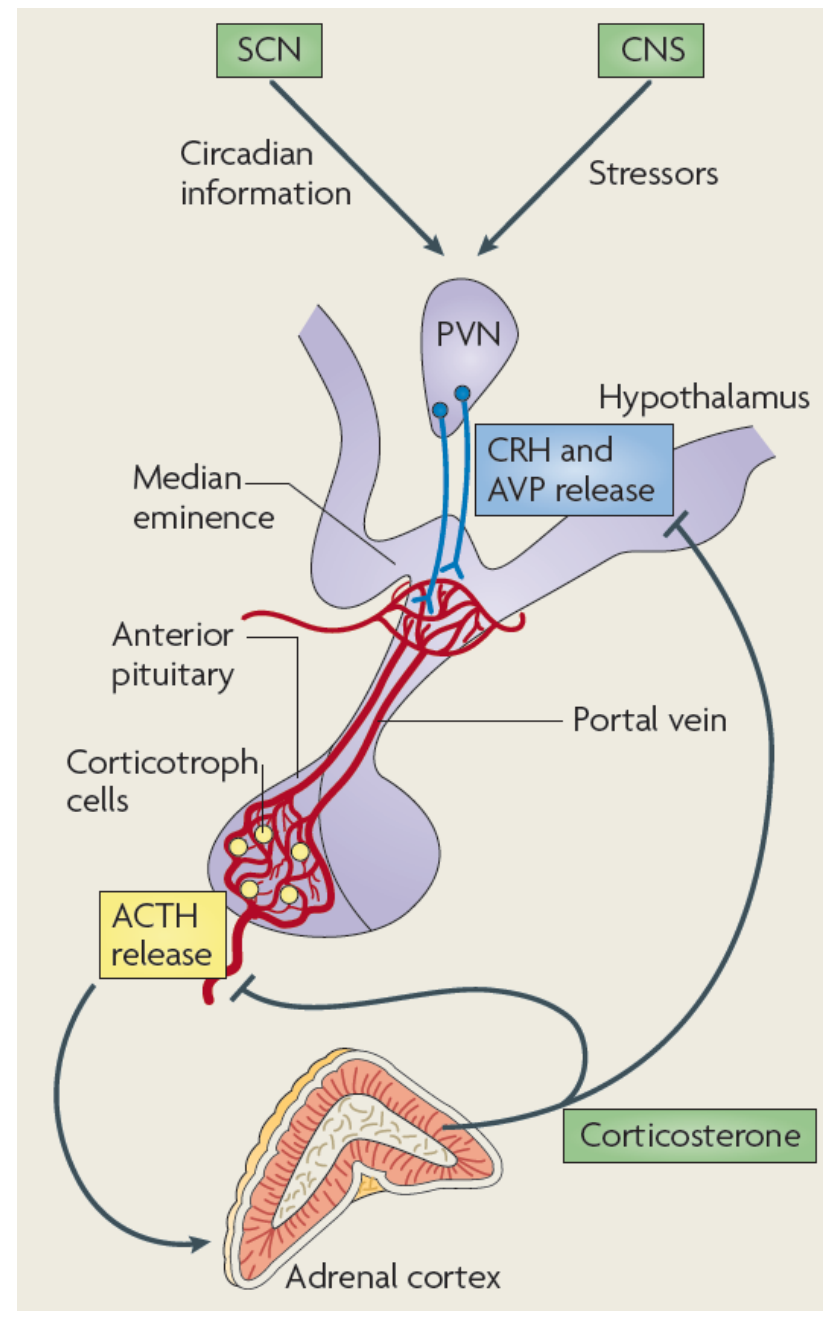

Figure 4. A schematic representation of the HPA axis. From (Lightman and Conway-Campbell, 2010).

CNS—central nervous system. 
Adrenal cortex. ACTH released into the bloodstream binds to the melanocortin-2 receptor (MC2R) in adrenocortical cells and acutely induces streroidogenesis. Similar to CRHR1, MC2R is a transmembrane G-protein coupled receptor that activates cAMP-PKA signaling. Within minutes, ACTH up-regulates mobilization of lipid droplet-stored cholesterol esters and steroidogenic acute regulatory protein (StAR)-mediated increase of cholesterol delivery to the CYP11A1 (P450scc) enzyme in the inner mitochondrial membrane (Hu et al., 2010; Stocco, 2001). StAR-mediated delivery of cholesterol to the substrate site of P450scc for conversion to pregnenolone represents a key ratelimiting step of steroidogenesis (Stocco, 2001). Prolonged stimulation of adrenocortical cells with ACTH increases transcription of other steroidogenic enzymes (CYP17, CYP21A1, CYP11B1) and elevates cholesterol uptake via LDL receptor-mediated and SR-BI-mediated pathways (Hu et al., 2010; Simpson and Waterman, 1988). In addition, HMG-CoA reductase, a rate-limiting enzyme of de novo cholesterol synthesis, is also rapidly stimulated upon exposure of adrenal cells to ACTH (Hu et al., 2010).

Activity of the HPA axis is influenced by a number of other hormones and cytokines. For instance, oxytocin, opioid peptides and endocannabinoids suppress ACTH secretion via different mechanisms, while VIP, catecholamines, ghrelin, and the IL-1 and IL-6 act in a stimulatory manner (Papadimitriou and Priftis, 2009).

\subsubsection{Regulation of GC production: Autonomic innervation}

In addition to hormonal control of adrenal function, there is also extensive innervation from sympathetic and sensory circuits. Both of these are carried in the splanchnic nerves (Ulrich-Lai and Engeland, 2000). Medullary chromaffin cells are per se modified postganglionic sympathetic neurons that are controlled by preganglionic sympathetic fibers. Nerve supply to the adrenal cortex is more complex. Adrenocortical cells are innervated by sympathetic postganglionic and primary afferent nerve fibers, but there is little evidence for direct parasympathetic innervation (Ehrhart-Bornstein et al., 1998; Engeland, 2013). In addition, cortical cells are intertwined anatomically with chromaffin cells and the latter release a broad spectrum of neuropeptides (VIP, NPY, CGRP, substance P, catecholamines and acetylcholine); for many of these peptides there are receptors on adrenocortical cells (reviewed in (Ehrhart-Bornstein et al., 1998)). These cortico-medullary interactions occur via intra-adrenal nerve fibers that originate in the medulla and innervate the outer layers of the cortex.

Autonomic innervation of the adrenal gland contributes to regulation of adrenal sensitivity to ACTH (reviewed in (Engeland and Arnhold, 2005)). Indeed, splanchnic nerve transsection in rats or lambs leads to reduced GC production and ACTH sensitivity in the evening (Dijkstra et al., 1996a; Edwards et al., 1986; Ulrich-Lai et al., 2006). On the other hand, electrical stimulation of the splanchnic nerve in various species significantly increases adrenal GC production (Bornstein et al., 1990; Edwards and Jones, 1987; Engeland and Gann, 1989). It remains still unclear how sympathetic nerves alter adrenal 
sensitivity to ACTH, but various potential scenarios were proposed (Engeland, 2013). One possibility is based on modulation of ACTH delivery via changes in cortical blood flow. According to another hypothesis, the release of catecholamines from chromaffin cells could stimulate ACTH receptor signaling via $\beta$-adrenergic receptor-mediated changes in intracellular cAMP concentrations.

Extra-adrenal GC production. In addition to adrenocortical cells other tissues, including thymus, intestine, skin and brain, express steroidogenic enzymes and regulatory proteins and thus are principally capable of producing GCs (Talaber et al., 2013; Taves et al., 2011). Interestingly, local production of other components of the HPA axis such as CRH and ACTH was identified in the skin. Normally extra-adrenal GCs are synthesized in much lower concentrations compared to the adrenal gland and act in a paracrine, rather than endocrine, manner. However, it seems that under some specific conditions local GC synthesis might be boosted up to endocrine levels. P. Chambon's group recently reported that antibiotic-induced depletion of microbiota in ADX mice led to increased steroidogenesis in the intestine and a remarkable surge of plasma CORT concentrations reaching those observed in sham-operated animals (Mukherji et al., 2013).

\subsubsection{Regulation of GC sensitivity: from release to action}

After release into the bloodstream, most GCs bind to carrier proteins and can exert their effects almost on every tissue due to a wide distribution of corticosteroid receptors. Importantly, the final biological effects of GCs are determined not only by activity of GC synthesis, but also by sensitivity of target tissues to GCs (reviewed in (Quax et al., 2013)). Tissue responsiveness to GCs depends on many factors including those that regulate GC bioavailability, corticosteroid receptor signaling and transcriptional activity of the receptors.

Plasma GCs are continuously degraded. The half-life of GCs in mouse blood is short - less than 10 min (Conway-Campbell et al., 2010), but extends to 80-120 min in humans (Agarwal and Mirshahi, 1999). In the liver, GCs are reduced to dihydro- and tetrahydro-derivatives, following conjugation with glucuronic acid in both the hepatic and renal parenchyma, and excretion with urine and feces (Agarwal and Mirshahi, 1999; Girard et al., 2003).

Only about 5\% of plasma cortisol or CORT circulates in a non-protein-bound or "free" state, whereas the rest is bound to two carrier proteins produced by liver - corticosteroid-binding globulin (CBG, also known as transcortin and serpin A6; binds approximately $80 \%$ of plasma GCs) and albumin (Cizza and Rother, 2012; Henley and Lightman, 2011). Of note, GC dissociation from CBG is exquisitely sensitive to changes in body temperature and increases significantly in response to fever, thus facilitating anti-inflammatory effects of GCs in febrile patients (Lightman and Conway-Campbell, 2010). 
GCs bind to two kinds of corticosteroid receptors - mineralocorticoid (MR) and glucocorticoid (MR) receptors - and modulate gene expression via genomic and non-genomic signaling pathways. It is estimated that a large portion (1-20\%) of the mammalian genome can be positively or negatively regulated by GCs, depending of cell type and experimental settings (John et al., 2008; Lamia et al., 2011). Although the MR and GR share almost identical DNA binding domains, they show only limited (30 \%) gene overlap (Datson et al., 2001; Groeneweg et al., 2011). Therefore, both receptors can exert distinct cellular actions.

Although the ligand for both receptors is the same, the GR binds GCs with a 10-fold lower affinity, compared to the MR (de Kloet et al., 2005). The affinity of MR is sufficiently high to keep the receptor active even when GC production is low. By contrast, the low-affinity GR is occupied only at peaks of pulsatile GC secretion (section 1.5.5.) and during stress response (section 1.5.8.). The MR remains bound to the ligand for a long time (the half-life is $\approx 45 \mathrm{~min}$ ), while GR rapidly associates and dissociates from its endogenous ligand (the half-life is $\approx 5 \mathrm{~min}$ ). Of note, this is not the case with synthetic GC agonists such as dexamethasone (Dex) (Sarabdjitsingh et al., 2012; Stavreva et al., 2009).

Compared to the GR, which is almost ubiquitously expressed, the pattern of MR expression is limited to some specific tissues, mainly the polarized tight epithelia of the kidney where it mediates aldosterone-dependent transepithelial sodium transport. MR is also found in epithelial cells of lung, eyes and skin, in cardiomyocytes, macrophages, adipocytes, the hypothalamus and the hippocampus.

Local concentrations of GCs in tissues are controlled by two $11 \beta$-hydroxysteroid dehydrogenase isoenzymes. 11 $\beta$-HSD type 1 , which is expressed, for example, in the liver and adipose tissue, converts biologically inactive cortisone into active cortisol, whereas 11ßHSD type 2 has the opposite effect, particularly in the kidney where it prevents GC-mediated activation of the high-affinity MR (Chapman et al., 2013; Funder et al., 1988). In addition, the multidrug resistance P-glycoprotein (MDR1) lowers availability of GCs via active transport of the hormones out of the cell, affecting GC transport to the brain (Karssen et al., 2001; Mason et al., 2008; Meijer et al., 1998; Tsujimura et al., 2008).

It has been reported that GR activity and transcriptional specificity are modulated by alternative splicing of the $N r 3 c 1$ gene, alternative translation of the GR protein, and posttranslational modifications (phosphorylation, ubiquitination, sumoylation). Next, folding, affinity and intracellular and/or nuclear transport of GR are dynamically regulated by ligand binding. In the absence of a ligand, GR is located in the cytoplasm as part of a multiprotein complex composed of several heat shock proteins and other co-chaperones. The composition of the GR-chaperone complex is plastic and defines GR affinity and its sensitivity to proteolysis. 
Upon ligand binding GR-GC complexes form dimers, translocate to the nucleus and bind to GCresponse elements (GREs) in regulatory regions of many genes. This can promote the recruitment of several coactivators and chromatin remodeling proteins and initiation of transcription. Alternatively, GCs can inhibit gene expression through protein-protein interactions with co-regulatory proteins and promoter-bound transcription factors such as AP-1 and NF- $\mathrm{kB}$ (so called "tethering"), direct association with inhibitory nGREs (Surjit et al., 2011), or recruitment of the corepressor GRIP1. However, the transcriptional outcome (activation versus suppression) of GC action is not defined exclusively by the presence or absence of the mentioned cis- and trans-regulatory elements, implying that additional epigenetic regulators are involved (Uhlenhaut et al., 2013). In addition to regulation of gene expression, GCs also have multiple rapid (within 5-15 min) effects that seem to be independent of transcription/translation and even resistant to blocking of the corticosteroid receptors (reviewed in (Groeneweg et al., 2011)). For instance, it was reported that ACTH secretion in pituitary can be rapidly inhibited by steroid administration in rats and the fast negative feedback is preserved in rats pretreated with a GR antagonist or transcription inhibitor (Hinz and Hirschelmann, 2000). On the other hand, some of the non-genomic effects can be mediated via membrane-bound forms of the GR or MR, as has been shown for rapid GC-mediated modulation of electrophysiological properties of hippocampal neurons ((Groeneweg et al., 2011); section 1.5.8.).

\subsubsection{Dynamic regulation of basal GC production}

Three main dynamic modes of GC production are seen in basal, non-stressed conditions - ultradian, circadian, and seasonal.

\section{Ultradian GC rhythms}

In rats and humans, activity of the HPA axis including plasma ACTH and GC levels as well as GC concentrations in target organs follow an ultradian, hourly pattern (Henley et al., 2009a; Lightman and Conway-Campbell, 2010; Sarabdjitsingh et al., 2012). Ultradian GC rhythmicity seems to be independent of the circadian clock, being preserved in SCN lesioned animals (Waite et al., 2012).

Experimental studies and mathematical modeling suggest that ultradian GC pulsatility is unlikely to be generated by a hypothalamic pulse generator, but merely stems from a rhythmic, delayed feedback of GCs on pituitary (Lightman and Conway-Campbell, 2010; Walker et al., 2010). Indeed, Engler and co-workers showed that disconnection of the hypothalamus from the pituitary in sheep does not impair pituitary-adrenal ultradian activity (Engler et al., 1990).

Responsiveness of the HPA axis to stress varies at different phases of the ultradian GC cycle. The rats displayed a considerably greater sensitivity of HPA axis when a 10-min noise stress was applied during the rising phase of a CORT pulse, while CORT responses during the falling phase were 
significantly diminished (Windle et al., 1998). Similarly, when the same dose of CORT was given by constant infusion rather than in a pulsatile pattern, the ACTH response to stress was markedly reduced (Sarabdjitsingh et al., 2010). This suggests that deregulated GC pulsatility may have a significant effect on the ability to respond properly to stress. Indeed, deregulation of the pulsatile pattern of GC secretion is associated with various disease states, including depression, other stress-related diseases, and obstructive sleep apnoea (Henley et al., 2009b).

Studies of ultradian GC responses in the AtT-20 corticotroph cell line revealed that each pulse of CORT initiates rapid and transient activation of intracellular GR and cyclical GR association with GREs in the promoter regions of the GC-induced genes, such as Per1 or serum-GC-regulated kinase 1 (Stavreva et al., 2009). Interestingly, after withdrawal of the ligand, GR stays in the nucleus and is reactivated by the following CORT pulse, resulting in re-association of GR with the chromatin template (Stavreva et al., 2009). Importantly, altering the pattern of GC delivery (pulsatile versus constant) results in different patterns of gene regulation.

\section{Circadian GC rhythms}

All main molecular constituents of the HPA axis, including CRH, ACTH and CORT/cortisol, follow a circadian pattern of production (Dickmeis, 2009). Several pathways contribute to generation of circadian GC rhythms: SCN-dependent rhythmic release of ACTH, rhythmic activity of the ANS, and a circadian clock in the adrenal gland (Figure 5).

\section{The central clock and the HPA axis}

Lesion experiments have convincingly shown that the SCN itself and intact SCN projections are essential for maintaining GC rhythms (reviewed in (Dickmeis, 2009; Kalsbeek et al., 2012)), since SCN grafts transplanted into SCN-lesioned hamsters are not sufficient to rescue the hormonal oscillations (Meyer-Bernstein et al., 1999). Firstly, the SCN sends indirect projections to the PVN neurons, mainly via subPVZ and DMH, to exert rhythmic AVP-dependent suppression of HPA axis activity (Figure 5). Work done by Buijs, Kalsbeek and colleagues clearly demonstrated that SCN ablation potentiates CORT release, while infusion of AVP into the PVN/DMH region inhibits it (Buijs et al., 1993; Kalsbeek et al., 1992; Kalsbeek et al., 1996a). A stimulatory effect of the SCN on GC synthesis was also reported (Cascio et al., 1987; Kalsbeek et al., 1996b), but its molecular nature is still unclear. 
Figure 5. Pathways involved in circadian regulation of GC production. From (Dickmeis, 2009).

DMH—dorsomedial hypothalamus, subPVZ — subparaventricular zone, mpPVN_medioparvocellular division of the PVN; I-interneuron of the PVN; $\mathrm{C}$ - corticotrope cell of the pituitary; IML_-intermediolateral column of the spinal cord.

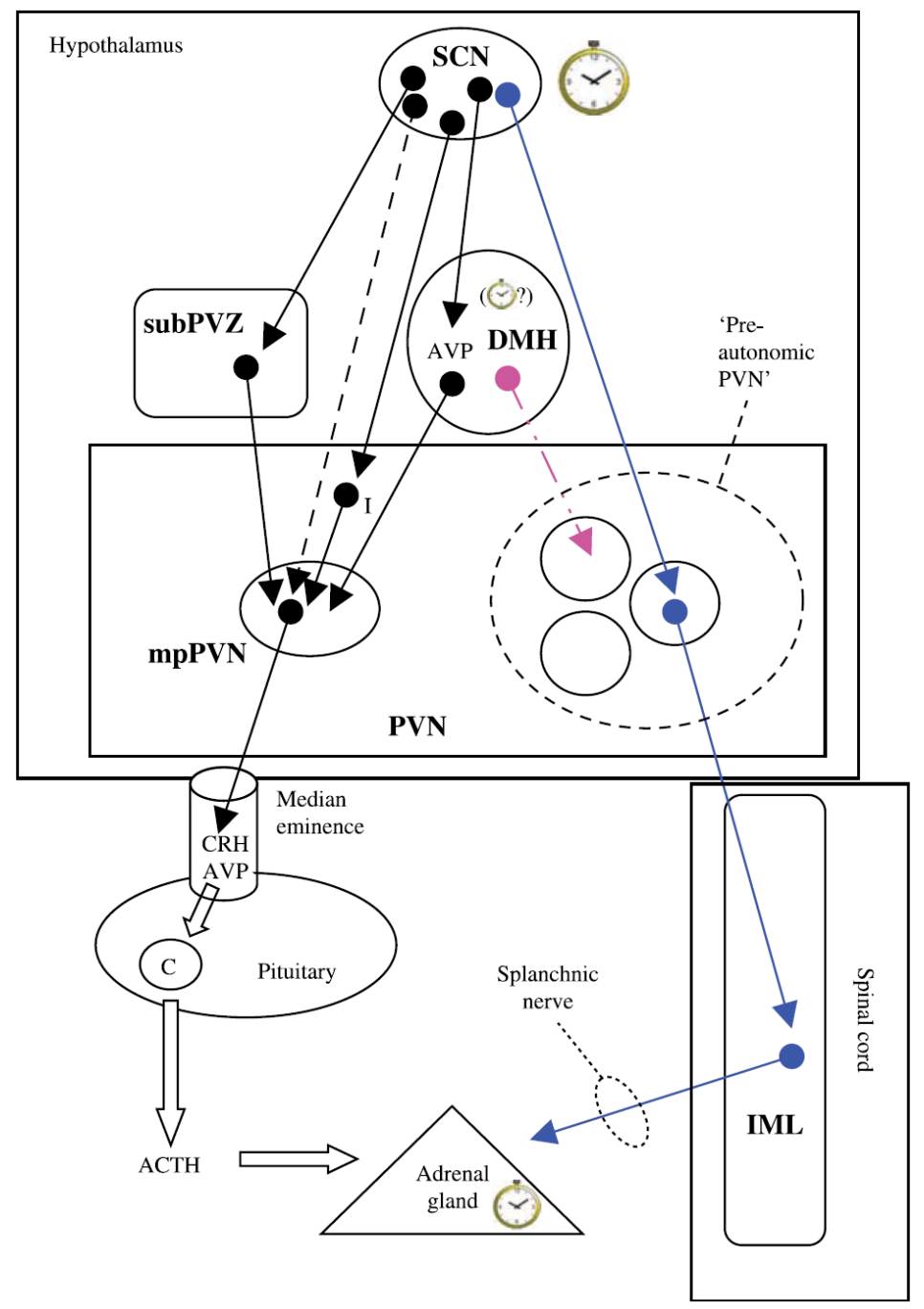

Circadian oscillators in other anatomical nodes of the HPA axis such as PVN ((Abe et al., 2002; Girotti et al., 2009; Guilding and Piggins, 2007) and refs within) and anterior pituitary (Abe et al., 2002; Bur et al., 2010; Chu et al., 2013; Girotti et al., 2009; Yoo et al., 2004) might also contribute to rhythmic GC production.

\section{The central clock and the ANS}

SCN neurons regulate rhythmic functioning of the adrenal gland through the autonomic nervous system that explains many physiological examples of dissociation between ACTH and GC production (reviewed in (Bornstein et al., 2008)). Indeed, hypophysectomy does not ablate GC rhythms, but does so only in combination with adrenal denervation (Meier, 1976; Ottenweller and Meier, 1982). Similarly, diurnal variation in adrenal steroidogenesis persists in CRH knockout mice with constant infusion of CRH (Muglia et al., 1997). Furthermore, 12-hour rhythmic cortisol release in behaviorally split hamsters, which presumably reflects asymmetric activity of left and right SCN (de la Iglesia et al., 2000), does not rely on rhythmic release of ACTH (Lilley et al., 2012). The multisynaptic SCN- 
adrenal pathway consists of pre-autonomic PVN neurons, sympathetic preganglionic IML neurons of the spinal cord, and finally the splanchnic nerve that innervates the adrenal gland (Buijs et al., 1999; Dickmeis, 2009).

Seminal work by Okamura and co-workers demonstrated that the autonomic innervation of the adrenal gland is required for light-induced GC responses. Ishida et al. showed that a 30-min exposure to light is able to up-regulate Per gene expression in the adrenal gland and induce GC release independent of ACTH via the SCN-ANS pathway (Ishida et al., 2005). Interestingly, it seems that epinephrinemediated medulla-cortex signaling is required for this effect. This is consistent with observations that adrenal demedullation prevents splanchnic nerve-mediated GC release (Ulrich-Lai et al., 2006). Another functional aspect of autonomic innervations is that the SCN-adrenal neural connections are important for maintaining circadian regulation of adrenal sensitivity to ACTH in vivo, since SCN ablation or adrenal denervation severely compromise circadian variation in adrenal ACTH responsiveness ((Dallman et al., 1978; Dijkstra et al., 1996b; Kaneko et al., 1980; Kaneko et al., 1981; Sage et al., 2002; Ulrich-Lai et al., 2006); reviewed in (Engeland and Arnhold, 2005)).

Light is not the only signal relevant for GC rhythms. Daily feeding patterns are able to adjust the circadian phase of peripheral clocks, e.g. in liver (Damiola et al., 2000; Stokkan et al., 2001), and of daily GC oscillations, independently of the SCN (Krieger et al., 1977). Interestingly, daytime restricted feeding strongly dampens the circadian clock in the pituitary, even upon adrenalectomy (Bur et al., 2010; Girotti et al., 2009), which may point towards ANS-mediated control of the restricted feeding-induced GC rhythm.

\section{The adrenal clock}

Several lines of evidence support the view that both adrenocortical and chromaffin cells contain functional circadian clocks.

It is well documented that the expression of clock genes (Arntl, Npas2, Cry1, Per2, Per3, Nrld1) and first-order clock-controlled genes ( $D b p, T e f)$ in the adrenal gland follows a 24-hour rhythm (Bittman et al., 2003; Fahrenkrug et al., 2008; Lemos et al., 2006; Oster et al., 2006a; Oster et al., 2006b; Son et al., 2008; Torres-Farfan et al., 2006; Valenzuela et al., 2008). Importantly, clock gene rhythmicity is sustained in cultured adrenal explants from Per $2:: l u c$ and Bmall::Eluc circadian reporter mice (Noguchi et al., 2012) (Leliavki and Oster, unpublished). Furthermore, Oster et al. showed that approximately $5 \%$ of the adrenal transcriptome (more than 1,500 genes) is expressed in a circadian fashion (Oster et al., 2006a; Oster et al., 2006b) (Figure 6). Among rhythmic genes are those involved in cholesterol uptake (Ldlr, Scarb2) and transport (Slc25a, Bzrp), steroid biosynthesis and its regulation (Hsd17b7, Lss, Stard4, Star, Sp1, Por), ACTH receptor signaling (Mc2r, Acdy5, protein kinase A, protein phosphatase 1 subunits) as well as catecholamine metabolism (Maoa, Nr4a2, Pnmt). 
It has been reported that rhythmic expression of StAR, a key rate-limiting cholesterol transporter, is under E-box-dependent circadian regulation by CLOCK and BMAL1 (Kil et al., 2012; Son et al., 2008; Ulrich-Lai et al., 2006). In addition, a circadian microarray study revealed that a significant portion of the found cycling genes represents some general biological pathways such as protein catabolism and nucleosome assembly (Oster et al., 2006a). Interestingly, rhythmic steroidogenic genes peak at day-to-night transition, when GC synthesis is maximal in mice, matching the phase of typical E-box-regulated clock-controlled genes. On the other hand, the expression of nucleosome assembly genes has a reversed circadian pattern, reaching its maximum around early morning.

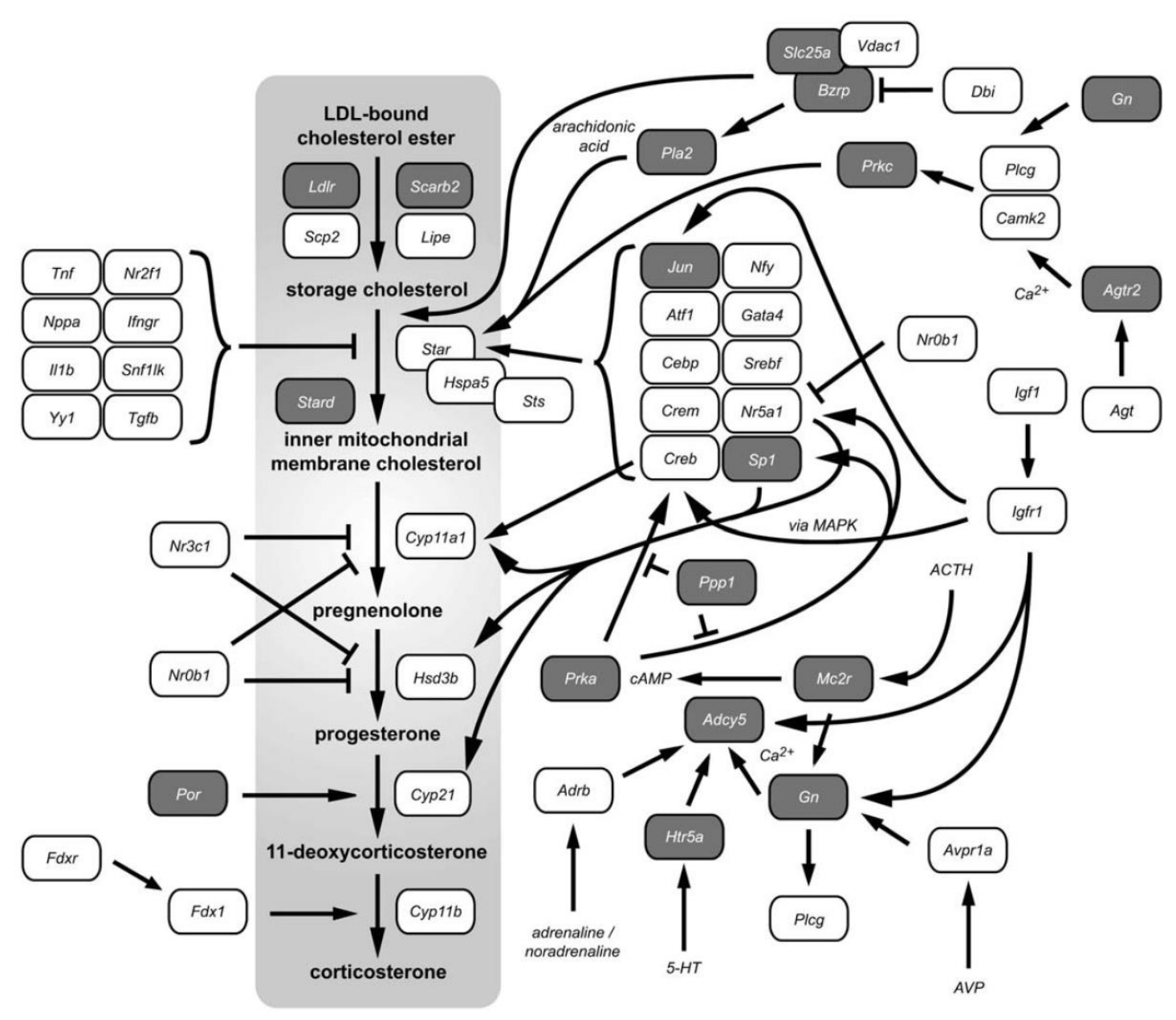

Figure 6. Circadian clock-regulated genes involved in the control of adrenal GC biosynthesis. Gray boxes indicate rhythmically expressed genes. From (Oster et al., 2006b).

24-hour oscillations of adrenal function were reported in vivo and found to be preserved in culture. In 1964, Andrews and Falk demonstrated that cultured hamster adrenal glands maintain circadian patterns in respiration and steroid secretion ex vivo (Andrews and Folk, 1964). Similarly, rhythmic GC synthesis persists in cultured adrenocortical Y-1 cells (Son et al., 2008). Recently, Kil and co-authors 
provide evidence that rhythmic activity of the peroxiredoxin system is critical for removal of hydrogen peroxide generated as a side-product of CYP11B1-mediated CORT synthesis (Kil et al., 2012). This prevents oxidative damage of adrenocortical cells during the peak of GC production. Furthermore, it was demonstrated the adrenal clock autonomically controls circadian variation of ACTH responsiveness in cultured adrenal explants (Oster et al., 2006b). In accordance to this, in vitro knockdown of Cry2 in capuchin monkey adrenal glands decreases cortisol production in response to ACTH (Torres-Farfan et al., 2006). The CRY2 down-regulation leads to dramatic reduction of the clock protein BMAL1 and two steroidogenic factors - StAR and 3 $\beta$-HSD. This suggests that the adrenal clock may serve as a critical hub in the SCN-ANS-mediated regulation of circadian rhythms in ACTH sensitivity (Dickmeis, 2009).

To address a role of the adrenal clock in generation of circadian GC rhythms in vivo, Oster and colleagues transplanted Per $2 / \mathrm{Cryl}^{-/-}$adrenal grafts under the kidney capsule of adrenalectomized wild-type mice, and vice versa (Oster et al., 2006b). This approach revealed that the intact adrenal clock is not sufficient to sustain GC rhythms under constant conditions, but the light-dark (LD) cycle still can drive diurnal changes in GC production, though with a reduced amplitude. On the other hand, adrenal clock deficiency led to a dampened rhythm of CORT production, both in LD and constant dark (DD) conditions. Alternatively, Son and co-workers performed genetic ablation of the adrenal clock (Son et al., 2008). They generated a transgenic mouse in which an antisense Bmall gene coding sequence was expressed under control of the ACTH receptor $(M c 2 r)$ promoter. In partial agreement with the previous finding, daily rhythms of adrenal CORT were preserved in the mutant mice kept in LD, but disappeared in constant conditions. Taken together, these observations suggest that the adrenal clock is required for maintaining high amplitude circadian GC rhythms.

\section{Seasonal GC rhythms}

GC production in many vertebrates varies over the course of the year (Romero, 2002), normally peaking during a breeding period. Several hypotheses have been proposed to explain the nature of annual GC fluctuations. It is plausible that these changes may be driven by a need to mobilize energy or adjust behavior for coping with stress changes. On the other hand, annual GC rhythms may represent an evolutionary consequence of seasonal differences in the risks of being exposed to stressors (Romero, 2002). Importantly, the existence of a circannual clock in the pituitary of sheep has recently been proposed (Lincoln et al., 2006), but its potential contribution to seasonal GC variation remains obscure.

\subsubsection{Circadian GC rhythms and synchronization of tissue clocks}

Schibler and co-workers were the first to demonstrate that GCs can reset the circadian clock, both in vitro (Balsalobre et al., 1998) and in vivo (Balsalobre et al., 2000). Intriguingly, despite the fact that 
the GR is present in most peripheral tissues and brain areas, the SCN neurons are devoid of the receptor (Balsalobre et al., 2000), shielding the master clock from direct GC feedback. This makes GCs ideal candidates for unidirectional hormonal mediators that link the SCN clock and circadian clocks in the periphery.

GCs can reset the circadian clock machinery via different routes. One mechanism is based on transcriptional regulation of clock genes. Indeed, functional GREs are present in regulatory regions of several clock genes, including Per1 and Per2, E4bp4, Npas2 and some others (So et al., 2009; Yamamoto et al., 2005). Another mechanism involves direct/indirect protein-protein interactions and posttranslational modifications. CRY proteins inhibit GR activity and transcription of a large portion of GC-responsive genes via direct interaction with the GR (Lamia et al., 2011). Further, Cheon et al. found that GR-BMAL1 interaction is required for GC-mediated Per2 induction and the delayed circadian phase in mouse fibroblasts (Cheon et al., 2013a). Similarly, the CLOCK/BMAL1 complex can physically interact with the ligand-binding domain of the GR to suppress GC-induced transcriptional activity (Nader et al., 2009). CLOCK inherits histone acetyltransferase activity (Doi et al., 2006) and acetylates a lysine cluster in a hinge region of the human GR, thus reducing its affinity to GREs (Charmandari et al., 2011; Nader et al., 2009). GR is rhythmically expressed in many tissues, for example, in the rat liver and human peripheral leukocytes (Xu et al., 1991), implying that complex bi-directional communication between the GR and the circadian clock modulates the final output of rhythmic GC action in tissues.

Another example of such bidirectional regulation of rhythmic GC sensitivity is at the level of GC transport in blood. CBG concentration in blood and expression of its transcript in liver shows diurnal variation with a rhythmicity pattern parallel to that observed for GCs ((Hsu and Kuhn, 1988; Hughes et al., 2009) and refs within). On the other side, CBG rhythms per se are regulated by GCs, since adrenalectomy in rats abolishes its diurnal variation (Hsu and Kuhn, 1988).

Rhythmic transcription of many genes relies on circadian GC oscillations. Oishi et al. showed that adrenalectomy in mice causes around $60 \%$ reduction in the number of rhythmic genes in liver, supporting a view that adrenal gland/GCs play a role in generation of circadian rhythms in GC target tissues (Oishi et al., 2005). In line, Bmall gene knock-down in adrenocortical cells led to significant dampening of Perl gene expression rhythms in the liver, kidney and pancreas under constant conditions (Son et al., 2008). Furthermore, GCs might be important in synchronization of brain clocks outside the SCN. For instance, in mice rhythmic PER2 protein expression in the central amygdala is dependent on GC-GR signaling (Segall et al., 2009). However, it remains unclear how direct and circadian clock-mediated GC effects are integrated in circadian control of global transcription.

Finally, it has been demonstrated that circadian GC rhythms and the adrenal clock play an important role in adjusting the speed of behavioral re-entrainment and synchronized resetting of peripheral 
clocks to a new light-dark cycle during jet lag. Phase shifts of locomotor activity rhythms happen much faster in mice transplanted with circadian clock-deficient adrenals and can be changed through pharmacological manipulation with the phasing of GC production (Kiessling et al., 2010). These findings suggest that GCs can influence the activity of the central clock and behavioral rhythms via an indirect pathway. In line with this, Le Minh and colleagues showed that phase shifts in peripheral oscillators, such as liver and kidney, induced by day-time feeding happen significantly faster in adrenalectomized mice and are GR-dependent (Le Minh et al., 2001). Therefore, GCs play an essential role in communication between central and peripheral clocks during responses to different entraining signals such as light-dark and feeding cycles.

\subsubsection{Circadian GC oscillations and rhythmic regulation of tissue functions}

Either by modulation of circadian clock activity or direct transcriptional regulation of various GC target genes, daily GC rhythms were shown to be important for GC-dependent rhythmic control of many physiological processes in the mammalian body, including learning and memory, mobilization of energy stores, tissue homeostasis and immune functions.

The Born group and others provided evidence that changes in plasma cortisol levels during sleep in humans are important for memory consolidation (reviewed in (Wagner and Born, 2008)). The first half of the night, when cortisol levels are low, is dominated by slow wave sleep (SWS), whereas rapid eye movement (REM) sleep prevails in the second half, when the peak of cortisol production occurs. An increase of plasma GC concentrations during SWS-rich early sleep counteracts hippocampusdependent declarative memory consolidation (Plihal and Born, 1999). On the other hand, preventing the elevation of cortisol during late REM sleep-rich sleep by administration of metyrapone impairs hippocampus-dependent declarative memory, but enhances amygdala-dependent emotional aspects of memory (Wagner et al., 2005). In line with this, Liston et al. showed that circadian GC oscillations in mice are important for formation and stabilization of new synapses and hence facilitate long-term memory retention (Liston et al., 2013). To the contrary, chronic and excessive GC exposure eliminates learning-associated new postsynaptic spines and disrupts previously acquired memories. Interestingly, GCs promote rapid spine formation through a non-transcriptional mechanism recruiting the LIM kinase-cofilin pathway and increase spine elimination through transcriptional mechanisms involving MR activation (Liston et al., 2013).

It still remains unclear to which extent basal circadian rhythms of GCs contribute to daily changes in glucose, lipid and protein metabolism. For instance, adrenalectomy does not affect rhythmic changes in hepatic glycogen in ad libitum fed rats (Bouillon and Berdanier, 1981). On the other hand, the diurnal rhythm of plasma leptin is significantly attenuated in ADX rats supplemented with a CORT pellet (Nishiyama et al., 2000). Two key lipolytic enzymes, hormone-sensitive lipase (HSL) and adipose triglyceride lipase (ATGL) found to be under control of the circadian clock in adipose tissue 
(Shostak et al., 2013a) are also directly regulated by GCs (Xu et al., 2009). This assumes an intricate interaction between local and systemic components of the circadian timing system in adipose tissues.

Daily rhythmic changes in the immune system and immune responsiveness to antigen challenge also seem to be dependent on circadian GC rhythms. For instance, circadian variations of CD4+ and CD8+ $\mathrm{T}$ cell subpopulations in human blood are differentially regulated by release of cortisol (Dimitrov et al., 2009). Moreover, time of day-dependent variation in IgE-mediated allergic reaction in the skin is not observed in adrenalectomized mice as well as in Per2 mutant mice that display an aberrant daily GC production (Nakamura et al., 2011).

Finally, physiological GC oscillations contribute to the regulation of cell proliferation and tissue homeostasis. Kollet et al. recently showed that circadian GC rhythms in mice control proliferation of hematopoietic stem/progenitor cells and CXCL12 expression by bone marrow stromal progenitors (Kollet et al., 2013). Some data also suggest that circadian GC oscillations play a role in daily variation of neurogenesis in the dentate gyrus, cell proliferation in gastrointestinal mucosa and skin (reviewed in (Dickmeis and Foulkes, 2011)), as well as in circadian cell cycle rhythms in zebrafish (Dickmeis et al., 2007).

\subsubsection{Dynamics: regulation of GC response to acute stress}

\section{Stress response: from adaptation to pathology}

Information about various disturbances, actual or potential, in the environment of an individual are recognized and conveyed to specific brain areas. This causes a coordinated release of molecules, so called "stress mediators", that are responsible for coordination of different aspects of stress response (Joels and Baram, 2009). In addition to GCs, these stress mediators include monoamines (noradrenaline, serotonin and dopamine) and various neuropeptides (CRH, AVP, urocortins, orexin, dynorphin, oxytocin, NPY, ghrelin, and others). Such diversity is needed to cope with the entire range of stress-induced challenges, from conferring immediate attention and decision making to ensure survival at the moment of stress exposure, to storage of information about the stressful situation for better coping with similar stressful events in the future. Therefore, production and action for each of the stress mediators occurs within a limited time window and at specific anatomical locations. Nevertheless, such spatial and temporal niches of action of the different mediators are not strictly defined, but rather overlapping, which allows their actions to converge and generate a coordinated stress response (reviewed in (Joels and Baram, 2009)).

Dynamics and quality of molecular stress responses depend on type of stressor and duration of stress (Joels and Baram, 2009). For instance, physical stressors such as blood loss, trauma and cold rapidly recruit the brainstem and hypothalamic regions, whereas psychological stressors primarily engage 
stress mediators in brain regions dealing with emotions (the amygdala and the prefrontal cortex), learning and memory (the hippocampus) and decision-making (the prefrontal cortex).

The stress response can be characterized by two temporal waves of stress mediator actions (Figure 7). The first includes rapid actions of noradrenaline, serotonin, dopamine and CRH that stimulate vigilance, alertness, appraisal of the situation and the choice of an optimal strategy to face the challenge. GCs belong to the second temporal wave, which is most important for provoking long-term adaptive components of a stress response, such as memory formation. These classical effects of GCs in stress responses rely on alterations in gene transcription and are mediated via GR. In addition, GCs can modulate cell functions within few minutes, acting through non-genomic pathways including membrane-bound MR (Karst et al., 2005; Olijslagers et al., 2008).

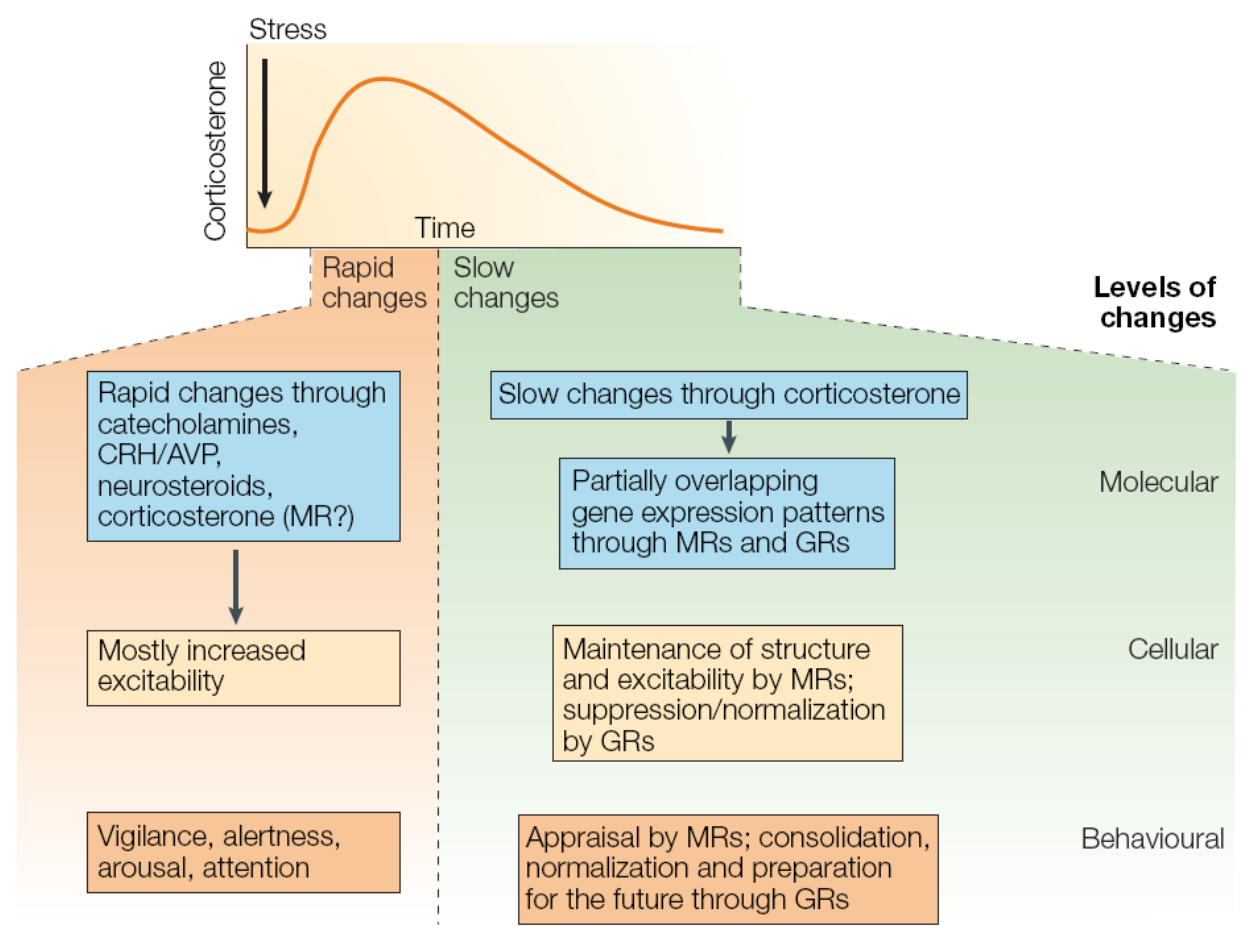

Figure 7. Time course of cellular responses to stress hormones. From (de Kloet et al., 2005).

Adaptive response to stress can lead to effective coping with a challenging situation and reinstate disturbed homeostasis. According to Sterling and Eyer, the process of adaptation to stress is defined as "allostasis", or "stability through change" (Sterling and Eyer, 1981). In contrast, if the stress response is inadequate or excessive and prolonged, the cost of reinstating homeostasis might become too high, which leads to a pathological condition termed "allostatic load". McEwen defined allostatic load as "wear and tear of the body and brain resulting from chronic hyperactivity or inactivity of the physiological systems that are normally involved in adaptation to environmental challenge" (McEwen 
and Gianaros, 2010). The concept of allostasis and allostatic load helps formally distinguish between adaptive, advantageous consequences of effective stress reaction and detrimental effects of chronic or inadequate stress due to the overload of the physiological functions.

Major targets of GC action include the central nervous system and most peripheral tissues, especially tissues involved in metabolism (liver, adipose tissue, muscle) and immune responses.

\section{GC effects in the brain}

Both corticosteroid receptors are involved in GC action in the brain during stress response. MR and GR are differentially distributed in brain. MRs are highly expressed in hippocampal neurons and the lateral septum and moderately expressed in the amygdala, PVN and locus coeruleus - regions that are involved in cognitive, emotional and neuroendocrine processing of stressful events. GRs are ubiquitously expressed in the brain, but enriched in the hippocampus, lateral septum and PVN.

MR is implicated in the appraisal process and the onset of the stress response. GR, which is only activated by large amounts of GCs, terminates the stress reactions, mobilizes the energy resources required for this purpose and facilitates recovery. Additionally GR promotes memory storage in preparation for future events.

One of the essential effects of GCs in the brain is to enhance learning and facilitate memory formation in preparation for similar stressful events in the future (Kim and Diamond, 2002). Therefore, the hippocampus, which is necessary for the formation of stable declarative memory in humans and spatial, or contextual, memory in rodents, is the main target of GCs in the brain, highly expressing both corticosterioid receptors. Hippocampal MR, which is activated with low amounts of GCs even during troughs of ultradian pulses, is necessary for maintaining basal neuronal activity, inducing longterm potentiation (LTP). By non-genomic action, GCs rapidly enhance excitability of CA1 pyramidal neurons via pre- and postsynaptic effects on the membrane MR (Olijslagers et al., 2008). On the other hand, activation of GR during stress or exposure to a novel environment causes a delayed suppression of neuronal excitability and synaptic plasticity by impairing LTP (Kim and Diamond, 2002). In the CA3 hippocampal area, a single stressor results in a delayed loss of apical dendrites but an enhanced basal dendritic tree (Kole et al., 2004).

Complex functional consequences of GC action in the hippocampus are highly dependent on the timing of events (de Kloet et al., 2005). Behavioral studies in rodents demonstrated that GR activation during stress is a prerequisite for the storage of relevant information. For instance, i.c.v. administration of GR antagonists immediately after the learning phase in a water maze impairs memory retrieval measured 24 hours later (Oitzl and de Kloet, 1992). GR ${ }^{\mathrm{dim} / \mathrm{dim}}$ mutant mice, which carry a point mutation that prevents GR dimerization and DNA binding, also show impairments in memory storage 
(Oitzl et al., 2001). Moreover, both MR and GR are important for control of neurogenesis in the dentate gyrus (Garcia et al., 2004). Brief exposure to stress temporarily suppresses proliferation and increases cell death, affecting the expression of several pro- and antiapoptotic genes in the dentate gyrus.

Importantly, an emotional, arousing situation promotes formation of long-lasting memories through recruitment of brain regions responsible for processing of emotional stimuli. Indeed, a number of studies showed that GC-mediated enhancement of memory storage involves noradrenergic activation in the basolateral amygdala (BLA) and bidirectional interactions between the BLA and the medial prefrontal cortex (mPFC) (Quirarte et al., 1997; Roozendaal et al., 2009).

To the contrary, exposure to chronic stress impairs subsequent attention and memory and can even induce profound amnesia (Bremner et al., 1996). Extensive evidence strongly suggests that GCinduced memory impairment is associated with drastic alterations in structure, organization and function of the hippocampus and related areas (reviewed in (Bremner et al., 1996; de Kloet et al., 2005; Kim and Diamond, 2002; McEwen and Sapolsky, 1995)). One of the most consistent effects of chronic stress is a reduction in the branching and length of CA3 pyramidal apical dendrites and a decreased number of synaptic contacts. Dendritic atrophy in the hippocampus can be prevented by NMDA receptor blockers, which implies a key role of glutamate in mediating these structural alterations (Magarinos et al., 1996). In addition, chronic exposure to high GC levels results in a longer-lasting reduction in proliferation and survival of progenitor cells in the dentate gyrus. This effect can be prevented by some antidepressants, e.g. tianeptine (Czeh et al., 2001). Such degenerative processes may explain the reduced hippocampal volume in patients with Cushing's disease and depression, pathologies characterized by chronic hypercortisolemia (Sheline et al., 1999).

Extensive animal studies and a wealth of strong correlative data in humans support the idea that disturbed HPA axis activity and chronic hypercortisolemia are risk factors for depression (de Kloet et al., 2005; Holsboer, 2000). A depression-like phenotype sensitive to antidepressant treatment was observed in mice lacking GR in limbic brain regions, which results in hyperactive HPA axis due to impaired negative feedback (Boyle et al., 2005). In accordance, the HPA axis of depressive patients poorly responds to suppressive action of Dex and stimulatory effects of CRH. Importantly, longitudinal studies showed that HPA axis activity is a good predictor for the relapse and remission of depressive symptoms (Zobel et al., 2001).

Stress and GC over-production affect the metabolic state of the body not only through GC effects in metabolic tissues, but also by modulating the activity of brain areas involved in appetite regulation and reward (Adam and Epel, 2007; Dallman et al., 2003; Koob, 2008; Parylak et al., 2011). The effect of stress on appetite depends on the type of stressor, its duration and intensity. Acute immobilization stress in rats leads to a transient decrease in food intake and body weight (Shimizu et al., 1989; Valles 
et al., 2000), whereas mild stressors, such as a gentle tail-pinch, rather promote spontaneous feeding (Morley et al., 1983). In line, Pijlman et al. showed that a physical stressor (foot shock) reduces consumption and preference for a saccharin drink compared to water, while emotional stress increased saccharin preference (Pijlman et al., 2003). In humans, chronic stress and negative emotional states can either enhance food intake and stimulate weight gain, as observed in most cases, or lead to weight loss through diminished food intake.

Food palatability plays a key role in the stress-related modulation of appetite (Adam and Epel, 2007). Using the Trier social stress test, Epel and others showed that those volunteers who responded with high cortisol were likely to consume more calories after the stressor, particularly of high fat food (Epel et al., 2001). In rats, food restriction combined with repetitive stress (but not food restriction alone) promoted greater palatable food intake over chow and this effect was sensitive to a non-specific opioid receptor antagonist (Boggiano et al., 2005). Studies on stress and addiction support the view that the dopaminergic reward system is involved in mediating the effect of stress on appetite. Indeed, uncontrollable stress or chronic GC administration increase intake of drugs of abuse, such as cocaine (Goeders, 2002). In contrast, mice with genetic inactivation of GR in the brain develop reduced motivation to self-administer cocaine compared to wild-type animals (Deroche-Gamonet et al., 2003). GR is expressed, and can be activated by stress exposure, in neurons of the dopaminergic reward circuit, including the substancia nigra and the ventral tegmental area (VTA) (Hensleigh and Pritchard, 2013). On the other hand, several lines of evidence suggest that GCs may influence the reward value of food via neuroendocrine mediators such as leptin, insulin and NPY (Adam and Epel, 2007).

\section{GC effects on metabolism}

Stress and GCs influence various peripheral metabolic pathways, mainly in the liver, adipose and muscle tissues (reviewed in (Vegiopoulos and Herzig, 2007)). During prolonged fasting or stress exposure, GCs coordinate increased energy supply to the brain by stimulating gluconeogenesis in hepatocytes, reducing glucose uptake by muscle and fat cells, and promoting lipolysis in the adipose tissue and protein break-down in muscles to release substrates for gluconeogenesis. Metabolic effects of chronic GC over-production are seen in patients suffering from Cushing's disease such as central obesity, increased breakdown of skeletal muscle mass, hyperglycemia, fatty liver development, elevated cholesterol, and insulin resistance (Shibli-Rahhal et al., 2006). In accordance, chronic social defeat leads to a pre-diabetes-like state in subordinate mice represented by hyperphagia and weight gain, increased leptin levels and dyslipidemia both on standard and high-fat diets (Sanghez et al., 2013). In humans, the Whitehall II study clearly established an association between lifetime stress exposure and the development of the metabolic syndrome and insulin resistance (Chandola et al., 2006). 
Liver-specific GR knockout mice display fasting hypoglycemia and are protected from streptozotocininduced hyperglycemia (Opherk et al., 2004). GCs promote hyperglycemia through GR-dependent induction of the gluconeogenic enzyme genes PEPCK and G6Pase (Vegiopoulos and Herzig, 2007). In addition, elevated GCs affect hepatic fat metabolism by promoting very-low-density lipoprotein (VLDL) production and secretion as well as triglyceride synthesis via fatty acid synthase (FAS) and acetyl-CoA carboxylase (ACC). Along with the inhibition of free fatty acid (FFA) beta-oxidation via interference with the activity of Acyl-CoA dehydrogenase, GCs trigger hepatic fat accumulation (steatosis) as well as systemically elevated blood lipid levels. GCs can also affect hepatic glucose metabolism via the brain. Kalsbeek and colleagues recently found that GC signaling specifically in neurons of the arcuate nuclei modulates hepatic insulin responsiveness via NPY and the sympathetic nervous system (Yi et al., 2012).

GCs play an essential role in regulation of fat deposition. Remarkably, treatment of obese Zucker rats with the GR antagonist RU486 or adrenalectomy reverses the obese phenotype in these animals (Livingstone et al., 2000). GCs differentially affect distinct fat depots. In peripheral fat depots, GCs increase lipolysis by inducing hormone-sensitive lipase (HSL) and inhibiting lipoprotein lipase (LPL) activity. In contrast, GCs promote pre-adipocyte differentiation, pro-lipogenic pathway activity, and thereby cellular hypertrophy in central (abdominal) fat. Interestingly, MR is critical for adipogenic effects of GCs (Marzolla et al., 2012). Moreover, GCs were shown to directly influence insulin sensitivity in adipocytes by interfering with components of the insulin-signaling cascade.

In the muscle, GCs inhibit glucose uptake and glycogen synthesis. On the other hand, they suppress protein synthesis while promoting protein degradation and amino acid export. These catabolic effects are exerted through modulation of several molecular pathways, including suppression of the insulin/IGF-1 signaling, which leads to decreased glucose uptake and reduced glycogen and protein synthesis; activation of the ubiquitin-proteasome pathway; and secretion of myostatin, an inhibitor of muscle growth (Vegiopoulos and Herzig, 2007).

\section{Regulation of the negative GC feedback}

During ultradian pulses or acute stress responses, GCs effectively suppress their own production through inhibitory action at the levels of the PVN and anterior pituitary (Herman et al., 2012). Fast non-genomic effects of GCs on CRH-producing PVN neurons require endocannabinoid release that inhibits presynaptic glutamatergic signaling (Evanson et al., 2010), likely mediated via a membranebound GR (Laryea et al., 2013). In addition, limbic structures such as the hippocampus and mPFC are involved in inhibition of the HPA axis and termination of stress responses (McEwen and Sapolsky, 1995). Indeed, genetic deletion of the GR in the limbic forebrain, but not in the PVN, causes delayed shut-off of HPA axis responses to psychogenic stressors and impaired Dex suppression (Boyle et al., 2005). 
Chronic stress causes a persistent increase of $P O M C$ mRNA in anterior pituitary, adrenal hyperplasia and GC over-production. Interestingly, at the level of the PVN, the central activation of HPA activity is taken over by a predominant AVP rather than CRH drive, as shown in an arthritis model of chronic stress (Chowdrey et al., 1995) and in repeated restraint stress (Ma et al., 1997). Chronically elevated GC levels lead to altered sensitivity of the HPA axis to negative feedback. Chronic psychosocial stress (daily social defeat) promotes down-regulation of GR in the hippocampus and pituitary and resistance to suppressive effect of Dex on GC production (Bartolomucci et al., 2004). Interestingly, chronically stressed rats exhibit facilitated ACTH responses to acute stress ((Dallman et al., 2003) and refs within). Moreover, Dallman and co-workers suggest that, under conditions of chronic stress, an indirect GC feedback takes place as well, being mediated by $\mathrm{GC}$-induced metabolic alterations (Dallman et al., 2004).

\section{Developmental programming of HPA axis sensitivity}

Early life experiences can program HPA axis reactivity and vulnerability to stressful events later in life. Pioneering studies by S. Levine demonstrated that already brief separations of rat pups from the mother reduces emotional and neuroendocrine reactivity to common stressors in adulthood (Levine, 1957), which is likely caused by increased maternal care that the pups received upon their return. Indeed, the offspring of high licking and grooming mothers showed attenuated activity of the stress axis, improved cognitive performance in a spatial-learning test and reduced anxiety-like behavior in adulthood (Liu et al., 1997). The programming effect of maternal behavior is associated with reduced DNA methylation in the promoter of the $N r 3 c l$ (GR) gene and hence higher hippocampal GR expression in pups (Weaver et al., 2004). On the other hand, repeated daily mother-offspring separations (i.e. maternal deprivation/neglect) produced a phenotype later in life that was characterized by enhanced emotional and HPA axis responsiveness to brief stressors (Ladd et al., 2004).

\subsubsection{The circadian clock and stress response}

\section{A role of the circadian clock in acute stress response}

Responsiveness of the HPA axis to stress varies around the clock. Restraint stress as well stress by ether or novel environment has been reported to produce a greater corticosterone response at the beginning of the active phase in rodents (Dunn et al., 1972; Kant et al., 1986; Sage et al., 2001). Lesioning the SCN causes a loss in the diurnal difference in this response, but not the ability to respond itself (Sage et al., 2001). In accordance, mice lacking VIP in the hypothalamus and adrenals loose circadian rhythms of ACTH and CORT, but are capable of mounting a normal GC response to acute stress (Loh et al., 2008). 
Acute immobilization stress induces a more intense grooming behavior in Perl mutant mice, which is accompanied by elevated expression of $\mathrm{Crh}$ in the PVN (Zhang et al., 2011). However, CORT responses to stress and GR expression in PVN were not affected by Perl deficiency implying a contribution of GC-independent mechanisms. Similarly, Bmall-deficient mice as well as mice with targeted down-regulation of Bmall transcript in adrenal glands are able to up-regulate GC production in response to acute restraint stress (Curtis et al., 2007; Son et al., 2008).

Is exposure to acute stress capable of resetting the circadian clock in vivo? Yamamoto et al. demonstrated that both behavioral circadian rhythms in constant darkness as well as the central clocks in peripheral tissues are largely resilient to the effect of 1-h restraint stress exposure (Yamamoto et al., 2005). Among eleven clock genes studied, only Perl was up-regulated in the liver and other organs after acute stress or CORT injection. Further studies are needed to understand a physiological significance of stress-induced Perl responses independently of its role in the circadian clock.

\subsubsection{When good things turn bad: interplay between the circadian clock and GCs in chronic stress and pathology}

Animal studies and clinical data suggest that chronic stress can impair circadian and ultradian oscillations along the HPA axis and GC-mediated synchronization of tissue clocks. It is important to note that chronic stress effects are complex and, in addition to GC hormones, rely on multiple stress mediators (Joels and Baram, 2009).

Not only overall daily GC secretion is elevated in chronic stress, but the frequency of ultradian GC pulses significantly increases. Lightman and colleagues revealed that rats with adjuvant-induced arthritis, which represents a model of chronic hyperactivation of the HPA axis, show an almost twofold increase in the number of GC pulses per day (Windle et al., 2001). Interestingly, when the frequency of pulsatility increases, there is an increased proportion of time when the animals are in a stress nonresponsive state, giving rise to apparent stress hyporesponsiveness (Windle et al., 2001). Similarly, neonatal stress can exert long-term programming effects on the mechanisms generating basal pulsatile HPA activity in adult animals. Endotoxin injection in neonatal rats results in both an increased frequency and amplitude of GC pulses (Shanks et al., 2000).

It was shown that chronic stress impairs functioning of circadian clocks in the brain, including the SCN. Chronic restraint stress alters circadian behavioral rhythms through a change in circadian gene expression of Per 2 and GSK-3 $\beta$ phosphorylation in the SCN (Kinoshita et al., 2012). Jing et al. showed that chronic unpredictable stress in mice leads to depression-like behavior and a dampened amplitude of PER2 expression in the SCN, which was restored after termination of stress (Jiang et al., 2011). This is in line with blunted locomotor rhythms in mice exposed to chronic unpredictable stress (Solberg et al., 1999). Furthermore, CLOCK, but not BMAL1, protein expression in the hippocampus 
was altered in the stressed mice (Jiang et al., 2013). However, GC levels were not measured in the mentioned studies; therefore a contribution of GC-GR signaling to PER2 and CLOCK rhythm deregulation remains unclear and potential effects of other stress mediators cannot be excluded. It still remains unclear how circadian clock gene deficiencies would affect an animal's sensitivity to chronic stress and activity of HPA axis.

Chronic stress responses associated with exposure to abnormal light-dark schedule do not necessarily depend on deregulated rhythms in the SCN. Interestingly, LeGates et al. recently found that another type of a chronic stressor - aberrant light exposure (ultradian 7-hour light-dark cycles) - does not affect central clock rhythms, but still induces GC over-production and depression-like behavior in mice, presumably via modulation of neural activity in stress response-related brain areas, such as the amygdala (LeGates et al., 2012).

Finally, it was recently shown that chronic mild stress can affect rhythms of hepatic core clock genes and key metabolic genes (Takahashi et al., 2013), which suggests that chronic stress, likely via elevated GC levels, may also alter peripheral clock stability. Indeed, disturbance of GC rhythms in chronically stressed people may result in the loss of intercellular synchrony in tissue clocks, as predicted by mathematical modeling (Mavroudis et al., 2012).

A number of pathological conditions are associated with impaired GC production and alterations in circadian rhythms. Moreover, chronic stress, which per se affects the circadian clock and GC rhythms, can promote as well as be a consequence of many pathological conditions.

The loss of a normal diurnal rhythm is the hallmark of Cushing's syndrome of different etiology (Pirich and Vierhapper, 1988; Yaneva et al., 2004). Few studies and case reports have also revealed patients with shifted or inverted cortisol rhythms (Ambrosi et al., 1985; Olsen et al., 1978; Shimatsu et al., 1988; Xu et al., 1991). Interestingly GC-mediated signaling in tissues of Cushing's patients might still be rhythmic, since the circadian rhythm of GR does not significantly deviate from that of normal subjects (Xu et al., 1991).

Diverse circadian abnormalities have been observed in depressive subjects and animal models of depression including deregulated diurnal GC rhythms (reviewed in (Albrecht, 2013a; Turek, 2007). Patients with major depressive disorder display disruptions of daily rhythms of clock gene expression in blood cells as well as flattened altered plasma cortisol rhythms (Jarcho et al., 2013; Li et al., 2013). Similarly, lower morning cortisol peaks and flattened diurnal rhythms of plasma cortisol were reported in Holocaust survivors with posttraumatic stress disorder, which is, in contrast to major depression, characterized by decreased cortisol production and hypersensitivity of the HPA axis (Yehuda et al., 2005). A postmortem study found less AVP mRNA and higher AVP immunoreactivity in the SCN in patients with major depression (Zhou et al., 2001), which suggests that not only peripheral clocks, but 
also central clock function is likely to be affected. In accordance, SCN lesioning in rats that leads to arrhythmic GC production, reduces time spent immobile in the forced swim test (Tataroglu et al., 2004), indicating an increased resilience to stress (Castagne et al., 2011).

GC rhythms are deregulated in many cancer patients (reviewed in (Mormont and Levi, 1997)) and have been correlated with survival, as shown for breast cancer (Sephton et al., 2000). However little is known about causes of impaired GC rhythms in cancer and about the mechanisms that link GCs and tumorigenesis. Both direct action of GCs on tumor cells or indirect influence via regulation of the immune system and metabolism are possible (reviewed in (Volden and Conzen, 2013). Intimate interactions have been shown between GC rhythms and cell cycle progression (reviewed in (Dickmeis and Foulkes, 2011) highlighting a potential importance of deregulated GC rhythmicity in initiation and progression of tumor growth.

Chronic inflammation, a hallmark of autoimmune disorders, is often associated with elevated GC production and flattening of diurnal GC rhythms, as shown in several animal models such experimental autoimmune encephalomyelitis (a model of multiple sclerosis) (Buenafe, 2012), lupusprone MRL/MP-fas ${ }^{l p r}$ mice (Lechner et al., 2000) and rats with adjuvant-induced arthritis (Sarlis et al., 1992). Interestingly, Lechner et al. found that increased GC production during the light phase in MRL/MP- $f a s^{l p r}$ mice was not associated with a corresponding surge of plasma ACTH levels (Lechner et al., 2000), which may point to altered ACTH sensitivity. On the other hand, diurnal HPA axis activity remains normal in patients with rheumatoid arthritis, although circadian variation in severity of symptoms and rhythmic secretion of pro-inflammatory cytokines is preserved and in anti-phase with plasma cortisol levels (Crofford et al., 1997; Harkness et al., 1982; Kirwan et al., 2010).

Aberrant GC rhythms were found in metabolic diseases (Nader et al., 2010). Obesity is associated with a flattening of the diurnal rhythm; plasma cortisol levels are slightly increased during the trough, although they are normal or low in the morning ((Buren et al., 2007) and refs within). Moreover, obese Zucker rats lack a circadian rhythm of 11beta-HSD1 gene expression in the hippocampus, which may contribute to increased HPA axis activity (Buren et al., 2007). Similarly, obese mice kept on high-fat diet as well as overweight and obese children show substantial down-regulation of overall GC production (Kjolhede et al., 2013; Kohsaka et al., 2007). Interestingly, plasma cortisol levels seem to be not affected in patients with cirrhosis, but the timing of their diurnal cortisol peak is considerably (2-3 h) delayed (Montagnese et al., 2011). Finally, metabolic abnormalities such as defective glucose utilization and obesity coincide with impaired HPA axis activity and altered GC rhythms in Per1 and Per2 mutant mice (Dallmann et al., 2006; Yang et al., 2009).

In conclusion, despite the fact that disturbances of the circadian clock and daily GC rhythms are welldocumented in various diseases, it is still a long way to go until causative interactions are revealed to establish potential molecular targets for chronotherapy. 
1.6. Publication: "Physiology of the adrenal and liver circadian clocks"

Alexei Leliavski and Henrik Oster

In: Circadian Medicine (Wiley-Blackwell, Ed.: C.S. Colwell), in press. 


\title{
Physiology of the adrenal and liver circadian clocks
}

\author{
Alexei Leliavski $^{1} \&$ Henrik Oster ${ }^{1,2, *}$ \\ ${ }^{1}$ Circadian Rhythms Group, Max Planck Institute for Biophysical Chemistry, Göttingen, Germany \\ ${ }^{2}$ Medical Department I, University of Lübeck, Germany \\ *Correspondence to henrik.oster@uksh.de / tel. ++49-451-500-2306
}

\begin{abstract}
In mammals cellular clocks are found in most tissues of the body. While behavioral rhythms are determined by the master clock of the suprachiasmatic nuclei (SCN), peripheral clocks regulate local physiology via the rhythmic orchestration of transcriptional networks. Adrenal and liver oscillators are major regulators of endocrine and metabolic rhythms. In the adrenal diurnal secretion of gluco- and mineralocorticoids is controlled by both local and central means. In the liver endogenous and systemic rhythms are integrated and together regulate various aspects of liver physiology including energy metabolism and the regulation of biotransformation. The assessment of peripheral clock regulation has important clinical implications including the optimization of drug efficacy and the avoidance of unwanted side effects.
\end{abstract}

\section{Introduction}

In most organisms, various aspects of physiology and behavior including metabolic, endocrine and immune functions show circadian rhythms that are governed by endogenous molecular clocks. In mammals, it is assumed that (almost) every cell of the body contains its own circadian oscillator (Dibner et al., 2010) based on interlocked transcriptional-translational feedback loops that are tightly coupled with the metabolic state of the cell (Zhang and Kay, 2010). Depending on tissue, the molecular clock orchestrates circadian oscillations of $5-10 \%$ of the cellular transcriptome, among them genes that encode key rate-limiting enzymes and regulatory proteins of cellular physiology (Ko and Takahashi, 2006).

Circadian clocks are able to sustain rhythmicity with a period close to 24 hours even in constant conditions devoid of any external timing signals. Because this endogenous period differs slightly from 24 hours, under natural conditions synchronizing stimuli (Zeitgeber) such as light and food play an essential role in adjusting the period of endogenous clocks to geophysical time. To organize such entrainment at the organismal level, a hierarchy between different tissue clocks has developed. The hypothalamic suprachiasmatic nuclei (SCN) are 
considered as a central circadian pacemaker which receives light input directly from a specific subpopulation of photosensitive ganglion cells in the retina and transmits this information downstream to synchronize single cell oscillators in various tissues (Dibner et al., 2010). The SCN clock is necessary to maintain synchrony amongst tissue clocks under Zeitgeber-free conditions (Stephan and Zucker, 1972), but peripheral clocks can maintain rhythmicity in explants (Yoo et al., 2004) or under entrained conditions (Oster et al., 2006b). Light can modulate circadian clocks in certain peripheral tissues, including the adrenal gland and the liver. Much stronger peripheral synchronizers, however, are food intake, temperature changes or adrenal glucocorticoids (Dibner et al., 2010).

The recent development of genetic tools allowing the tissue-specific deletion of clock genes has provided new insight into the contribution of peripheral clocks to the regulation of physiological rhythms. In this chapter, we describe circadian physiology of two organs heavily involved in metabolic regulation and energy homeostasis - the liver and the adrenal gland. Both tissues harbor robust circadian clocks that possess partial autonomy from the SCN. We discuss the functions of these oscillators, potential communication routes between them and the $\mathrm{SCN}$, and how their disturbance may promote the development of diseases.

\section{Circadian control of adrenal function}

The adrenal is one of the eight major endocrine glands in mammals. Through its hormones-glucocorticoids mineralocorticoids

(MCs)

(GCs), and catecholamines (CAs) - it is heavily involved in the regulation of physiological processes such as stress responses, energy metabolism, blood pressure, learning and memory, and the immune system (Fig. 1). During development adrenocortical precursor cells originate from the coelomic epithelium as part of the adrenogonadal primordia whilst the adrenal medulla is formed by neural crest cells, which subsequently invade the expanding population of cortical cells. The adrenal cortex of adult mammals has two clearly distinguished subregions - the zona glomerulosa which produces mineralocorticoid aldosterone and the zona fasciculata responsible for glucocorticoid synthesis (mainly cortisol in humans and corticosterone in rodents). In primates, including humans, the adrenal cortex also contributes to the production of sex steroid precursors (dehydroepiandrosterone (DHEA) and DHEA-sulfate) synthesized in the third, most inner, cortical sub-region, the zona reticularis (Havelock et al., 2004). In contrast, the murine adrenal cortex lacks a clearly distinguished zona reticularis and cannot produce androgens due to the absence of $17 \alpha$-hydroxylase. The medulla forms the core of the adrenal gland, where acetylcholine-responsive chromaffin cells synthesize the stress hormones epinephrine ( $70 \%$ of cells) and norepinephrine $(25 \%$ of cells). In humans chromaffin cell synthesize both CAs, while in rodents the hormones are produced by two separate sub-populations of cells. A third sub-population of medullar cells, small granule-containing cells (approx. $4 \%$ in mice), produce further peptides such as met-enkephalin, substance $P$, 
neuropeptide $\mathrm{Y}$, neurotensin and chromogranin A.

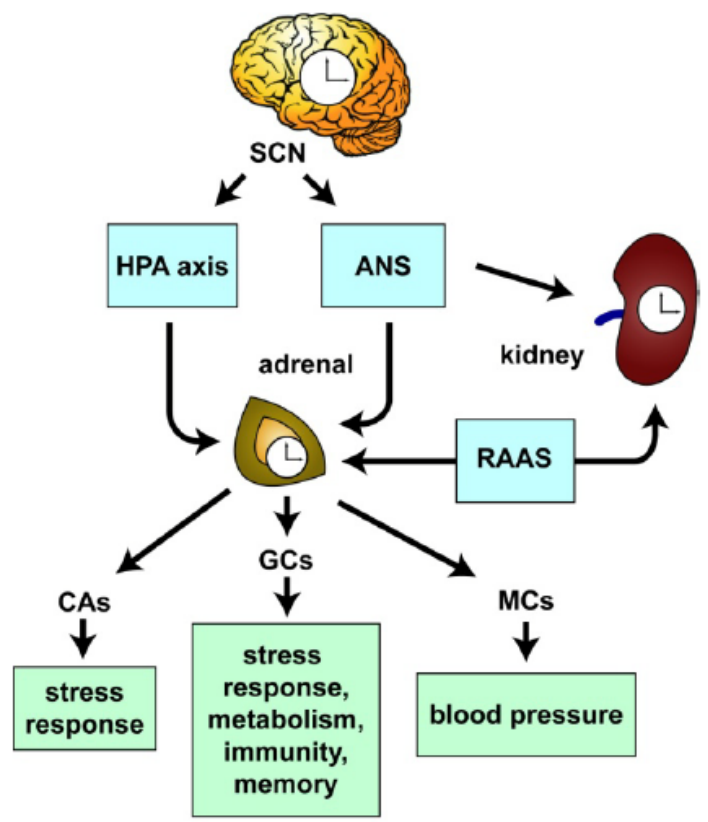

Figure 1. Circadian control of hormonal production by the adrenal gland. For details see text.

\section{Glucocorticoids (GCs)}

Under basal conditions adrenocortical GC secretion follows robust circadian and ultradian (1-2 pulses/hour) oscillations. The circadian peak of GC production is observed at the beginning of the activity phase, i.e. during early morning in humans and other diurnal animals and the early night in nocturnal species such as most rodents. The $\mathrm{SCN}$ neurons seem to be indispensable for the generation of the circadian GC rhythm component (Stephan and Zucker, 1972), while ultradian GC pulses are preserved in $\mathrm{SCN}$ lesioned animals (Waite et al., 2012). Timing signals from the SCN reach the adrenal via two confirmed modes, the hypothalamic-pituitary-adrenal (HPA) axis and the autonomic nervous system (ANS) (Fig. 1). The first node of the HPA axis is the hypothalamic paraventricular nucleus (PVN), which receives stress-driven signals from the brainstem structures and forebrain limbic circuits (Ulrich-Lai and Herman, 2009). The SCN sends indirect projections to PVN neurons, mainly via inhibitory projections to the sub-paraventricular zone and the dorsomedial hypothalamus (Buijs et al., 1993). PVN neurons produce corticotropin-releasing hormone and vasopressin, which stimulate corticotropic cells in the pituitary to secrete adrenocorticotropic hormone (ACTH) from secretory granules. In adrenocortical cells ACTH up-regulates streroidogenesis via steroidogenic acute regulatory protein (StAR)-mediated increase of cholesterol delivery to the inner mitochondrial membrane for conversion by cholesterol side-chain cleavage enzyme (CYP11A1). Long-term effects of ACTH include an increase in expression of other steroidogenic enzymes (CYP17, CYP21A1, CYP11B1) and improved cholesterol uptake via low and high density lipoprotein receptors. The system is kept under control via negative feedback: GCs suppress their own production acting through the glucocorticoid receptor at the level of the pituitary and the PVN. On the other hand, SCN neurons regulate rhythmic functioning of the adrenal gland through the autonomic nervous system (ANS) and hypophysectomy does ablate GC rhythms only in combination with adrenal denervation. The multisynaptic ANS pathway consists of pre-autonomic PVN neurons, sympathetic preganglionic intermediolateral neurons of the spinal cord, 
and splanchnic innervation of the adrenal gland (Buijs et al., 1993). ANS signals can affect adrenal physiology via modulation of adrenal clock gene activity. A 1-hour light pulse can activate $\mathrm{Per}$ gene expression in the adrenal and drive slow ACTH-independent $\mathrm{GC}$ release, and this response requires intact adrenal innervation (Ishida et al., 2005). SCN-adrenal neural connections are essential for maintaining circadian regulation of adrenal sensitivity to ACTH in vivo (Dallman et al., 1978).

\section{Mineralocorticoids (MCs)}

The mineralocorticoid aldosterone, a key regulator of blood pressure, exhibits daily oscillations with a peak in the active phase. Aldosterone production is under control of the renin-angiotensin-aldosterone system (RAAS) (Bader, 2010). In brief, the peptidase renin released by renal juxtaglomerular cells in response to low blood volume converts its substrate, angiotensinogen, to angiotensin I (Ang I), which in turn is cleaved by the angiotensinconverting enzyme to liberate Ang II. Ang II stimulates aldosterone synthesis in the adrenal cortex. Aldosterone binding to the mineralocorticoid receptor in renal epithelial cells reduces extracellular fluid volume via stimulation of sodium reabsorption and potassium excretion by the kidneys, thus lowering blood pressure. Plasma renin activity, ACE and Ang II levels display circadian variation both in rodents and humans, suggesting that circadian control of the RAAS by the SCN or renal clocks may contribute to aldosterone rhythmicity (Guo et al., 2005).

\section{Catecholamines (CAs)}

Both epinephrine and norepinephrine display diurnal variations in blood and urine samples from unstressed humans and mice, with a peak in the beginning of the active phase (Linsell et al., 1985). Daily rhythms of CA release from the adrenal medulla parallel diurnal changes in sympathetic tone, suggesting central regulation of sympathetic adrenomedullary system activity by the SCN. In line with this, SCN lesions in rats abolish circadian rhythms of heart rate and blood pressure, mainly controlled by the sympathetic system, without affecting activity and sleep-wake cycles (Janssen et al., 1994).

\section{Adrenal clocks}

The above findings demonstrate that the SCN clock is critical for maintaining daily rhythmicity of adrenal hormones. However, several lines of evidence suggest that the adrenal gland itself harbors an intrinsic circadian oscillator. Firstly, clock gene expression in the adrenal of rodents and primates is highly rhythmic (Oster et al., 2006a; Oster et al., 2006b). Interestingly, circadian rhythms in the expression of clock genes are different in different adrenal regions (Oster et al., 2006b) (Fig. 2). Moreover, approximately $5 \%$ of the adrenal transcriptome is expressed in a circadian fashion (Oster et al., 2006a), including genes involved in cholesterol uptake and transport, regulators of steroid biosynthesis, ACTH signaling, and CA metabolism (Maoa, Nr4a2, Pnmt). Circadian variations in expression of key transcripts involved in CA synthesis (Th, Pnmt) and reuptake (Slc6a2) were also found in an independent 
microarray study of monkey adrenals (Lemos et al., 2006). Interestingly, it has been shown that StAR, a key rate-limiting cholesterol transporter, is also rhythmically expressed and under direct regulation by the clock machinery (Son et al., 2008). In 1964 Andrews and Falk demonstrated that cultured hamster adrenal glands maintain circadian patterns of respiration and steroid secretion ex vivo (Andrews and Folk, 1964). Similarly, adrenocortical Y-1 cells display a circadian rhythm of GC synthesis in vitro (Son et al., 2008) and transplanted wild-type adrenal grafts can maintain rhythmic clock gene expression and glucocorticoid production under entrained conditions in clock-deficient transgenic mice (Oster et al., 2006b).

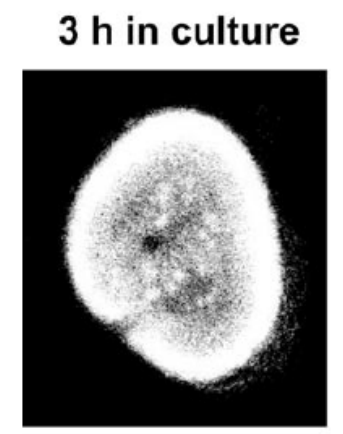

\section{$17 \mathrm{~h}$ in culture}

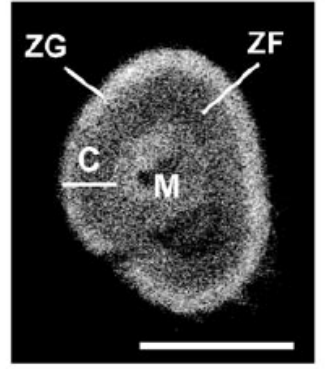

Figure 2. The adrenal clock is sustained in culture. Luminescence imaging of PER $2:: L U C$ mouse adrenal slices. $\mathrm{C}$-cortex, $\mathrm{M}-$ medulla, $\mathrm{ZG}$-zona glomerulosa, ZF-zona fasciculata; scale bar is $1 \mathrm{~mm}$.

\section{Local control of MC rhythms}

Adrenalectomy severely affects circadian variations in blood pressure and heart rate in wild-type mice. Moreover, the components of the RAAS are also produced locally in the adrenal cortex in response to $\mathrm{ACTH}$, regulating sensitivity of adrenocortical cells to Ang II. However it is unclear whether the sensitivity to Ang II changes throughout the day. Recently Okamura and colleagues provided strong evidence for a role of the adrenal circadian clock in aldosteronemediated regulation of blood pressure (Doi et al., 2010). Cryl/2 double deficient mice lacking a functional circadian clock display abnormally high levels of plasma aldosterone. This effect is due to overexpression of Hsd3b6 in Cry mutant adrenals, which encodes a steroidogenic enzyme that acts upstream of aldosterone synthesis. Hsd $3 b 6$ is expressed exclusively in aldosterone-producing zona glomerulosa cells. When fed a high salt diet Cry-null mice become hypertensive. This elevation in blood pressure is aldosterone-dependent and can be reversed by treatment with the aldosterone blocker eplerenone. It remains to be shown whether the adrenocortical clock itself is sufficient to maintain aldosterone rhythmicity or whether it coordinates the sensitivity of the steroidogenic machinery to systemically driven rhythmic cues.

\section{Local control of GC rhythms}

In vitro data suggest that the adrenal clock is essential for gating the adrenal responsiveness to $\mathrm{ACTH}$ stimulation. In cultured clock-deficient (Per2/Cryl double mutant) adrenal slice cultures circadian variations in the corticoid response to $\mathrm{ACTH}$ stimulation is abolished (Oster et al., 2006b) and the magnitude of this response is also highly compromised upon suppression of Bmall (Son et al., 2008). Two different approaches have been used so far to dissect a functional role of the adrenal clock in vivo. Our group performed adrenal 
transplantations between arrhythmic Per2/Cryl double mutant and wild-type mice (Oster et al., 2006b). We found that an intact adrenal clock is sufficient for maintaining GC rhythmicity in a clockdeficient host under 12h:12h light/dark conditions, but rhythms are lost after removing the external Zeitgeber. Alternatively, Son and co-workers performed genetic ablation of the adrenal clock (Son et al., 2008), knocking down Bmall by overexpressing an antisense transcript under the control of the $\mathrm{ACTH}$ receptor gene $(\mathrm{Mc} 2 r)$ promoter. In these mice GC rhythms were strongly dampened in constant darkness, but not in light-dark conditions, suggesting that the adrenal clock is required for maintaining high amplitude GC oscillations.

Interestingly under certain conditions the adrenal clock is also involved in the synchronization of clocks in other peripheral tissues. Fast traveling over several time zones results in misalignment of endogenous clocks. This is reflected in a range of symptoms affecting sleep timing, appetite and digestion, cognitive performance, and immune responses, comprised under the term jetlag. During jetlag circadian clocks of different organs (including the liver) reentrain at different speed, creating a transient state of internal desynchrony. Adrenal clocks coordinate global resetting via rhythmic regulation of GC levels. Pharmacologically shifting GC profiles prior to a jetlag can promote the re-entrainment process, at least in mice (Kiessling et al., 2010).

Of note GC production has two general modes of control. Besides circadian modulation of basal GC levels there is acute induction of GC synthesis by stressful stimuli-physical, psychological, inflammatory, or metabolic. Both modes can potentially influence each other. Circadian changes may define an activation threshold of the HPA axis to acute stressors and, vice versa, stress-induced GC surges may serve as resetting signals for other peripheral clocks. It is known that the intensity of stress-induced GC responses depends on the time of day, but the results to date are far from being conclusive. Some reports in rodents and humans show that HPA axis responses are more pronounced at the beginning of the activity phase (Buijs et al., 1993), while other reports found an opposite pattern (Kalsbeek et al., 2003) or failed to detect any diurnal changes. SCN lesion studies provide further evidence for a role of the central clock in maintaining daily stress sensitivity (Buijs et al., 1993). Interestingly, the type of stress stimulus appears critical in this context. While novelty exposure in rats elicits maximal ACTH and corticosterone responses in the early morning, insulininduced hypoglycemia stimulated GC release peaks in the early night (Kalsbeek et al., 2003). These findings suggest the existence of at least two different clockcontrolled stress pathways that can activate adrenal GC secretion.

\section{Circadian control of liver function}

The liver is-after the skin - the largest organ of the body. Its primary function is the metabolization of nutrients and the storage of glucose-and, to a lesser extent, lipidsas energy fuels for the body. It further shares an important role with the kidneys in detoxification and excretion processes. More 
recently, the liver has also been identified as an endocrine organ, secreting hormones such as insulin-like growth factor-1 (IGF-1), angiotensinogen, thrombopoeitin, and hepcidin. Structurally the mammalian liver consists of several large lobes (four in humans and rodents). Roughly $60 \%$ of the cells in the liver are made up of hepatocytes of parenchymal origin. Hepatocytes are also the largest cells of the liver, making up to $80 \%$ of the liver volume. Other cells include endothelial, macrophage-like Kupffer, and hepatic stellate cells (also known as Ito cells). Like many other growth factors IGF-1 secretion follows a circadian rhythm with peak levels during the rest phase. Circadian rhythms of thrombopoietin, but not of hepcidin and angiotensinogen, have been described (Levi and Schibler, 2007). Diurnal variations of blood pressure seem to rather depend on rhythmic release of vasopressin from the hypothalamus and aldosterone from the adrenal (see above).

The functions of the liver in energy metabolism and detoxification are tightly linked to the diurnal variation of food intake. In fact, transcriptome studies in rodents suggest that food intake may be a stronger synchronizer of liver transcriptome rhythms than the circadian clock itself (Vollmers et al., 2009). Together with nutrients numerous substances are taken up, that either need to be chemically modified to be useful to the body, or that-because of their toxicityhave to be removed again from the system. The liver prepares for these needs by regulating its transcriptional machinery to ready the enzymatic armory in times of need while saving energy during fasting times, e.g. during the daily rest phase. Of note, feeding time is also a strong synchronizer of peripheral clocks and the liver clock can phase-reset in response to regularly timed food intake, an effect that becomes important during jetlag when adjusting mealtime can have dramatic effects on the re-entrainment process at the destination of travel (Angeles-Castellanos et al., 2011).

\section{Glucose metabolism}

Glucose metabolism is probably the best described rhythmic process in the liver, but its regulation is highly complex, influenced by both internal and external timing cues (Fig. 3). In the postprandial phase higher order carbohydrates are broken down to fructose and glucose and transported to the liver via the blood. Upon entry into hepatocytes glucose is quickly converted into glucose-6-phosphate-and by this taken out of the equilibrium - and either broken down to pyruvate for further conversion or stored as glycogen. Glycogen biosynthesis is high during the active (feeding) phase and glycogen breakdown becomes a major source of energy during the inactive (fasting) period of the day, i.e. the night in humans or the day in nocturnal rodents (Kohsaka and Bass, 2007). The uptake into and release of glucose from liver storage is regulated by the insulin/glucagon endocrine system that responds to changes in blood glucose levels. Other hormones such as cortisol and leptin/ghrelin, most of which are under the control of the circadian clock, also play a role (Levi et al., 2007). Cortisol induces gluconeogenesis, the de novo synthesis of glucose from pyruvate, and promotes the release of glucose into the blood. Leptin and ghrelin impinge on glucose metabolism 
primarily via indirect means, regulating appetite centers in the basal hypothalamus (Leproult and Van Cauter, 2010).

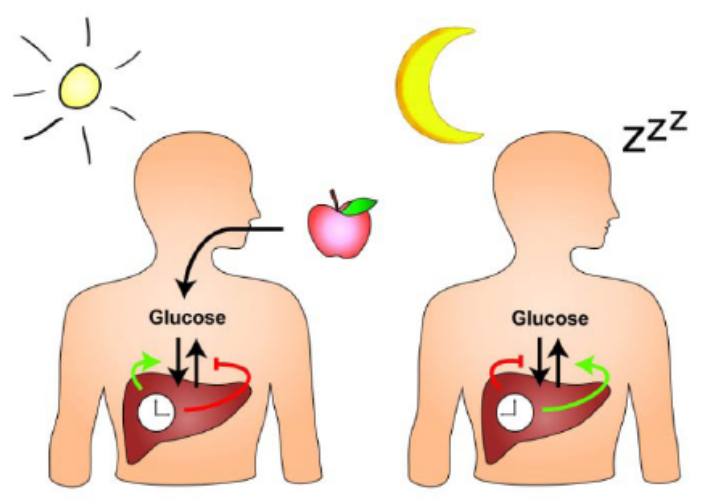

Figure 3. Liver circadian clocks regulate blood glucose levels by timing of glucose uptake and release.

\section{Lipid metabolism}

Though circadian aspects of lipid metabolism have mostly been described for adipose tissues, similar to glucose, lipid metabolism in liver is tightly coupled to food intake. Lipids are transported in the blood in the form of lipoproteins. Upon food intake chylomicrons are assembled in the intestinal mucosa. Most chylomicrons are taken up from muscle and adipose tissues, but the so called remnants reach the liver where they can be used for conversion into lipid derivatives or for break-down into acetylCoA. Acetyl-CoA is also the substrate for endogenous cholesterol. Cholesterol biosynthesis is robustly rhythmic with a peak during the late active phase (i.e. the evening in humans and the early morning in rats). Remnants become problematic when lipids are taken up excessively or at the wrong time of day, e.g. under high fat (Western) diet conditions or in patients with night eating syndrome. Circadian disruption, which is also seen in night shift workers or during jetlag, has strong effects on liver lipid metabolism, promoting excessive lipogenesis and the development of nonalcoholic fatty liver disease (NAFLD) (Levi and Schibler, 2007).

\section{Detoxification}

The rhythmic regulation of biotransformation and detoxification processes in the liver has important implications for the clinics as a critical determinant of drug pharmacokinetics, dynamics and the manifestation of side effects (Fig. 4). As just one example, the lethal toxicity of a fixed dose of the anesthetic halothane in mice can vary between $5 \%$ and $76 \%$, depending on the time of day when the drug is given (Levi and Schibler, 2007). Similar effects are described for various anti-cancer agents and antibiotics. Circadian variations in the efficacy of drugs have been documented for all relevant parameters, absorption, distribution, metabolism, and elimination $(A D M E)$, most of which are regulated by the liver (or, sometimes, the kidneys). This has giving rise to the field of chronopharmacology (Levi and Schibler, 2007). Of note, circadian rhythms differ between males and females and amongst individuals (of different chronotypes). Therefore easy and efficient means of determining endogenous clock phase in patients are needed to better implement circadian knowledge into the clinical practice. 


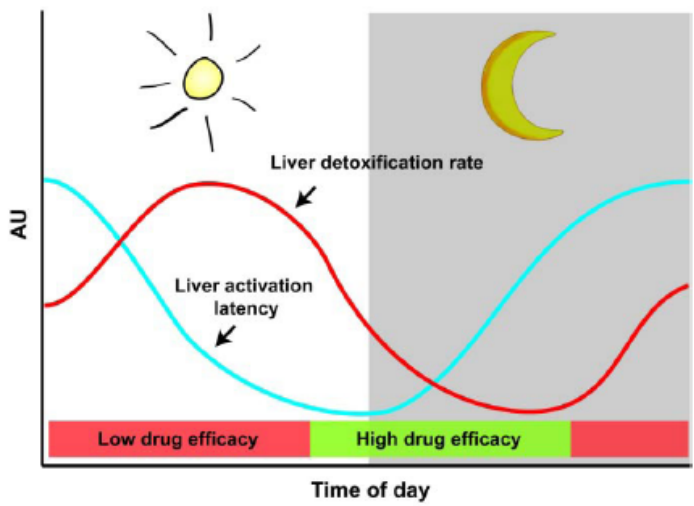

Figure 4. The efficacy of many drugs dependents is determined by daily profiles of detoxification rate and activation latency in liver.

\section{Hepatocyte clocks}

Hepatocyte clocks have been excessively studied in rodents, while very little is known about clocks in other cells of the liver. The

hepatocyte clockwork comprises the same set of clock genes described for the $\mathrm{SCN}$ pacemaker, but clock phase is delayed by a couple of hours. In the hepatocyte, the clock components CLOCK and BMAL1 show highest activity around the day/night transition in both diurnal and nocturnal species indicating that the interplay between liver clock function and metabolic regulation must differ between animals with different activity profiles. It is assumed that liver clocks are reset by the $\mathrm{SCN}$ in a similar way as has been proposed for the adrenal. Again, like for the adrenal, between 5 and $10 \%$ of the liver transcriptome follows a circadian expression rhythm. Interestingly, liverspecific genetic disruption of clock function abrogates most, but not all of these rhythms, indicating that systemic cues exert a strong influence on liver physiology (Kornmann et al., 2007). The communication pathways between the SCN and the liver clock are largely unknown. Of note, adrenal-derived hormones such as GCs and norepinephrine can also affect the liver clock, which offers the possibility of an indirect entrainment pathway. Light appears capable of directly modulating clock gene expression in the liver (Cailotto et al., 2005). However, unlike what has been shown for the adrenal, the physiological implications of this process remain obscure.

\section{Local control of energy metabolism}

Insight on the effects of local liver clock function on metabolic processes comes mostly from studies on tissue-specific mouse mutants. Lamia and co-workers have shown that deleting the clock gene Bmall in hepatocytes using the Cre/loxP gene targeting system affects glucose metabolism, but has little overall effect on energy homeostasis (Lamia et al., 2008). LiverBmall mutant mice show hypoglycemia during the rest phase, exaggerated glucose clearance after insulin injection combined with a loss of the rhythmic expression of genes involved in glucose transport and the regulation of glucose utilization. The authors conclude that the liver clock may be important for buffering circulating glucose in a time-of-day dependent manner, thereby ensuring a constant supply of glucose as fuel for other organs such as the brain over the course of the day, while at the same time preventing the adverse effects of excessive blood glucose concentrations. Cho and colleagues have generated mice with liver specific mutations of two other clock components, Rev-erb $\alpha$ and Rev-erb $\beta$ (Cho et 
al., 2012). Double mutants show clock dysfunction in hepatocytes together with deregulated lipid metabolism. Microarray analyses reveal enrichment for changes in transcripts involved in insulin signaling, amino acid and lipid metabolism in the livers of the double mutants, correlating with increased circulating glucose and triglyceride levels, and a reduction in the level of free fatty acids observed in mice with an inducible (global) deletion of Rev$e r b \alpha / \beta$. Given that systemic and local (clock) controlled signals interact closely in the regulation of liver physiology more studies are needed to distinguish the function of local hepatocyte clocks from that of external regulatory factors.

\section{Local control of biotransformation}

The term biotransformation comprises metabolic processes associated with the conversion of biologically active substances (e.g. steroid hormones) and the modification and elimination of xenobiotics (such as drugs or toxins). In many cases the main goal of biotransformation is the polarization of lipophilic substances to increase their solubility and facilitate excretion via urine or bile. Detoxification can be divided into two major steps: modification and conjugation/excretion. Modification involves oxidatory reactions facilitated by the cytochrome P450 family of oxidases. Many P450 transcripts are controlled by the local clock machinery or by clock-controlled transcription factors such as the PARdomain basic leucine zipper transcription factors DBP, TEF, and HLF (Gachon et al., 2006). P450 activity is high during the active phase. Conjugation involves esterification of metabolites with polar species such as glutathione, glycine, and sulfuric or glucuronic acid by broad specificity transferases. Hepatic glutathione-Stransferase and glutathione peroxidase activities are rhythmic in mice, indicating a role for the circadian clock in the regulation of transfer reactions. Transport of modified metabolites into the blood or bile, again, is regulated in a circadian way, but it remains to be shown to which extent local clocks are involved in these processes (Levi and Schibler, 2007).

\section{Conclusion}

In multicellular organisms circadian timekeeping is organized in a complex hierarchical system with a central oscillator in the SCN and peripheral clocks in most tissues of the body. Adrenal and liver tissue clocks have been shown to impinge on the regulation of endocrine and physiological functions including diurnal rhythms of glucocorticoid secretion and energy metabolism. While the $\mathrm{SCN}$ is entrained primarily by the light/dark cycle, peripheral clocks respond to different external stimuli such as food intake, emphasizing the tight interaction between peripheral clock function and metabolism and allowing for a high degree of plasticity in the entrainment of endogenous timers to the external environment. The good accessibility of peripheral clocks makes them prime targets for chronopharmacological approaches. On the other hand, assessment of peripheral clock phase may become an important tool for determining internal circadian phase and tailor medication in the clinical practice. 


\section{Acknowledgements}

H.O. is an Emmy Noether fellow of the German Research Foundation (DFG) and a Lichtenberg fellow of the Volkswagen Foundation.

\section{References}

Andrews, R.V., and Folk, G.E., Jr. (1964). Circadian Metabolic Patterns in Cultured Hamster Adrenal Glands. Comparative biochemistry and physiology 11, 393-409.

Angeles-Castellanos, M., Amaya, J.M., SalgadoDelgado, R., Buijs, R.M., and Escobar, C. (2011). Scheduled food hastens re-entrainment more than melatonin does after a 6 -h phase advance of the lightdark cycle in rats. Journal of biological rhythms 26 , 324-334.

Bader, M. (2010). Tissue renin-angiotensinaldosterone systems: Targets for pharmacological therapy. Annual review of pharmacology and toxicology 50, 439-465.

Buijs, R.M., Kalsbeek, A., van der Woude, T.P., van Heerikhuize, J.J., and Shinn, S. (1993). Suprachiasmatic nucleus lesion increases corticosterone secretion. Am J Physiol 264, R11861192.

Cailotto, C., La Fleur, S.E., Van Heijningen, C., Wortel, J., Kalsbeek, A., Feenstra, M., Pevet, P., and Buijs, R.M. (2005). The suprachiasmatic nucleus controls the daily variation of plasma glucose via the autonomic output to the liver: are the clock genes involved? Eur J Neurosci 22, 2531-2540.

Cho, H., Zhao, X., Hatori, M., Yu, R.T., Barish, G.D., Lam, M.T., Chong, L.W., DiTacchio, L., Atkins, A.R., Glass, C.K., et al. (2012). Regulation of circadian behaviour and metabolism by REV-ERBalpha and REV-ERB-beta. Nature 485, 123-127.

Dallman, M.F., Engeland, W.C., Rose, J.C., Wilkinson, C.W., Shinsako, J., and Siedenburg, F. (1978). Nycthemeral rhythm in adrenal responsiveness to ACTH. The American journal of physiology 235, R210-218.

Dibner, C., Schibler, U., and Albrecht, U. (2010). The mammalian circadian timing system: organization and coordination of central and peripheral clocks. Annu Rev Physiol 72, 517-549.

Doi, M., Takahashi, Y., Komatsu, R., Yamazaki, F., Yamada, H., Haraguchi, S., Emoto, N., Okuno, Y., Tsujimoto, G., Kanematsu, A., et al. (2010). Saltsensitive hypertension in circadian clock-deficient Cry-null mice involves dysregulated adrenal Hsd3b6. Nature medicine 16, 67-74.

Gachon, F., Olela, F.F., Schaad, O., Descombes, P., and Schibler, U. (2006). The circadian PAR-domain basic leucine zipper transcription factors DBP, TEF, and HLF modulate basal and inducible xenobiotic detoxification. Cell metabolism 4, 25-36.

Guo, H., Brewer, J.M., Champhekar, A., Harris, R.B., and Bittman, E.L. (2005). Differential control of peripheral circadian rhythms by suprachiasmaticdependent neural signals. Proceedings of the National Academy of Sciences of the United States of America 102, 3111-3116.

Havelock, J.C., Auchus, R.J., and Rainey, W.E. (2004). The rise in adrenal androgen biosynthesis: adrenarche. Semin Reprod Med 22, 337-347.

Ishida, A., Mutoh, T., Ueyama, T., Bando, H., Masubuchi, S., Nakahara, D., Tsujimoto, G., and Okamura, H. (2005). Light activates the adrenal gland: timing of gene expression and glucocorticoid release. Cell Metab 2, 297-307.

Janssen, B.J., Tyssen, C.M., Duindam, H., and Rietveld, W.J. (1994). Suprachiasmatic lesions eliminate $24-\mathrm{h}$ blood pressure variability in rats. Physiol Behav 55, 307-311.

Kalsbeek, A., Ruiter, M., La Fleur, S.E., Van Heijningen, C., and Buijs, R.M. (2003). The diurnal modulation of hormonal responses in the rat varies with different stimuli. Journal of neuroendocrinology 15, 1144-1155. 
Kiessling, S., Eichele, G., and Oster, H. (2010). Adrenal glucocorticoids have a key role in circadian resynchronization in a mouse model of jet lag. J Clin Invest 120, 2600-2609.

Ko, C.H., and Takahashi, J.S. (2006). Molecular components of the mammalian circadian clock. Hum Mol Genet 15 Suppl 2, R271-277.

Kohsaka, A., and Bass, J. (2007). A sense of time: how molecular clocks organize metabolism. Trends Endocrinol Metab 18, 4-11.

Kornmann, B., Schaad, O., Bujard, H., Takahashi, J.S., and Schibler, U. (2007). System-driven and oscillator-dependent circadian transcription in mice with a conditionally active liver clock. PLoS Biol 5, e34.

Lamia, K.A., Storch, K.F., and Weitz, C.J. (2008). Physiological significance of a peripheral tissue circadian clock. Proc Natl Acad Sci U S A 105, 15172-15177.

Lemos, D.R., Downs, J.L., and Urbanski, H.F. (2006). Twenty-four-hour rhythmic gene expression in the rhesus macaque adrenal gland. Molecular endocrinology 20, 1164-1176.

Leproult, R., and Van Cauter, E. (2010). Role of sleep and sleep loss in hormonal release and metabolism. Endocrine development 17, 11-21.

Levi, F., Filipski, E., Iurisci, I., Li, X.M., and Innominato, P. (2007). Cross-talks between circadian timing system and cell division cycle determine cancer biology and therapeutics. Cold Spring Harb Symp Quant Biol 72, 465-475.

Levi, F., and Schibler, U. (2007). Circadian rhythms: mechanisms and therapeutic implications. Annu Rev Pharmacol Toxicol 47, 593-628.

Linsell, C.R., Lightman, S.L., Mullen, P.E., Brown, M.J., and Causon, R.C. (1985). Circadian rhythms of epinephrine and norepinephrine in man. The Journal of clinical endocrinology and metabolism 60, 12101215.

Oster, H., Damerow, S., Hut, R.A., and Eichele, G. (2006a). Transcriptional Profiling in the Adrenal
Gland Reveals Circadian Regulation of Hormone Biosynthesis Genes and Nucleosome Assembly Genes. J Biol Rhythms 21, 350-361.

Oster, H., Damerow, S., Kiessling, S., Jakubcakova, V., Abraham, D., Tian, J., Hoffmann, M.W., and Eichele, G. (2006b). The circadian rhythm of glucocorticoids is regulated by a gating mechanism residing in the adrenal cortical clock. Cell Metab 4, 163-173.

Son, G.H., Chung, S., Choe, H.K., Kim, H.D., Baik, S.M., Lee, H., Lee, H.W., Choi, S., Sun, W., Kim, H., et al. (2008). Adrenal peripheral clock controls the autonomous circadian rhythm of glucocorticoid by causing rhythmic steroid production. Proc Natl Acad Sci U S A 105, 20970-20975.

Stephan, F., and Zucker, H. (1972). Circadian rhythms in drinking behavior and locomotor activity of rats are eliminated by hypothalamic lesions. Proc Natl Acad Sci U S A 69, 1583-1586.

Ulrich-Lai, Y.M., and Herman, J.P. (2009). Neural regulation of endocrine and autonomic stress responses. Nature reviews Neuroscience 10, 397-409.

Vollmers, C., Gill, S., DiTacchio, L., Pulivarthy, S.R., Le, H.D., and Panda, S. (2009). Time of feeding and the intrinsic circadian clock drive rhythms in hepatic gene expression. Proceedings of the National Academy of Sciences of the United States of America 106, 21453-21458.

Waite, E.J., McKenna, M., Kershaw, Y., Walker, J.J., Cho, K., Piggins, H.D., and Lightman, S.L. (2012). Ultradian corticosterone secretion is maintained in the absence of circadian cues. The European journal of neuroscience.

Yoo, S.H., Yamazaki, S., Lowrey, P.L., Shimomura, K., Ko, C.H., Buhr, E.D., Siepka, S.M., Hong, H.K., Oh, W.J., Yoo, O.J., et al. (2004). PERIOD2::LUCIFERASE real-time reporting of circadian dynamics reveals persistent circadian oscillations in mouse peripheral tissues. Proc Natl Acad Sci U S A 101, 5339-5346.

Zhang, E.E., and Kay, S.A. (2010). Clocks not winding down: unravelling circadian networks. Nature reviews Molecular cell biology 11, 764-776. 


\subsection{Aims of the work}

The main aim of this work was to elucidate the physiological significance of the circadian clock in the GC-producing adrenocortical cells. For this purpose I used two genetic mouse models, with general and conditional deletions of the circadian clock. A deletion of the clock gene Bmall (Arntl) is sufficient to disrupt the functioning of the molecular circadian clockwork and this approach has been successfully used in other studies to evaluate functional roles of the clock in various tissues.

In the first part of the study, I characterized the functional state of the adrenocortical clock in Bmalldeficient male mice by investigating the capacity of the adrenal gland for rhythmic GC production under basal and stressed conditions. I hypothesized that a compromised clock function will lead to impaired GC biosynthesis in the adrenal gland, which would affect GC-related functions, especially in the response to stress. In support of this hypothesis, it has been previously reported that in another mouse model of clock deficiency, Per2/Cryl mice, the circadian rhythms of basal GC synthesis are impaired. However, in Per2/Cryl mice the negative limb of the circadian feedback mechanism is affected, whereas Bmall depletion targets a transcriptional activator of the positive limb of the circadian clock. Moreover, stress responses in circadian clock-deficient mice have so far been poorly investigated.

In the second part, I aimed at generating a conditional deficiency of the circadian clock in the adrenal cortex using the Cre-loxP gene targeting system. This would allow to better distinguish local from systemic effects of the circadian clock on adrenal gland function in vivo. My hypothesis was that the adrenocortical clock plays a role in the stabilization of circadian GC rhythms against external perturbation and can also be important during acute responses to stress. 


\section{Chapter 2. Results}

2.1. Publication „Impaired Glucocorticoid Production and Response to Stress in Arntl-Deficient Male Mice“

Alexei Leliavski, Anton Shostak, Jana Husse and Henrik Oster

Endocrinology, published online 4 Nov, 2013 


\title{
Impaired Glucocorticoid Production and Response to Stress in ArntI-Deficient Male Mice
}

\author{
Alexei Leliavski, Anton Shostak, Jana Husse, and Henrik Oster
}

\begin{abstract}
Chronophysiology Group (A.L., H.O.), Medical Department I, University of Lübeck, Lübeck, Germany; and Circadian Rhythms Group (A.L., A.S., J.H., H.O.), Max Planck Institute for Biophysical Chemistry, Gottingen, Germany
\end{abstract}

\begin{abstract}
The basic helix-loop-helix transcription factor ARNTL (also known as BMAL1 or MOP3) is a core component of the circadian timing system in mammals, which orchestrates 24-hour rhythms of physiology and behavior. Genetic ablation of Arntl in mice leads to behavioral and physiological arrhythmicity, including loss of circadian baseline regulation of glucocorticoids (GCs). GCs are important downstream regulators of circadian tissue clocks and have essential functions in the physiological adaptation to stress. The role of the clock machinery in the regulation of stressinduced GC release, however, is not well understood. Here we show that already under unstressed conditions Arntl-deficient mice suffer from hypocortisolism with impaired adrenal responsiveness to ACTH and down-regulated transcription of genes involved in cholesterol transport in adrenocortical cells. Under stress they show diminished GC and behavioral responses and develop behavioral resistance to acute and subchronic stressors, as shown using forced swim, tail suspension, and sucrose preference tests. These data suggest that the clock gene Arnt/ regulates circadian and acute secretion of GCs by the adrenal gland. Arnt/ disruption, probably via its effect on adrenal clock function, modulates stress axis activity and, thus, may promote resistance to both acute and repeated stress.
\end{abstract}

G lucocorticoid (GC) hormones play an essential role in the orchestration of physiology and behavior in response to stress (1-3), while at the same time GCs have been implicated in the entrainment of circadian rhythms $(4,5)$. Excessive GC production is associated with a variety of pathologies including metabolic deregulation and mood disorders such as depression and anxiety $(2,3)$. GCs, mainly cortisol in humans and corticosterone (CORT) in rodents, are predominantly produced by the adrenal glands in a pulsatile fashion with an underlying circadian rhythm (6). Adrenal GC secretion reflects the activation of the hypothalamic-pituitary-adrenal (HPA) axis. Hypophyseal ACTH binds to melanocortin-2 receptors (MC2Rs) in adrenocortical cells where it stimulates transport of cholesterol into mitochondria where CORT biosynthesis takes place. Daily GC peak levels are observed in the beginning of the activity phase, ie, in the early morning in humans and in the evening in nocturnal animals. Different mechanisms are involved in the circadian regulation of GC rhythms (reviewed in Refs. 6 and 7)

ISSN Print 0013-7227 ISSN Online 1945-7170

Printed in U.S.A.

Copyright 02013 by The Endocrine Society

Received June 5, 2013. Accepted October 23,2013. including molecular circadian clocks located in neurons of the hypothalamic suprachiasmatic nuclei (8) and in adrenocortical cells (9-11).

At the molecular level, these clocks are composed of transcriptional-translational feedback loops $(12,13)$, in which the transcription factors CLOCK and ARNTL activate Per and Cry genes, the products of which feed back on their own transcription by inhibiting CLOCK/ ARNTL. In addition, the CLOCK/ARNTL complex induces rhythmic transcription of a plethora of other genes, translating the activity of the molecular oscillator to rhythmic physiology. ARNTL plays a key role in the circadian clockwork, because, in mice, its deficiency leads to abrogation of endogenous behavioral and molecular rhythms $(14,15)$. GCs have been implicated in synchronizing circadian clocks in peripheral tissues and in the central nervous system (CNS) $(16,17)$. Activated GC receptors induce transcription of Per genes (18) or directly interact with clock proteins $(19,20)$.

Abbreviations: CORT, corticosterone; CT, circadian time; DD, constant dark; FST, forced swim test; GC, glucocorticoid; GR, glucocorticoid receptor; HPA axis, hypothalamic-pituitary-adrenal axis; LD, light-dark; SPT, sucrose preference test; TST, tail suspension test; ZT, Zeitgeber time. 
Stress-induced HPA axis activation and subsequent GC release mediate behavioral and physiological adaptation to various threatening conditions. Elevated blood GC levels stimulate the mobilization of energy substrates from liver and adipose stores, enhance memory formation, and promote effective coping with stress $(1,21)$. On the other hand, chronic stress/HPA axis activation may lead to various behavioral pathologies in mice and humans such as impaired memory and cognition, vulnerability to depression and anxiety, and abnormal reward seeking (2, 22, 23).

In the present study we focus on how Arntl regulates GC release from the adrenal gland. We find impaired CORT production and ACTH responses in Arntl-deficient mice together with blunted behavioral effects of acute and subchronic stress, suggesting a critical role of the circadian clock gene Arntl in the physiological adaptation to stress.

\section{Materials and Methods}

\section{Animals and housing}

All animal experiments were ethically approved and licensed by the local state authorities and executed according to the regulations of the German Animal Welfare Act (TierSchG). Male wild-type and $\mathrm{Arntl}^{-1-}$ mice (3-4 months old ) on a C57BL6J background were individually housed under 12 -hour light, 12 hour dark conditions (LD; 300-400 lux) with ad libitum access to food and water. For adrenal slice culture experiments and analysis of gene expression in constant darkness conditions (DD), single-housed animals were LD-entrained for at least 1 week and then released into DD. Tissues were collected 36 and 48 hours after "lights off," which roughly corresponds to circadian times (CT) 0 and CT12, ie, the beginning and end of the animal's rest phase, in wild-type animals. For hormonal measurements, blood samples were also collected at CT6 and CT18 (42 and 54 hours after lights off).

\section{Behavioral tests}

\section{Forced swim test (FST)}

The test was performed at the end of the light phase (between Zeitgeber time (ZT) 10 and ZT12, ie, 10-12 hours after "lights on") as described in Ref. 24, with minor modifications. Briefly, animals were placed for 6 minutes into a standard 3-L glass beaker filled with tap water $\left(25 \pm 2^{\circ} \mathrm{C}\right)$ from which they could not escape. Every session was video recorded, and the duration of immobility over every minute of the 6-minute test was estimated using the CowLog open source software (http://cowlog.org) (25).

\section{Repeated restraint stress}

Mice were exposed to confinement stress once daily for 2 hours between ZT10 and ZT12 for 7 consecutive days (26) by keeping them in small transparent plastic restrainers $(95 \times 30 \times$ $32 \mathrm{~mm}$ ). Sucrose preference tests (SPTs; see below) were con- ducted 1 day before the restraint period (baseline sucrose preference, see below) and after 7 restraint sessions. Immobility behavior during a tail suspension test (TST; see below) was assessed twice, 1 day before the beginning of the first and 1 day after the last restraint session.

\section{TST}

TSTs were conducted according to a protocol described elsewhere (24). Between ZT10 and ZT12, mice were suspended for 6 minutes by the tail on a horizontal bar at a height of $20-25 \mathrm{~cm}$. Every session was video recorded in the absence of the experimenter. The duration of immobility (ie, passive hanging without movements) over the course of the 6-minute test was measured with assistance of the CowLog software.

\section{$S P T$}

To estimate baseline sucrose preference (27), mice were provided a choice between 2 bottles filled with $1 \%$ sucrose solution and tap water. To avoid positional preference, bottle positions were changed twice a day, in the middle of activity and rest phases, respectively. The bottles were weighed once a day (at the end of light phase) for 3 consecutive days, and sucrose and water intake were averaged. Sucrose preference after restraint stress was evaluated over a 24 -hour period. To calculate the percentage of sucrose preference, the amount of consumed sucrose solution was divided by the amount of total liquid intake and multiplied by 100 .

\section{Quantitative RT-PCR}

Quantitative analysis of mRNA levels was performed as described elsewhere (28). Total RNA was extracted from whole adrenal tissues using TRIzol Reagent (Life Technologies) according to the manufacturer's instructions. cDNA was synthesized using High Capacity cDNA Reverse Transcription kit (Life Technologies). Quantitative real-time PCR was performed on a C1000 Thermal Cycler and CFX96 Real-Time PCR Detection System (Bio-Rad Laboratories) with GoTaq qPCR Master Mix (Promega Corp.), and relative expression was assessed by comparison with Eef1a1 using the $\Delta \Delta C T$ method (9). Primer sequences are listed in Supplemental Table 1 published on The Endocrine Society's Journals Online web site at http://endo.endojournals.org.

\section{Adrenal responsiveness to ACTH ex vivo}

Adrenal slice culture and adrenal ACTH stimulation ex vivo were performed as described previously (10). Briefly, 200- $\mu \mathrm{m}$ slices were precultured for 20 minutes on Millicell-CM membranes (Millipore Corp.) in DMEM (PAA Laboratories) supplemented with $0.1 \%$ dimethyl sulfoxide, $50 \mu \mathrm{M} 2$-mercaptoethanol, $2 \%$ fetal bovine serum, and $0.12 \mathrm{mg} / \mathrm{mL}$ penicillin/ streptomycin at $37^{\circ} \mathrm{C}$ and $5 \% \mathrm{CO}_{2}$. Slices were stimulated with $20 \mathrm{nM}$ ACTH and medium was collected immediately $(0 \mathrm{~min}-$ utes), 30 minutes, 90 minutes, and 210 minutes later. To study dose response to $\mathrm{ACTH}$, adrenal slices were stimulated with 0 , 2 , and $20 \mathrm{nM} \mathrm{ACTH}$, and medium was collected 90 minutes later. Samples were stored at $-80^{\circ} \mathrm{C}$ until further processing.

\section{Dexamethasone suppression test}

Mice received ip injection of dexamethasone solution $(100 \mu \mathrm{g}$ per $\mathrm{kg}$ of body weight in $0.9 \%$ saline) at ZT 8 , and trunk blood 
was collected 6 hours later for corticosterone (CORT) analysis, as described below. A control group was injected with $200 \mu \mathrm{L}$ of $0.9 \%$ saline solution. The selected dosage was previously shown to be efficient in suppressing CORT production down to about $20 \%$ compared with saline-injected controls (29).

\section{Sample preparation and hormone measurements}

Animals were removed from their cages and immediately culled by cervical dislocation. Trunk blood was collected in $\mathrm{Mi}$ crovette 300 EDTA-coated tubes (Sarstedt), centrifuged at $2200 \times g$ for 20 minutes at $4^{\circ} \mathrm{C}$, and plasma was frozen at $-80^{\circ} \mathrm{C}$ until use. The time passed between opening the cage and finishing blood collection was usually around 1 minute, but never more than 2 minutes. Fecal samples were collected at 4 -hour intervals and stored at $-80^{\circ} \mathrm{C}$ until extraction. Fecal corticoid extraction was done according to a previously published protocol (30). $\mathrm{CORT} /$ corticoid concentrations were measured using a commercially available RIA kit from MP Biomedicals (catalog no. 07120103). Plasma samples were diluted at 1:200, fecal extracts at $1: 5$, and medium samples at $1: 10$, respectively. ACTH plasma concentrations were analyzed using the IMMULITE $1000 \mathrm{Im}$ munoassay System (Siemens) at 1:2 to 1:4 dilutions.

\section{Histologic analysis}

Isolated adrenal glands were removed from surrounding fat, weighed, fixed in $4 \%$ paraformaldehyde, and embedded in paraffin. Adrenal sections $(8-\mu \mathrm{m})$ were stained with hematoxylineosin. To evaluate adrenal cortex-to-medulla ratio, cortical and
A

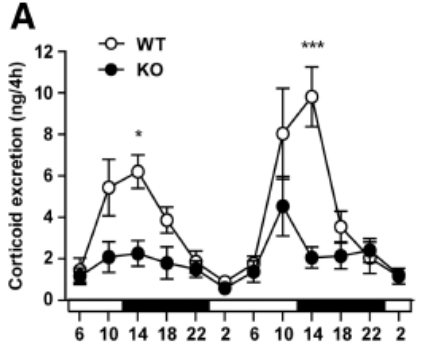
Zeitgeber time (hours)

D

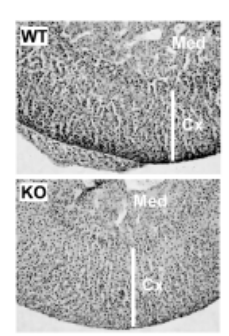

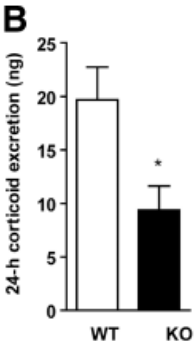

WT ko

E

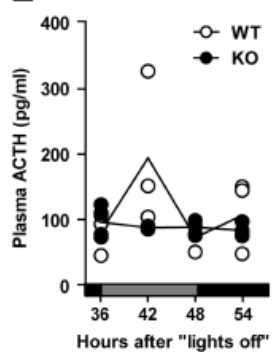

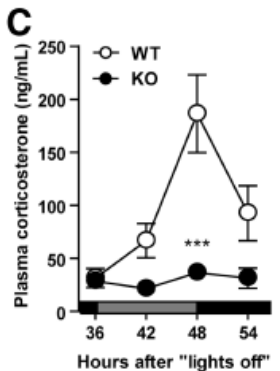

$\mathbf{F}$

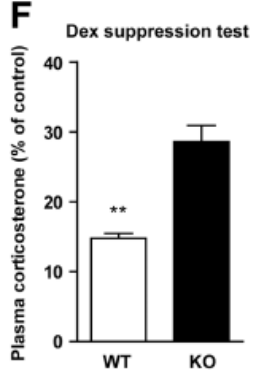

Figure 1. Hypocortisolism in Arntl ${ }^{-1-}$ mice $A$ and B, Profile (48 hour) of corticoid excretion (A) and total amount of excreted corticoids per day (B) in fecal samples from wild-type (WT) and Arnt $^{-1-}$ (knockout [KO]) mice kept in LD $(n=5-6)$. C and D, Plasma CORT (C) and plasma ACTH (E) levels in WT and KO mice on the second day in DD $(n=3-5)$. D, Hematoxylin and eosin staining of WT and KO adrenals (Cx, adrenal cortex; Med, medulla); magnification, 20×. F, Dexamethasone (Dex) suppression test. Suppressive effect of dexamethasone on CORT production is normalized to CORT levels of saline-injected control mice $(n=4)$. ${ }^{*}, P<.05$; $*$ * $P<.01 ;{ }^{* \star}, P<.001$ (two-way ANOVA with Bonferroni post hoc test [panels $\mathrm{A}, \mathrm{C}$, and $\mathrm{E}$; Student's $t$ test $[\mathrm{B}$ and $\mathrm{F}]$ ). medullar areas were measured from every specimen in at least 3 different sections close to the middle of the adrenal gland. Lipid treated or repeatedly stressed mice (3 sequential 10-minute sessions with 30-minute rest intervals). Cryosections $(10-\mu \mathrm{m})$ were rinsed in $60 \%$ isopropanol and stained with solution (Sigma-Aldrich) for 10 minutes. Image analysis was performed with Image J software (National Institutes of

\section{Statistical analysis}

Statistical analysis was performed using GraphPad Prism 5.0 GraphPad). All data are represented as means \pm SEM. Whenever applicable, normality of data distribution was confirmed sizes outlier tests were performed, revealing no indications of al distribution. Two-group comparisons were done using unpaired $t$ tests. For multiple comparisons one- or two-way the figure legends. Time course analyses (Figures 1, A, C, and E, and Supplemental Figure 1) were performed usin

\section{Results}

\section{Hypocortisolism in $\mathrm{Arntl}^{-/-}$} mice

To test whether Arntl deficiency affects daily dynamics of GC production, we measured corticoid excretion in feces of $\mathrm{Arntl}^{-1-}$ and congenic wild-type mice at 4-hour intervals over the course of 2 days (30). Fecal corticoid excretion profiles have been shown to faithfully mimic blood CORT levels, with a delay of 4-6 hours, while allowing for repeated noninvasive sampling from individual animals and reducing variability caused by ultradian CORT oscillations $(30,31)$. As expected, corticoid excretion in Arntldeficient mice showed strongly dampened diurnal rhythmicity (Figure 1A). Moreover, overall corticoid excretion in mutants was reduced by about $50 \%$ in comparison with agematched wild-type controls (Figure 1B). Similarly, nonrhythmic and overall low CORT levels were observed in plasma and fecal samples of $\mathrm{Arntl}^{-/-}$mice kept in DD (Figure 1C and Supplemental Figure 1). 
Histologic examination of Arntl-deficient adrenals did not reveal any gross defects in adrenal morphology (Figure 1D and Supplemental Figure 2B). Relative adrenal weight was even slightly increased in $\mathrm{Arntl}^{-1-}$ animals compared with wild-type controls (Supplemental Figure 2A). Hypocortisolism could result from deregulation of the HPA axis upstream of the adrenal gland, eg, by diminished ACTH synthesis or release from the pituitary (32). To address this point, we measured plasma ACTH levels in wild-type and Arntl-deficient mice at 4 different times on the second day in DD. We found no significant differences in ACTH concentrations between wild-type and $\mathrm{Arntl}^{-1-}$ mice (Figure $1 \mathrm{E})$ at any of the time points examined, although individual variation at 42 hours in wild-type mice was quite high, suggesting that reduced CORT production may not simply represent the result of reduced ACTH signal. The combination of largely unchanged ACTH levels with a hypocortisolic state in $\mathrm{Arntl}^{-1-}$ mice may indicate blunted sensitivity of the HPA axis to negative CORT feedback. Indeed, we found that dexamethasone was less effective in inhibiting CORT production in knockout mice (Figure 1F).

\section{ACTH hyposensitivity in $\mathrm{Arntl}^{-/-}$adrenal slice culture}

These findings let us to hypothesize that hypocortisolism in $\mathrm{Arntl}^{-1-}$ mice may result, at least in part, from reduced sensitivity of the adrenal cortex to ACTH stimulation. To test this, we cultured adrenal tissue slices from wild-type and $\mathrm{Arntl} \mathrm{I}^{-1-}$ mice culled at 36 and 48 hours after lights off and stimulated them with $20 \mathrm{nM} \mathrm{ACTH}$ to measure CORT responses ex vivo. In wild-type explants CORT production was rapidly induced upon ACTH stimulation at both time points, with higher responsiveness at 48 hours correlating with high in vivo CORT levels at this time point (10) (Figure 2A). In accordance with our hypothesis, the ability of $\mathrm{Arntl}^{-/-}$adrenals to respond to ACTH stimulation did not differ between the 2 time points and was dramatically reduced compared with wild types (Figure 2B). A dose response determined at 48 hours confirmed the reduced CORT response of $\mathrm{Arntl}^{-/-}$adrenal slices to ACTH concentrations at various concentrations (Figure 2C).

\section{$\mathrm{Arntl}^{-/-}$mice show decreased CORT and behavioral responses to acute stress}

The altered CORT-to-ACTH ratio under undisturbed conditions in vivo, together with the blunted ACTH sensitivity of adrenal slices, suggested that Arntl deficiency may also lead to altered CORT responses after acute stress. To test this, wild-type and $\mathrm{Arntl}^{-/-}$mice were subjected to an FST as an acute stressor (33), and plasma
CORT levels were measured in different cohorts before and after the FST. Because differences in adrenal sensitivity to ACTH between wild-type and mutants were highest at 48 hours in DD (Figure 2), all further experiments were performed at this time point. As expected, in wild-type mice FST caused a more than 3-fold elevation of CORT levels compared with baseline conditions (Figure 3A). In contrast, no significant up-regulation of CORT levels, but preserved ACTH responses, were observed in stressed Arntl $^{-1-}$ mice (Figure 3, A and B). In line with this, wildtype mice showed increasing amounts of immobility over the first 6 minutes of the FST, which is usually interpreted as despair-like behavior (Figure 3C). In contrast, and analogous to their absent CORT response, Arntl $^{-1-}$ mice stayed invariably active during the whole course of the test (Figure 3C). Of note, body position in water during immobility bouts was unaltered in Arntl-deficient mice (Supplemental Figure 3), and the duration of immobility bouts in a repeated FST was comparable to those in wild-type counterparts (data not shown). This suggests that the observed vigorous swimming was not merely a behavioral adaptation of Arntl-deficient mice to a compromised ability of staying afloat due to unrelated physical and meta-

\section{A}
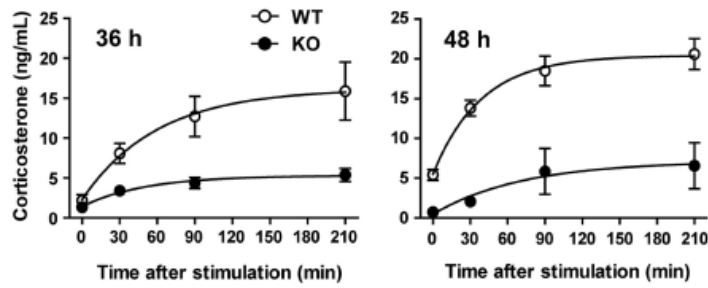

Time after stimulation $(\mathrm{min})$

B

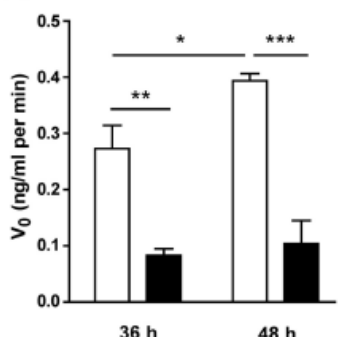

C

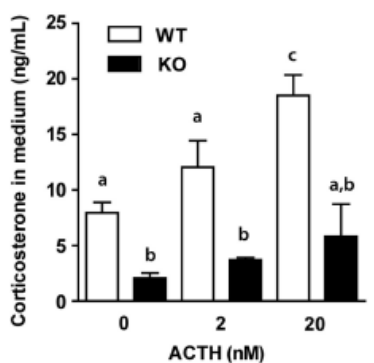

Figure 2. Reduced responsiveness of $\mathrm{Arnt}^{-1-}$ adrenal explants to ACTH stimulation A, Dynamics of CORT release into the medium after stimulation of wild-type (WT) and knockout (KO) adrenal slices with 20 $\mathrm{nM} \mathrm{ACTH}(\mathrm{n}=3-6)$ in adrenal slice explants prepared at 36 hours (left panel) or 48 hours (right panel) after lights off. B, Initial rate of CORT release from $\mathrm{ACTH}$-treated $\mathrm{WT}$ and $\mathrm{KO}$ adrenal explants. Two-way ANOVA revealed significant effect of genotype on the initial rate $(P<$ .0001). C, Dose response to ACTH stimulation in WT and KO adrenal slices. Identical letters indicate the absence of significant differences between columns. V0, Release rate at $\mathrm{t}=0 ;{ }^{*}, P<.05$; ${ }^{*}{ }^{\star}, P<.01$; $\star * *, P<.001$ (Bonferroni post hoc test) 
bolic abnormalities $(34,35)$. Reduced body temperature (Supplemental Figure 3C) and impaired muscle strength, as reported in Arntl mutants (34), would rather promote the time spent immobile in the FST (36), but the opposite phenotype was observed in $\mathrm{Arntl}^{-1-}$ mice. Together, we conclude that Arntl-deficient mice show HPA axis insensitivity correlating with behavioral resistance in response to acute stress evoked by forced swimming.

\section{Altered adrenal expression of transcripts involved in cholesterol transport and ACTH signaling}

To identify a potential mechanism underlying the observed changes in the regulation of CORT production in Arntl ${ }^{-1-}$ mice, we measured the mRNA levels of genes involved in adrenocortical physiology (summarized in Supplemental Figure 4) using quantitative RT-PCR. We hypothesized that potential ARNTL target genes, being under control of this essential transcription factor of the circadian clock, are likely to be expressed in a circadian manner (eg, Mc2r, Mrap, Prkce, Sp1, Nr5a1, NrOb1, Star, $L d l r$, Stard 4, Por), as has been reported in previous studies $(9,10,37)$. In addition, we included genes that encode key steroidogenic enzymes (Cyp11a1, Cyp11b1, Hsd3b1) and proteins involved in transport of cholesterol as the main substrate of CORT biosynthesis (Scarb1, Nr1b3). We found that the mRNA levels of most genes associated with adrenal development and steroidogenesis (Cyp11a1, Cyp11b1, Nr5a1, NrOb1, Nr1b3) remained largely unaltered in $\mathrm{Arntl}^{-1-}$ adrenal glands (Figure 4A). In contrast, several key genes involved in cholesterol trafficking (Star, $L d l r$, Stard4) were down-regulated by $50 \%$ or more in Arntl-deficient adrenals (Figure 4C), while, at the same time, the expression of $\mathrm{Mc} 2 r$, which encodes the ACTH receptor, appeared elevated in $\mathrm{Arntl}^{-/-}$compared with wild-type adrenals (Figure 4B).
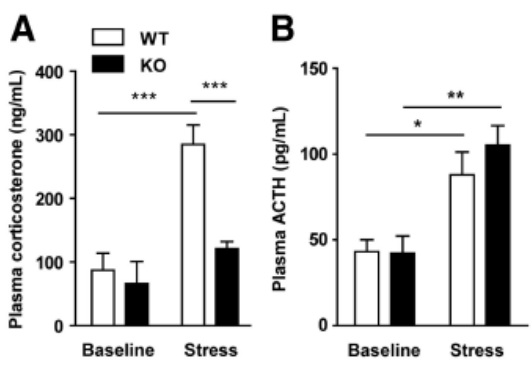

C

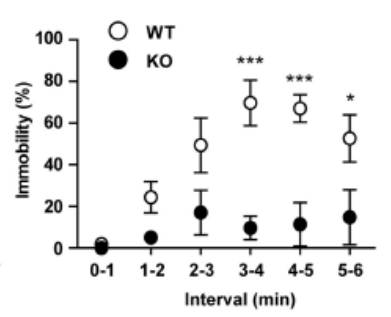

Figure 3. Reduced hormonal and behavioral responses to acute stress in $\mathrm{Arnt}^{-1-}$ mice $\mathrm{A}$ Plasma CORT and ACTH concentrations in wild-type (WT) and knockout (KO) animals before and after acute stress (forced swimming) at 48 hours after lights off ( $n=4-10)$. In panel A, two-way ANOVA revealed significant effects of genotype and treatment and interaction between both factors $(P=0.008 ; P=0.0007$; and $P=0.034$, respectively). In panel $\mathrm{B}$, a significant effect of treatment was found $(P=0.0003)$. $C$, Time course of immobility behavior (in percent) during 6 minutes of forced swimming in WT and KO mice $(n=4-5) .{ }^{*}, P<.05 ;{ }^{* *}, P<.001$ (Bonferroni post hoc test).
Translocation of cholesterol to the mitochondrion is the rate-limiting step of steroidogenesis and, therefore, down-regulation of the cholesterol transport machinery may explain the blunted CORT responsiveness to ACTH stimulation or stress observed in $\mathrm{Arntl}^{-1-}$ adrenals. To test this more directly, we analyzed adrenal lipid content in untreated and stressed mice using Oil Red O staining. In wild-type mice cholesterol esters that make up the vast majority of lipids stored in adrenocortical lipid droplets became depleted dramatically after repeated swimming stress. In the mutants baseline levels were already reduced compared with wild types, but importantly, little effect was observed after stress (Figure 4D), which would be in line with an incapacity to transport cholesterol into mitochondria for conversion into CORT.

To test whether altered levels of gene expression in $\mathrm{Arntl}^{-1-}$ adrenals may reflect a general metabolic deficiency, we analyzed levels of the regulated transcripts in different metabolic tissues. Whereas Por was similarly down-regulated in liver, adipose tissue, and muscle, differential effects on Ldlr, Star and Stard4 expression after deletion of Arntl were found in different tissues (Supplemental Figure 5). These data do not support the hypothesis that systemic metabolic changes in Arntl-deficient mice are responsible for the reduced expression of cholesterol transport genes.

In summary, our results suggest that deregulation of gene expression, particularly genes involved in cholesterol transport, in Arntl-deficient adrenal glands may contribute to compromised adrenal responsiveness to ACTH and, hence, reduced CORT response to stress.

\section{$\mathrm{Arntl}^{-1-}$ mice are resistant to behavioral changes induced by repeated restraint stress}

It is well accepted that chronic/prolonged stress induces dramatic changes in animal and human behavior, including increased susceptibility to depression, anxiety, and drug addiction $(1,2,23)$. We hypothesized that, complementary to reduced acute stress responses, behavioral changes in response to a subchronic stressor might also be altered in Arntl-deficient mice. To address this point we used a 1-week repeated restraint stress paradigm with SPTs and TSTs tests as behavioral outputs (38-40). Under baseline conditions sucrose preference as well as absolute intake of $1 \%$ sucrose solution were reduced in $\mathrm{Arntl}^{-/-}$mice compared with age-matched wild- 
type controls (Figure 5, A and B). After 1 week of daily restraint wild-type mice significantly increased sucrose intake and sucrose preference, whereas in $\mathrm{Arntl}^{-1-}$ mice, no change in sucrose intake behavior was observed (Figure 5, $\mathrm{A}$ and B). Moreover whereas wild-type mice showed increased immobility in the TST repeated after 1 week of constraint stress, Arntl-deficient animals were significantly less immobile at the end of the stress period (Figure $5 \mathrm{C})$. Taken together, we conclude that the circadian clock gene Arntl regulates behavioral responses to acute and chronic stressors, potentially via modulation of adrenal CORT secretion.

\section{Discussion}

In the current study we demonstrated that Arntl is necessary for normal CORT production and responses to acute and repeated stress. Arntl-deficient mice show hypocortisolism without changes in ACTH secretion. Arntl ${ }^{-/-}$adrenals are less sensitive to ACTH stimulation ex vivo correlating with reduced expression of cholesterol transport genes Star, Stard 4 , and Ldlr. Together these changes may lead to blunted GC and behavioral responses to stress.

We observed that $\mathrm{Arntl}^{-/-}$mice show low levels of plasma CORT and blunted circadian corticoid rhythms under LD and DD conditions (Figure 1 and Supplemental Figure 1), confirming previous findings that Arntl is indispensable for maintenance of physiological circadian rhythms. Of note, Rudic et al (41) did not observe a loss of daily GC variation in $\mathrm{Arntl}^{-/-}$mice in DD, but plasma CORT was measured only at 2 time points (CT4 and CT16), whereas the normal peak and trough of GC secretion (CT0 and CT12) were not assessed. Hypocortisolism has also been reported in mice carrying a mutation in the gene encoding for the ARNTL partner CLOCK (42), whereas, to the contrary, a lack of Cry genes results in up-regulated CORT levels $(20,28)$. This is consistent with the view that the components of the positive limb of the circadian clockwork, ARNTL and CLOCK, promote GC production, whereas members of the negative branch have opposite effects. Similarly, constant dis-inhibition of ARNTL/CLOCK activity in Cry $1 / 2$ double-mutant mice promotes overproduction of another adrenal corticoid, aldosterone (43). Blood CORT levels are low in Per 2 single- and Per $2 / C r y 1$ double-mutant mice $(10,44)$, possibly reflecting the positive impact of PER proteins on Arntl transcription (45).

Hypocortisolism, a main feature of adrenal insufficiency, can be caused by a variety of primary and secondary factors, including impaired HPA axis activity and steroidogenesis or GC metabolism, but also defects in adrenal development (46). Deletion of Arntl did not cause any significant alterations in ACTH levels. Together with reduced ACTH sensitivity (Figure 2), this suggests that blunted CORT secretion in Arntl ${ }^{-/-}$mice may be rooted in the adrenal itself, ie, representing a case of primary hypocortisolism. However, low GC levels were not mirrored by a dis-inhibition of ACTH release from the pituitary, which is in accordance with impaired sensitivity of the HPA axis to inhibitory CORT feedback (Figure 1, E and F). This could have developmental reasons or may simply reflect an additional effect of Arntl deficiency on GC feedback target regions, ie, the hypothalamus or the pituitary. In line with this, it was found that Arntl is required for induction of Per2 expression by GCs (19).

In addition to regulating circadian clock function, Arntl plays an important role in tissue development, eg, in skeletal muscle and adipose tissues $(34,47)$. However, our findings, together with published observations $(10,11)$, suggest that hypocortisolism in $\mathrm{Arntl}^{-/-}$mice seems to represent a functional defect, rather than a result of aberrant adrenal development. Indeed, analysis of $\mathrm{Arntl}^{-/-}$ adrenal weight, morphology, and cortex-to-medulla ratio did not indicate gross developmental abnormalities. Additionally, the expression of the key transcription factors SF1 (encoded by $\mathrm{Nr} 5 a 1$ ) and DEX1 (NrOb1) regulating adrenal gland development was not altered in Arntl-deficient adrenals (Figure 4).

Our data and previous studies suggest that circadian clock gene deficiency promotes adrenal ACTH resistance $(10,48)$. The clock machinery regulates cellular physiology via transcriptional programs (12). This lead us to hypothesize that hypocortisolism in $\mathrm{Arntl}^{-/-}$mice may be the consequence of altered expression of clock target genes involved in regulating the steroidogenesis $(9-11,37)$. Indeed, the down-regulation of cholesterol transport ( $L d l r$, Star, Stard4) and steroidogenic (Por) genes in Arntl-deficient adrenals, together with a lack of cholesterol depletion upon stress (Figure 4), may provide an explanation for the observed ACTH resistance and blunted GC synthesis. In line with our findings, both Por and Star have previously been shown to be directly clock-controlled genes $(11,49)$. Therefore, a similar reduction of the transcript levels in muscle and adipose tissue of $\mathrm{Arntl}^{-/-}$mice (Supplemental Figure 5) is not unexpected. To the contrary, the ACTH receptor gene $M c 2 r$ was up-regulated, an effect that is likely to be attributable to reduced GC feedback but might also be related to an indirect control of its expression by the circadian clock.

Impaired ACTH sensitivity may not only cause hypocortisolism but may also lead to compromised GC and behavioral responses to stress. We used the forced swimming paradigm as an acute, predominantly physical stres- 
sor (33). Consistent with reduced adrenal responsiveness to ACTH stimulation ex vivo, Arntl-deficient mice had dramatically blunted CORT, but not ACTH, responses to acute stress compared with wild-type controls (Figure 3). Both duration and type of stressor are critical for programming the intensity of evoked GC responses $(1,2)$. In line with this, a longer and more intense stressor (immobilization) is still able to induce GC responses in $\mathrm{Arntl}^{-/}$ mice $(50)$ or in mice with a compromised adrenal clock (11).
A

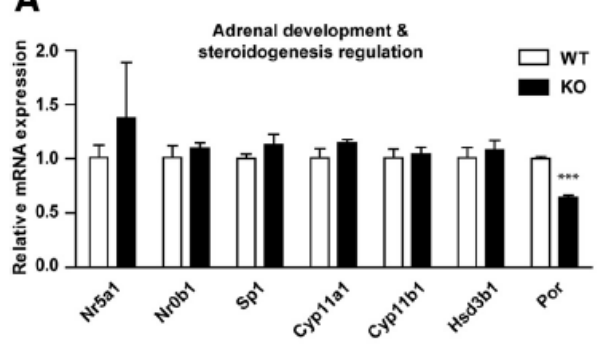

B
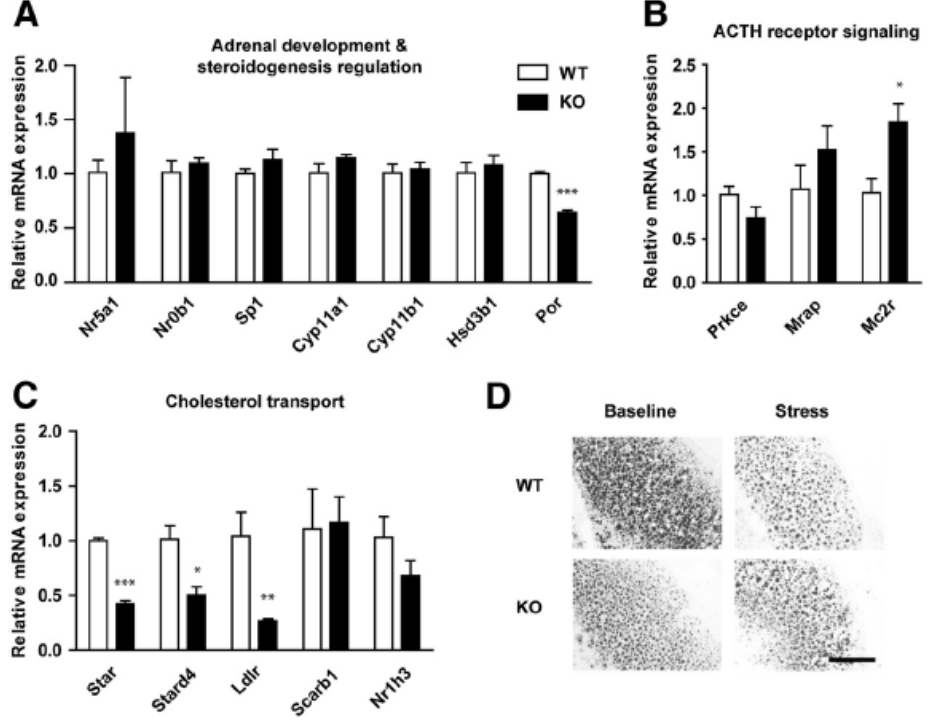

D

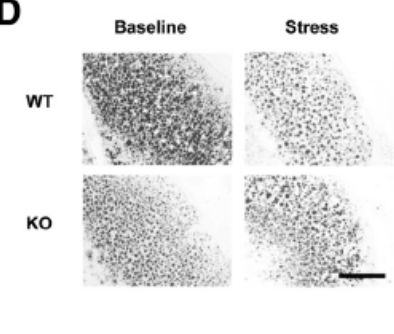

Figure 4. Arnt/-deficient mice show altered steroidogenesis-associated gene expression in the adrenal gland A-C, Comparison of mRNA levels of genes involved in adrenal function and steroid biosynthesis in adrenals from wild-type $(\mathrm{WT})$ and knockout $(\mathrm{KO})$ mice at 48 hours after lights off $(n=3-4)$. D, Oil Red O staining of adrenal sections from untreated and stressed WT and KO mice. Scale bar, $200 \mu \mathrm{m}$. ${ }^{*}, P<.05 ;{ }^{\star *}, P<.01 ;{ }^{* *}, P<.001$ (Student's $t$ test [panels $A-C])$.
A

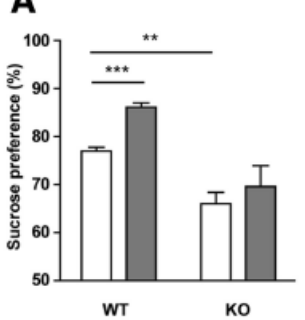

B

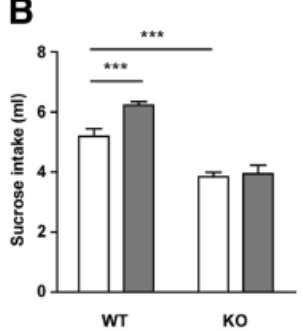

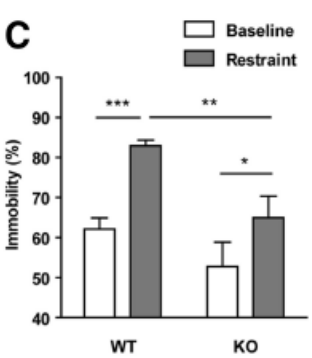

Figure 5. $\mathrm{Arnt}^{-1-}$ mice are resistant to behavioral changes induced by repeated restraint stress A and B, Sucrose preference (A) and sucrose consumption (B) in wild-type (WT) and knockout (KO) mice before and after 7 days of chronic restraint stress $(n=6-9)$. Effects of genotype and stress were significant for sucrose preference $(P<0.0001$ and $P=0.003$, respectively). Effects of genotype and stress and interaction between 2 factors were significant for sucrose consumption $(P<0.0001 ; P=0.002$; and $P=0.006$, respectively). C, TST immobility behavior (in percent) before and after repeated restraint measured in the same cohorts of mice as in panels $A$ and $B$. Effects of genotype and stress were significant $\left(P=.012\right.$ and $P<.0001$, respectively). ${ }^{*}{ }^{*}, P<$ $.01 ; * \star *, P<.001$ (repeated measures two-way ANOVA with Bonferroni posttest).
GCs exert a plethora of effects on animal behavior via binding to corticoid receptors in the brain (1). Clinical and experimental data support a key role of excessive GC production in the pathogenesis of depression (2). In contrast, chronically reduced GC levels may lead to opposite tion of CORT production in rodents by adrenalectomy or metyrapone treatment, as well as blockade of central GC effects by glucocorticoid receptor deletion in the brain, led to reduced immobility in the FST (51-53). The same test revealed a drastic reduction in immobility in $\mathrm{Arntl}^{-/-}$mice (Figure 3), indicating resistance to acute stress effects, despite the fact that Arntl deficiency causes development of progressive arthropathy and impairment of locomotor activity (35). Similarly, a tendency toward reduced immobility was seen in Arntl $^{-1-}$ mice during the TST, which became significant during repeated testing (Figure 5). Conversely, Arntldeficient animals were found to be anhedonic, reflected by a reduced baseline sucrose preference. This could be, in part, an effect of altered olfaction or taste in $\mathrm{Arntl}^{-1-}$ mice (54). It further implies that the mood phenotype of Arntl ${ }^{-/}$mice may vary depending on whether activation of the stress axis is involved. In other words, Arntl deficiency may not be protective for the development of depression but may confer resistance against the mood effects of stress. Detrimental effects of repeated stress are based on recruitment of neural pathways that are distinct from those involved in acute stress responses (reviewed in Ref. 3). We applied repeated restraint stress to reveal whether Arntl-deficient mice are also less sensitive to prolonged stress effects (55). Extensive chronic stress leads to signs of anhedonia in rodents such as reduced sucrose preference (39). To the contrary, a shorter, subchronic stress (up to 3 weeks) is usually associated with increased reward-seeking behavior $(39,56)$, which is interpreted as a compensation for stress-associated deficits in reward signaling (23). 
In line with this, wild-type mice responded to repeated restraint by a gradual increase in sucrose consumption and sucrose preference (Figure 5). In contrast, repeated restraint did not affect sucrose intake in Arntl-deficient mice, which is consistent with the view that GCs regulate the activation of mesolimbic reward circuits and dopamine release in the nucleus accumbens (57). Reduced immobility in the FST has previously been observed in Clock $^{\Delta 19}$ and Per $2^{\text {Brdm1 }}$ mutant mice $(58,59)$. In both cases, the phenotype has been contributed to by local clock gene effects in the brain. However, both strains also show decreased daily CORT production $(20,42)$, which together with our data suggests that peripheral clock regulation may also play a role in this context (see also Ref. 60). At the same time, and in line with our dexamethasone suppression data, it suggests that the observed behavioral resistance of $\mathrm{Arntl}^{-/-}$mice to stress may also be influenced by deregulated glucocorticoid receptor signaling in the brain $(19,20)$. Tissue-specific genetic targeting of the clock gene machinery will help to better clarify the contribution of different sites of action of Arntl in this context.

In conclusion, our data on adrenal CORT regulation in $\mathrm{A} r n t l^{-/-}$mice provide a complementary perspective on the regulation of stress responses and mood. It has been documented that disruption of the normal light-dark cycle in humans and rodents can lead to excessive HPA axis activation and symptoms of depression ( Ref. 27 and reviewed in Ref. 60). In contrast, we and others observed that a genetic disruption of the molecular clock in mice can also confer hormonal and behavioral resistance to stress. This effect may be mediated, at least in part, by regulation of adrenocortical clocks, thus potentially providing a new and easily accessible target for the treatment of stressassociated disorders.

\section{Acknowledgments}

We thank Dr. Johanna Barclay (University of Queensland, Queensland, Australia) for critical reading of the manuscript, Drs. Olaf Jöhren and Volker Ott and Ines Stölting (Institutes of Neuroendocrinology and Experimental and Clinical Pharmacology and Toxicology, University of Lübeck, Lübeck, Germany) for generous technical support, and all members of the Circadian Rhythms Group for helpful discussions.

Address all correspondence and requests for reprints to: Professor Dr. Henrik Oster, Medical Department I, University of Lübeck, Ratzeburger Allee 160, 23538 Lübeck, Germany, Email: henrik.oster@uksh.de.

This work was supported by the German Research Foundation (DFG), the Max Planck Society, and a Gerhard Hunsmann
Fellowship (to A.L.). H.O. is a Lichtenberg fellow of the Volkswagen Foundation.

Present addresses for B.Z.H. and A.S.: Circadian Rhythms and Molecular Clocks Group (B.Z.H.), and Biochemistry Center (A.S.), Heidelberg University, Heidelberg, Germany.

Present address for J.H.: Program in Neurosciences and Mental Health, The Hospital for Sick Children, Toronto, Canada.

Disclosure Summary: The authors have nothing to disclose.

\section{References}

1. de Kloet ER, Joëls M, Holsboer F. Stress and the brain: from adaptation to disease. Nat Rev Neurosci. 2005;6(6):463-475.

2. Holsboer F. The corticosteroid receptor hypothesis of depression. Neuropsychopharmacology. 2000;23(5):477-501.

3. Ulrich-Lai YM, Herman JP. Neural regulation of endocrine and autonomic stress responses. Nat Rev Neurosci. 2009;10(6):397409.

4. Stratmann M, Schibler U. Properties, entrainment, and physiological functions of mammalian peripheral oscillators. J Biol Rhythms. 2006;21(6):494-506.

5. Barclay JL, Tsang AH, Oster H. Interaction of central and peripheral clocks in physiological regulation. Prog Brain Res. 2012;199:163181.

6. Dickmeis T. Glucocorticoids and the circadian clock. J Endocrinol. 2009;200(1):3-22.

7. Kalsbeek A, van der Spek R, Lei J, Endert E, Buijs RM, Fliers E. Circadian rhythms in the hypothalamo-pituitary-adrenal (HPA) axis. Mol Cell Endocrinol. 2012;349(1):20-29.

8. Moore RY, Eichler VB. Loss of a circadian adrenal corticosterone rhythm following suprachiasmatic lesions in the rat. Brain Res. $1972 ; 42(1): 201-206$.

9. Oster H, Damerow S, Hut RA, Eichele G. Transcriptional profiling in the adrenal gland reveals circadian regulation of hormone biosynthesis genes and nucleosome assembly genes. J Biol Rhythms. 2006;21(5):350-361.

10. Oster H, Damerow S, Kiessling S, et al. The circadian rhythm of glucocorticoids is regulated by a gating mechanism residing in the adrenal cortical clock. Cell Metab. 2006;4(2):163-173.

11. Son GH, Chung S, Choe HK, et al. Adrenal peripheral clock controls the autonomous circadian rhythm of glucocorticoid by causing rhythmic steroid production. Proc Natl Acad Sci USA. 2008; 105(52):20970-20975.

12. Buhr ED, Takahashi JS. Molecular components of the Mammalian circadian clock. Handb Exp Pharmacol. 2013;217:3-27.

13. Oster H. The genetic basis of circadian behavior. Genes Brain Behav. 2006;5 Suppl 2:73-79.

14. Bunger MK, Wilsbacher LD, Moran SM, et al. Mop3 is an essential component of the master circadian pacemaker in mammals. Cell. 2000;103(7):1009-1017.

15. Ko CH, Yamada YR, Welsh DK, et al. Emergence of noise-induced oscillations in the central circadian pacemaker. PLoS Biol. 2010; 8(10):e1000513.

16. Balsalobre A, Brown SA, Marcacci L, et al. Resetting of circadian time in peripheral tissues by glucocorticoid signaling. Science. 2000 ; 289(5488):2344-2347.

17. Segall LA, Perrin JS, Walker CD, Stewart J, Amir S. Glucocorticoid rhythms control the rhythm of expression of the clock protein, Period2, in oval nucleus of the bed nucleus of the stria terminalis and central nucleus of the amygdala in rats. Neuroscience. 2006;140(3): $753-757$.

18. So AY, Bernal TU, Pillsbury ML, Yamamoto KR, Feldman BJ. Glu- 
cocorticoid regulation of the circadian clock modulates glucose homeostasis. Proc Natl Acad Sci USA. 2009;106(41):17582-17587.

19. Cheon S, Park N, Cho S, Kim K. Glucocorticoid-mediated Period2 induction delays the phase of circadian rhythm. Nucleic Acids Res. 2013;41(12):6161-6174.

20. Lamia KA, Papp SJ, Yu RT, et al. Cryptochromes mediate rhythmic repression of the glucocorticoid receptor. Nature. 2011;480(7378): 552-556.

21. Peckett AJ, Wright DC, Riddell MC. The effects of glucocorticoids on adipose tissue lipid metabolism. Metabolism. 2011;60(11): 1500-1510.

22. McEwen BS, Sapolsky RM. Stress and cognitive function. Curr Opin Neurobiol. 1995;5(2):205-216.

23. Parylak SL, Koob GF, Zorrilla EP. The dark side of food addiction. Physiol Behav. 2011;104(1):149-156.

24. Castagne V, Moser P, Roux S, Porsolt RD. Rodent models of depression: forced swim and tail suspension behavioral despair tests in rats and mice. Curr Protoc Pharmacol. 2010; Chapter 5:Unit 58.

25. Hänninen L, Pastell M. CowLog: open-source software for coding behaviors from digital video. Behav Res Methods. 2009;41(2):472476

26. Swiergiel AH, Leskov IL, Dunn AJ. Effects of chronic and acute stressors and CRF on depression-like behavior in mice. Behav Brain Res. 2008;186(1):32-40.

27. LeGates TA, Altimus CM, Wang H, et al. Aberrant light directly impairs mood and learning through melanopsin-expressing neurons. Nature. 2012;491(7425):594-598.

28. Barclay JL, Shostak A, Leliavski A, et al. High fat diet-induced hyperinsulinemia and tissue-specific insulin resistance in Cry deficient mice. Am J Physiol Endocrinol Metab. 2013;304(10):E1053E1063.

29. Bartolomucci A, Pederzani T, Sacerdote P, Panerai AE, Parmigiani S, Palanza P. Behavioral and physiological characterization of male mice under chronic psychosocial stress. Psychoneuroendocrinology. 2004;29(7):899-910.

30. Abraham D, Dallmann R, Steinlechner S, Albrecht U, Eichele G, Oster H. Restoration of circadian rhythmicity in circadian clockdeficient mice in constant light. J Biol Rhythms. 2006;21(3):169_ 176.

31. Cavigelli SA, Monfort SL, Whitney TK, Mechref YS, Novotny M, McClintock MK. Frequent serial fecal corticoid measures from rats reflect circadian and ovarian corticosterone rhythms. J Endocrinol. 2005; 184(1):153-163.

32. Karpac J, Ostwald D, Bui S, Hunnewell P, Shankar M, Hochgeschwender U. Development, maintenance, and function of the adrenal gland in early postnatal proopiomelanocortin-null mutant mice. En docrinology. 2005;146(6):2555-2562.

33. Ishida A, Mutoh T, Ueyama T, et al. Light activates the adrenal gland: timing of gene expression and glucocorticoid release. Cell Metab. 2005;2(5):297-307.

34. Andrews JL, Zhang X, McCarthy JJ, et al. CLOCK and BMAL1 regulate $\mathrm{MyoD}$ and are necessary for maintenance of skeletal muscle phenotype and function. Proc Natl Acad Sci USA. 2010;107(44): 19090-19095.

35. Bunger MK, Walisser JA, Sullivan R, et al. Progressive arthropathy in mice with a targeted disruption of the Mop3/Bmal-1 locus. Genesis. 2005;41(3):122-132.

36. Arai I, Tsuyuki Y, Shiomoto H, Satoh M, Otomo S. Decreased body temperature dependent appearance of behavioral despair in the forced swimming test in mice. Pharmacol Res. 2000;42(2):171-176.

37. Park SY, Walker JJ, Johnson NW, Zhao Z, Lightman SL, Spiga F. Constant light disrupts the circadian rhythm of steroidogenic proteins in the rat adrenal gland. Mol Cell Endocrinol. 2013;371(1 2):114-123.

38. Chiba S, Numakawa T, Ninomiya M, Richards MC, Wakabayashi C, Kunugi H. Chronic restraint stress causes anxiety- and depression-like behaviors, downregulates glucocorticoid receptor expres- sion, and attenuates glutamate release induced by brain-derived neurotrophic factor in the prefrontal cortex. Prog Neuropsychopharmacol Biol Psychiatry. 2012;39(1):112-119.

39. Strekalova T, Couch Y, Kholod N, et al. Update in the methodology of the chronic stress paradigm: internal control matters. Behav Brain Funct. 2011;7:9.

40. Yi LT, Li J, Li HC, et al. Antidepressant-like behavioral, neurochemical and neuroendocrine effects of naringenin in the mouse repeated tail suspension test. Prog Neuropsychopharmacol Biol Psychiatry. 2012;39(1):175-181.

41. Rudic RD, McNamara P, Curtis AM, et al. BMAL1 and CLOCK, two essential components of the circadian clock, are involved in glucose homeostasis. PLoS Biol. 2004;2(11):e377.

42. Turek FW, Joshu C, Kohsaka A, et al. Obesity and metabolic syndrome in circadian Clock mutant mice. Science. 2005;308(5724): 1043-1045.

43. Doi M, Takahashi Y, Komatsu R, et al. Salt-sensitive hypertension in circadian clock-deficient Cry-null mice involves dysregulated adrenal Hsd3b6. Nat Med. 2010;16(1):67-74.

44. Yang S, Liu A, Weidenhammer A, et al. The role of $\mathrm{mPer} 2$ clock gene in glucocorticoid and feeding rhythms. Endocrinology. 2009; 150(5):2153-2160.

45. Shearman LP, Sriram S, Weaver DR, et al. Interacting molecular loops in the mammalian circadian clock. Science. $2000 ; 288(5468)$ : 1013-1019.

46. Bornstein SR. Predisposing factors for adrenal insufficiency. N Engl J Med. 2009;360(22):2328-2339.

47. Shimba S, Ishii N, Ohta Y, et al. Brain and muscle Arnt-like protein-1 (BMAL1), a component of the molecular clock, regulates adipogenesis. Proc Natl Acad Sci USA. 2005;102(34):12071-12076.

48. Torres-Farfan C, Abarzua-Catalan L, Valenzuela FJ, et al. Cryptochrome 2 expression level is critical for adrenocorticotropin stimulation of cortisol production in the capuchin monkey adrenal. Endocrinology. 2009;150(6):2717-2722.

49. Cho H, Zhao X, Hatori M, et al. Regulation of circadian behaviour and metabolism by REV-ERB- $\alpha$ and REV-ERB- $\beta$. Nature. 2012; 485(7396):123-127.

50. Curtis AM, Cheng Y, Kapoor S, Reilly D, Price TS, Fitzgerald GA. Circadian variation of blood pressure and the vascular response to asynchronous stress. Proc Natl Acad Sci USA. 2007;104(9):3450_ 3455 .

51. Rogóz Z, Skuza G, Leskiewicz M, Budziszewska B. Effects of coadministration of fluoxetine or tianeptine with metyrapone on immobility time and plasma corticosterone concentration in rats subjected to the forced swim test. Pharmacol Rep. 2008;60(6):880 888 .

52. Mitchell JB, Meaney MJ. Effects of corticosterone on response consolidation and retrieval in the forced swim test. Behav Neurosci. 1991;105(6):798-803.

53. Tronche F, Kellendonk C, Kretz O, et al. Disruption of the glucocorticoid receptor gene in the nervous system results in reduced anxiety. Nat Genet. 1999;23(1):99-103.

54. Granados-Fuentes D, Tseng A, Herzog ED. A circadian clock in the olfactory bulb controls olfactory responsivity. J Neurosci. 2006, 26(47):12219-12225

55. Stewart LQ, Roper JA, Young WS, 3rd, O'Carroll AM, Lolait SJ. Pituitary-adrenal response to acute and repeated mild restraint, forced swim and change in environment stress in arginine vasopressin receptor 1b knockout mice. J Neuroendocrinol. 2008;20(5): 597-605.

56. Dubreucq S, Matias I, Cardinal P, et al. Genetic dissection of the role of cannabinoid type-1 receptors in the emotional consequences of repeated social stress in mice. Neuropsychopharmacology. 2012; 37(8):1885-1900.

57. Piazza PV, Barrot M, Rougé-Pont F, et al. Suppression of glucocorticoid secretion and antipsychotic drugs have similar effects on the 
mesolimbic dopaminergic transmission. Proc Natl Acad Sci USA. 1996;93(26): 15445-15450.

58. Rovbal K, Theobold D, Graham A, et al. Mania-like behavior induced by disruption of CLOCK. Proc Natl Acad Sci USA. 2007; 104(15):6406-6411.
59. Hampp G, Ripperger JA, Houben T, et al. Regulation of monoamine oxidase A by circadian-clock components implies clock influence on mood. Curr Biol. 2008;18(9):678-683.

60. Albrecht U. Circadian clocks and mood-related behaviors. Handb Exp Pharmacol. 2013;217:227-239. 
Leliavski et al. "Impaired stress response in Arntl-/- mice" - Supplemental data

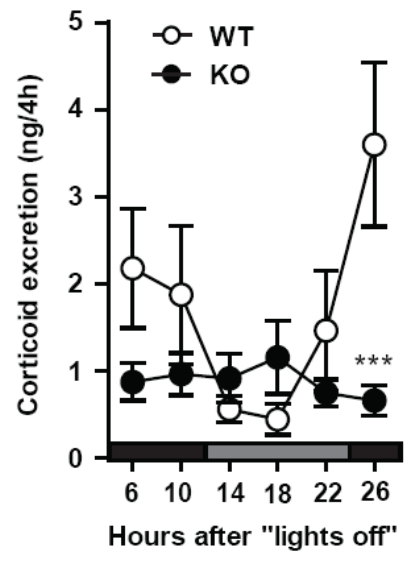

Supplemental Figure 1. 24-hour profile of corticoid excretion in fecal samples from WT and KO mice on the first day in DD $(n=5-6) .{ }^{* *} \mathrm{P}<.001$ (2-way ANOVA with Bonferroni post-hoc test).

A

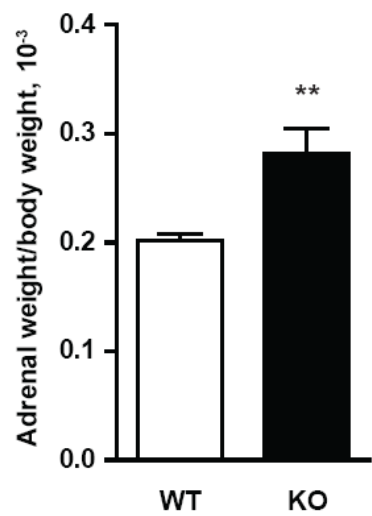

B

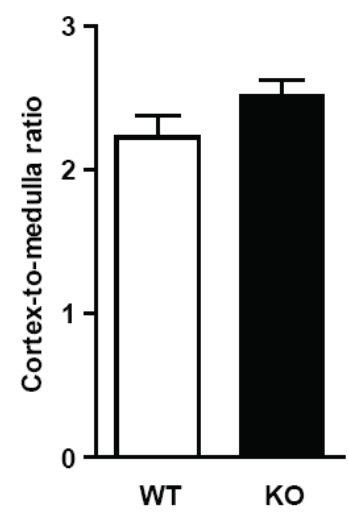

Supplemental Figure 2. Anatomical examination of Arntl-deficient adrenal glands. (A) Relative adrenal weight in WT and KO mice $(n=9-13)$. (B) Adrenal cortex-to-medulla ratio in KO mice and age-matched WT controls $(n=5-6)$. 
A

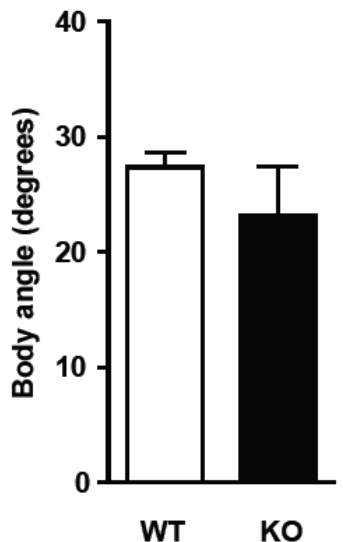

B

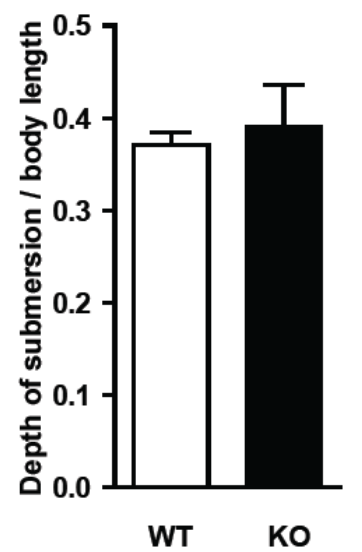

C

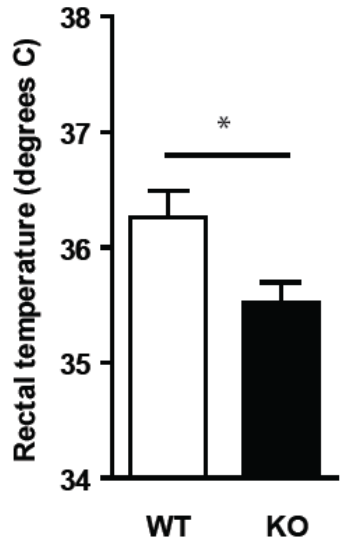

Supplemental Figure 3. Body axis angle $(A)$ and submersion in water $(B)$ of WT and KO mice during immobility periods in the FST. (C) Rectal temperature measured in WT and KO mice at ZT12. ${ }^{*} \mathrm{P}<.05$ (Student's t-test).

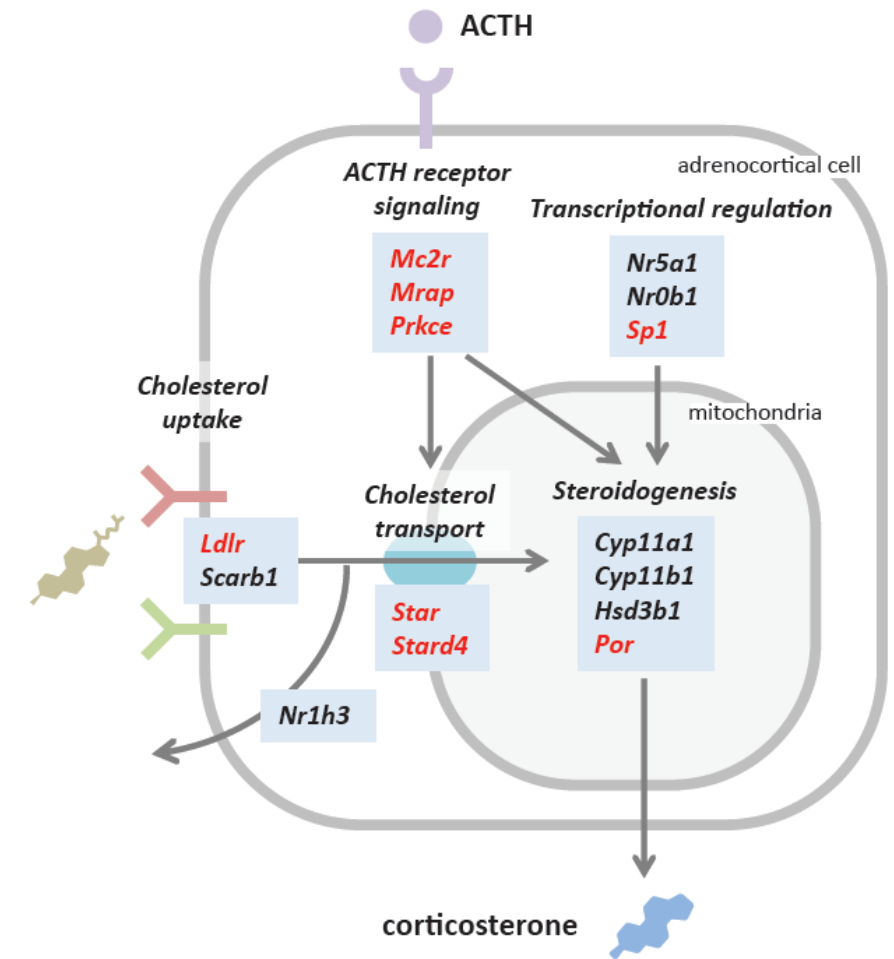

Supplemental Figure 4. Schematic representation of tested genes involved in regulation of adrenocortical functions. Rhythmically expressed genes are labeled in red. 

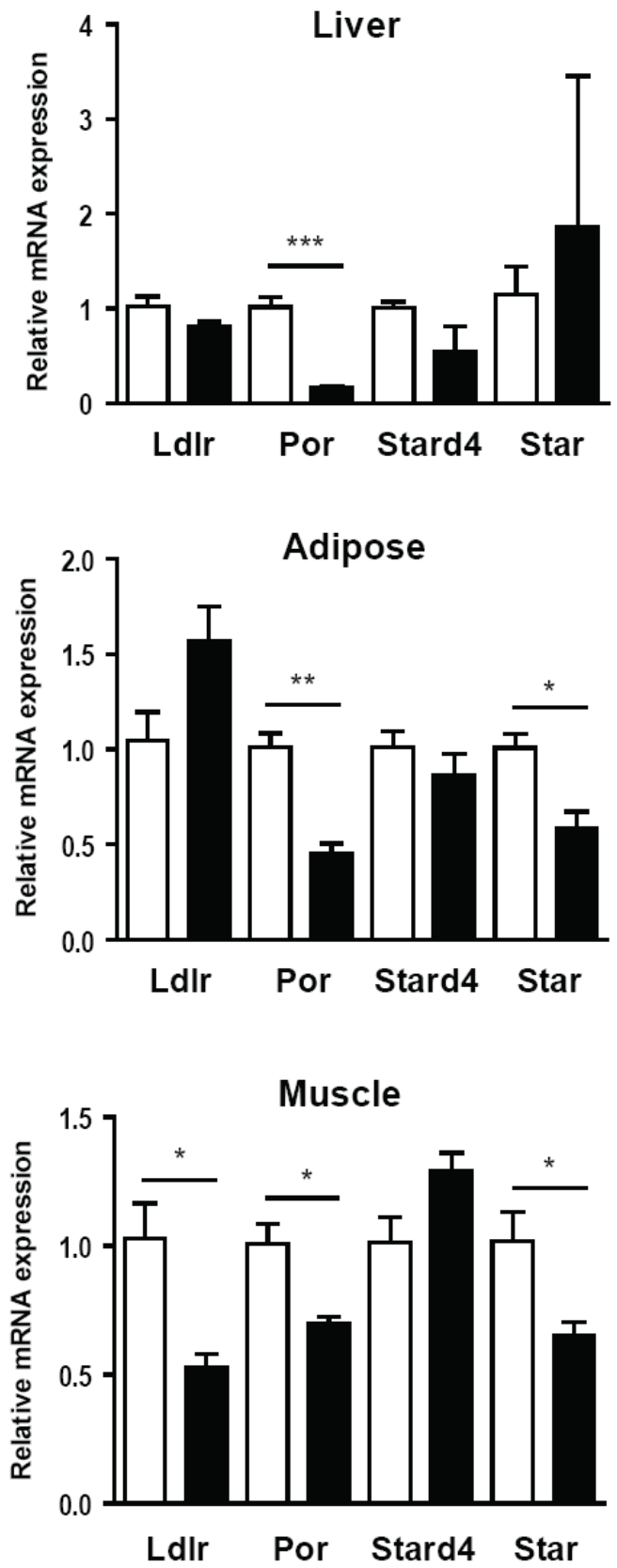

Supplemental Figure 5. mRNA levels of genes of interest in liver, epididymal adipose tissue and skeletal muscle from WT and KO mice at $48 \mathrm{~h}$ after "lights off" $(\mathrm{n}=3-4) .{ }^{*} \mathrm{P}<.05,{ }^{* *} \mathrm{P}<.01,{ }^{* *} \mathrm{P}<.001$ (Student's t-test). 
Supplemental Table 1. Primer sequences used for quantitative RT-PCR

\begin{tabular}{|c|c|c|}
\hline Gene & Forward primer (5' to 3') & Reverse primer (5' to 3') \\
\hline Star & TTGGGCATACTCAACAACCA & GAAACACCTTGCCCACATCT \\
\hline$M c 2 r$ & AAGCCTCGTGGCAGTTTTGAA & AGGATGAACATGCAGTCAATGAT \\
\hline$L d l r$ & TGACTCAGACGAACAAGGCTG & ATCTAGGCAATCTCGGTCTCC \\
\hline Prkce & GGGGTGTCATAGGAAAACAGG & GACGCTGAACCGTTGGGAG \\
\hline Por & ATGGGGGACTCTCACGAAGAC & TCTTGCTGAACTCCGGTATCTC \\
\hline Stard4 & TGTTTGGTATGGAGAGTGTGGA & GTCACAGCAGAGACTGACATTG \\
\hline Scarb1 & TTTGGAGTGGTAGTAAAAAGGGC & TGACATCAGGGACTCAGAGTAG \\
\hline Nr5al & GTAACTGAACTGGTCAAACCCC & AAGCCTGGCTAGTGCATGTC \\
\hline $\mathrm{NrOb1}$ & CGGATGATGCAGAGAGAGTACC & AATGATGGGCCTGAAAAAGAGTT \\
\hline Spl & GCCGCCTTTTCTCAGACTC & TTGGGTGACTCAATTCTGCTG \\
\hline$N r 1 h 3$ & CTCAATGCCTGATGTTTCTCCT & TCCAACCCTATCCCTAAAGCAA \\
\hline Cyplla1 & AGGTCCTTCAATGAGATCCCTT & TCCCTGTAAATGGGGCCATAC \\
\hline Cyp11b1 & CTGAACCCAAATGTTCTGTCACC & CAAAGTCCCTTGCTATCCCATC \\
\hline$H s d 3 b 1$ & AGCATCCAGACACTCTCATC & GGAGCTGGTATGATATAGGGTA \\
\hline Mrap & ACTGTCATGGCCAACGG & AGTGTGAGGCCAGCTGAT \\
\hline Eeflal & TGCCCCAGGACACAGAGACTTCA & AATTCACCAACACCAGCAGCAA \\
\hline
\end{tabular}




\subsection{Generation and evaluation of adrenocortical clock-deficient (ACD) mice}

\subsubsection{Generation of conditional knockout mice deficient in adrenocortical circadian clock}

To specifically disrupt the molecular clock in the adrenal cortex, I used the Cre-loxP gene targeting system that allows deleting target region of a gene of interest flanked with loxP sites ("floxed") by the Cre recombinase expressed under control of a tissue-specific promoter.

Among the core circadian clock proteins, BMAL1 is the only non-redundant and essential component as has been strongly supported by the fact that $\mathrm{Bmall}^{-{ }_{-}}$mice are completely arrhythmic at molecular and behavioral levels (Bunger et al., 2000; Ko et al., 2010). Therefore I selected BMAL1 as a suitable molecular target for deleting clock function in the adrenal cortex. For this purpose I used Bmal $1^{f l f l}$ mice that were generated by C. Weitz and his colleagues (Storch et al., 2007). In this mouse line, loxP sites flank the exon encoding the BMAL1 basic helix-loop-helix (bHLH) domain, which is essential for heterodimerization with CLOCK or NPAS2 to induce transcription (Huang et al., 2012). Upon the action of Cre recombinase, deletion of the bHLH-encoding DNA fragment results in a mutation nearly identical to the original null mutation in Bmall knockout mice (Bunger et al., 2000; Storch et al., 2007).

To target the Bmall gene in adrenocortical cells I obtained a commercially available Cypllal-CreGFP knock-in mouse line, in which CRE recombinase targets steroidogenic cells (adrenocortical cells and testicular Leydig cells) (www.gudmap.org; O'Hara et al. 2013, in press). The Cypllal gene encodes the cholesterol side-chain cleavage enzyme $(\mathrm{P} 450 \mathrm{scc})$ that initiates the process of steroidogenesis by converting cholesterol to pregnenolone, which makes it a good candidate for gene
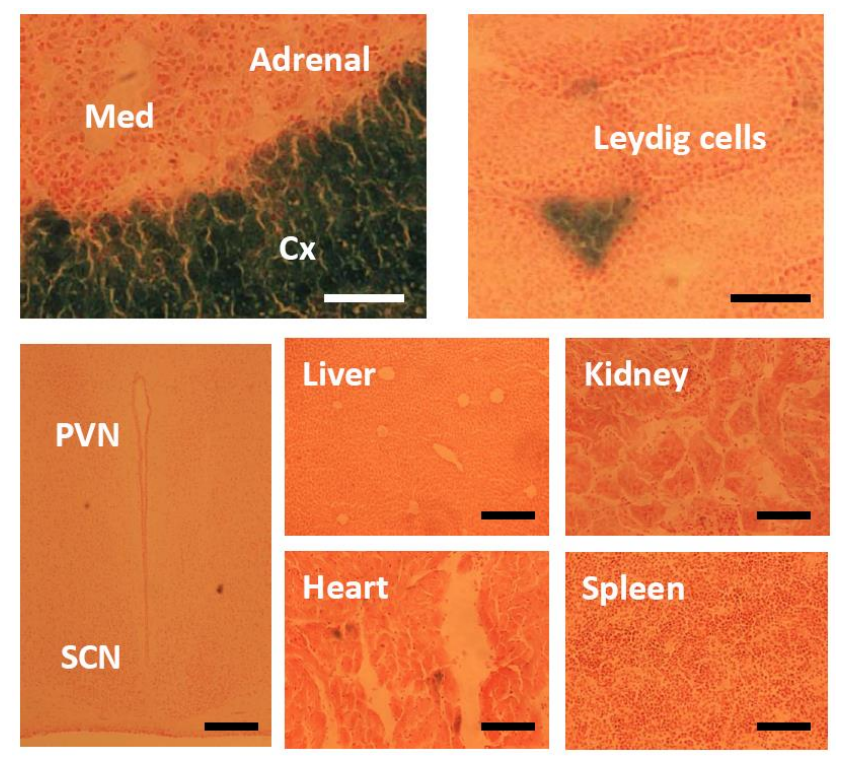

Figure 8. $\beta$-galactosidase staining of tissues from Cyp 1 la1 ${ }^{\text {Cre/t}} ;$ ROSA26R $^{\text {lacZ/+ }}$ male mice. Scale bar: $100 \mu \mathrm{m}$ (upper two images), $200 \mu \mathrm{m}$ (lower part). $\mathrm{Cx}-$ cortex, Med - medulla. targeting in steroid-producing tissues. Indeed, after crossing these mice with ROSA26RlacZ reporter mice I observed intense $\beta$-galactosidase staining in the adrenal cortex and testosterone-producing Leydig cells in testes - two main sites of 
Cypllal expression (Figure 8, upper part). Very sporadic CRE activity was detected in single cerebellar granule cells and few cardiomyocytes, whereas other analyzed tissues, including the SCN, remained $\beta$-galactosidase-negative (Figure 8 , lower part). Thus I concluded that this Cre driver line is suitable for generation of adrenocortical clock-deficient ( $A C D$, or mutant) mice by crossing the mice with Bmall flfl animals. Nevertheless, a potential clock disruption in Leydig cells of these mice should be taken into account. In further experiments, Cypllal ${ }^{\mathrm{Cre} /+}$ male littermates were used as controls, since the targeting vector containing the GFP-Cre cassette disrupts the exon containing the ATG translation start site of the Cypllal gene. Although it was reported that the heterozygous animals are viable, fertile, normal in size and do not display any gross physical or behavioral abnormalities (http://jaxmice.jax.org/strain/010988.html), they may develop a mild phenotype in steroid biosynthesis (Hu et al., 2002). Indeed, the amplitude of plasma CORT rhythms in control Cypl lal ${ }^{\mathrm{Cr} / \mathrm{t}}$ animals was moderately reduced compared to wild-type mice (Figure 9).

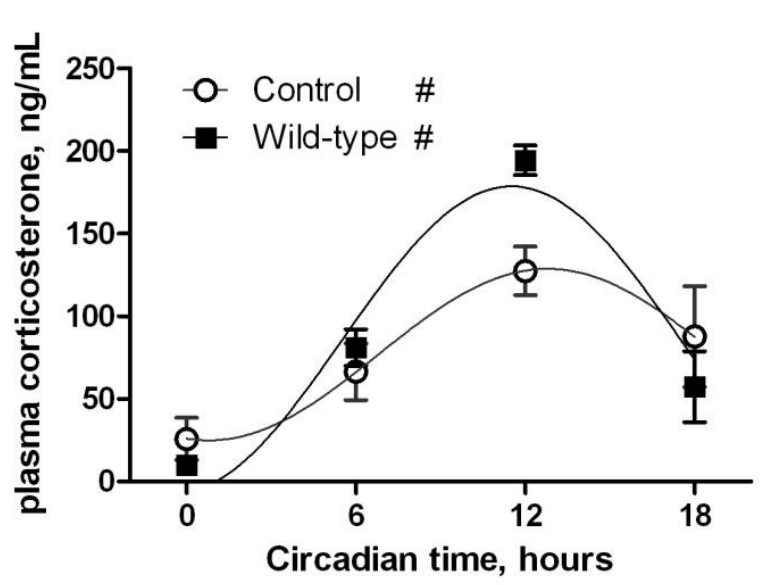

Figure 9. Plasma CORT production in wild-type and Cypllal ${ }^{\text {Crelt }}$ control male mice ( $\mathrm{n}=3$ per group). 2-way ANOVA revealed significant effect of time $(\mathrm{p}<0.0001)$. \#: $\mathrm{p}<0.05$, CircWave analysis; controls $(\mathrm{p}=0.012)$, wild-types $(\mathrm{p}<0.001)$.
To verify the efficiency of Bmall deletion in adrenocortical cells, I analyzed the expression of Bmall mRNA and protein in the adrenal cortex of control and mutant mice using $\quad$ qRT-PCR and immunohistochemistry methods, respectively. To estimate the efficiency of Bmall deletion by qRT-PCR I used primers targeting the floxed DNA sequence of the Bmall gene (Husse et al., 2011). Bmall mRNA transcription in the adrenal cortex at the peak of its expression, circadian time (CT) 0, was significantly reduced to $15.6 \pm 0.7 \%$ of that in control adrenals (Figure 10A). Similarly, immunohistochemical staining of BMAL1 protein in adrenals revealed a marked reduction of BMAL1-positive cells in the adrenal cortex of mutant mice $(13.5 \pm 5.2 \%$ of controls; Figure 10B), whereas the expression of BMAL1 protein in adrenal medullar cells was not affected.

To ensure that deletion of BMAL1 leads to disruption of the circadian clock activity in adrenocortical cells of the mutants, I measured circadian rhythms in expression of clock genes, such as Bmall, Dbp and Nrldl (which encodes REV-ERB $\alpha$ ), in control and mutant animals on the second day in DD. As expected, Bmall transcript was rhythmic in the kidney of the mutant mice, whereas the rhythmicity of a (non-functional) Bmall mRNA was drastically dampened in the adrenal cortex of the mutants 
(Figure 10C). Similarly, both $\mathrm{Dbp}$ and Nrldl transcripts were down-regulated and arrhythmic in the adrenal cortex of the mutant mice (Figure 10C), whereas no significant changes between controls and mutants were observed in the expression of the clock genes in the kidney (Figure 10D).

A

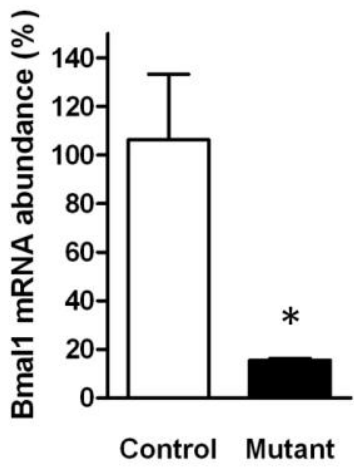

B BMAL1-IR

\section{Control Mutant}

$\mathrm{Cx}$
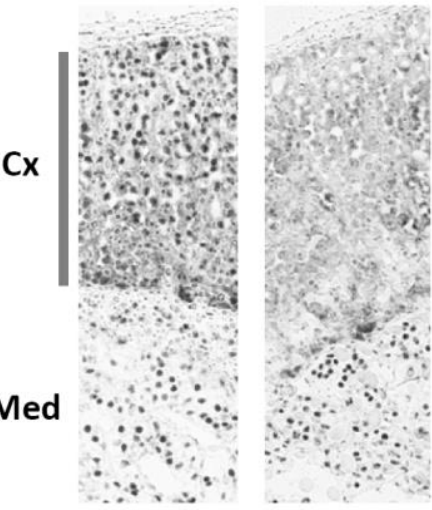

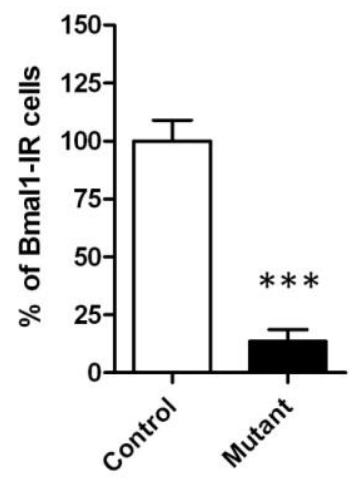

\section{Adrenal cortex}
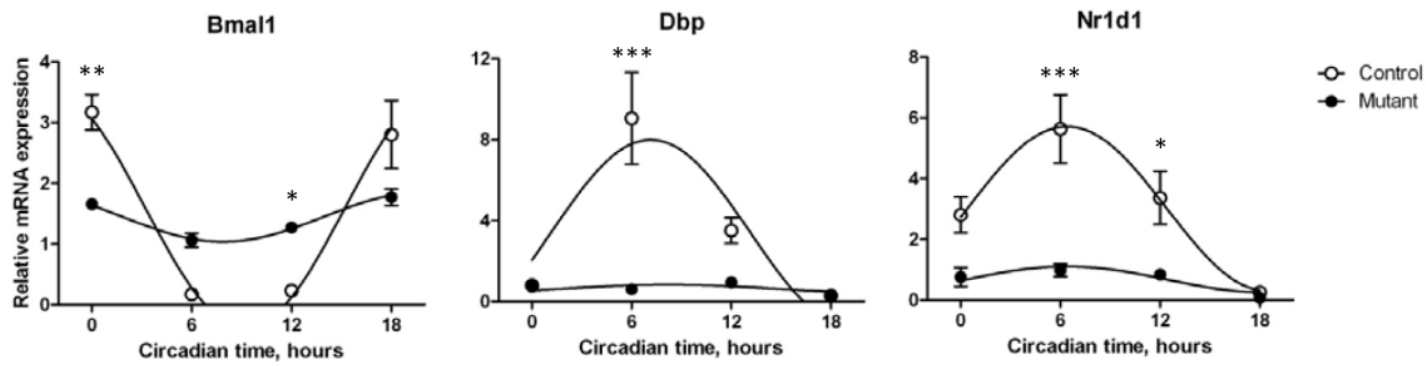

D Kidney
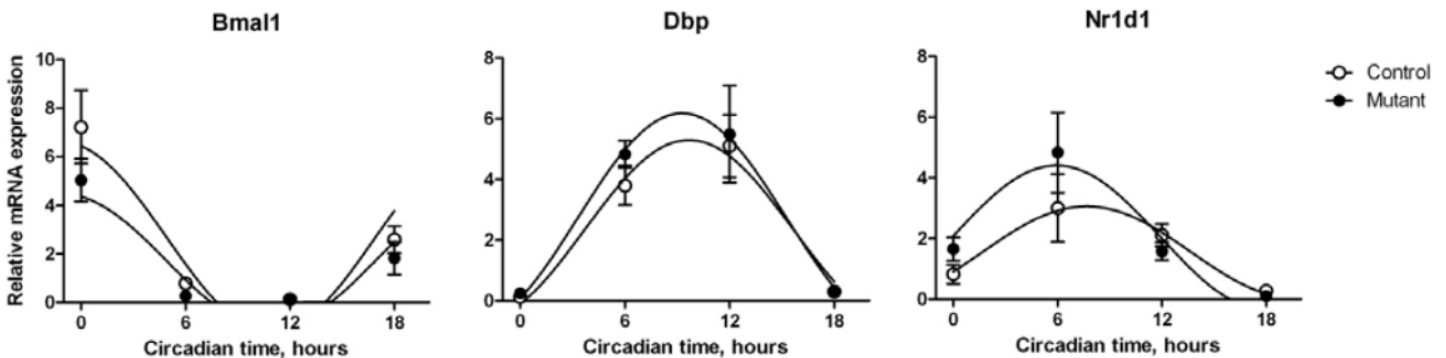

Figure 10. Quantification of the conditional knockout efficiency. (A) Bmall mRNA expression in the adrenal cortex of control and mutant mice $(\mathrm{n}=3)$. *: $\mathrm{p}=0.03$. (B) Immunohistochemical staining of BMAL1 protein in adrenals of control and mutant mice ( $\mathrm{n}=6)$. ***: $\mathrm{p}<0.001$. IR—immunoreactivity. ( $\mathrm{C}$ and $\mathrm{D}$ ) Expression of clock and clock-controlled genes in the adrenal cortex and kidney of control and mutant mice ( $\mathrm{n}=3$ per group). *: $\mathrm{p}<0.05, * *: \mathrm{p}<0.01,{ }^{* * *}: \mathrm{p}<0.001$ (2-way ANOVA with Bonferroni post-hoc test). Circwave analysis revealed significant rhythms in all cases ( $\mathrm{p}<0.001)$, except of $D b p$ and $N r l d l$ in adrenal cortex of mutants. 


\subsubsection{Circadian hormone production in the adrenocortical clock-deficient mice}

To further understand how a compromised circadian clock in the adrenal cortex affects adrenal endocrine functions, I measured circadian rhythms of adrenocortical hormones - corticosterone (CORT) and aldosterone - in plasma using radioimmune assays. Additionally, CORT immunoreactivity was measured in fecal extracts of control and mutant mice under light-dark (LD) conditions and in constant darkness (DD). Interestingly, daily rhythms of fecal corticoid excretion were not altered in the mutant mice both in LD and DD conditions, compared to control animals (Figure 11A). In accordance, circadian variation of plasma CORT was preserved in ACD mice (Figure $11 B)$. Even a mild elevation of plasma and fecal CORT levels was observed around the peak of CORT release in the beginning of the activity phase in mutant animals (Figure 11, A and B).
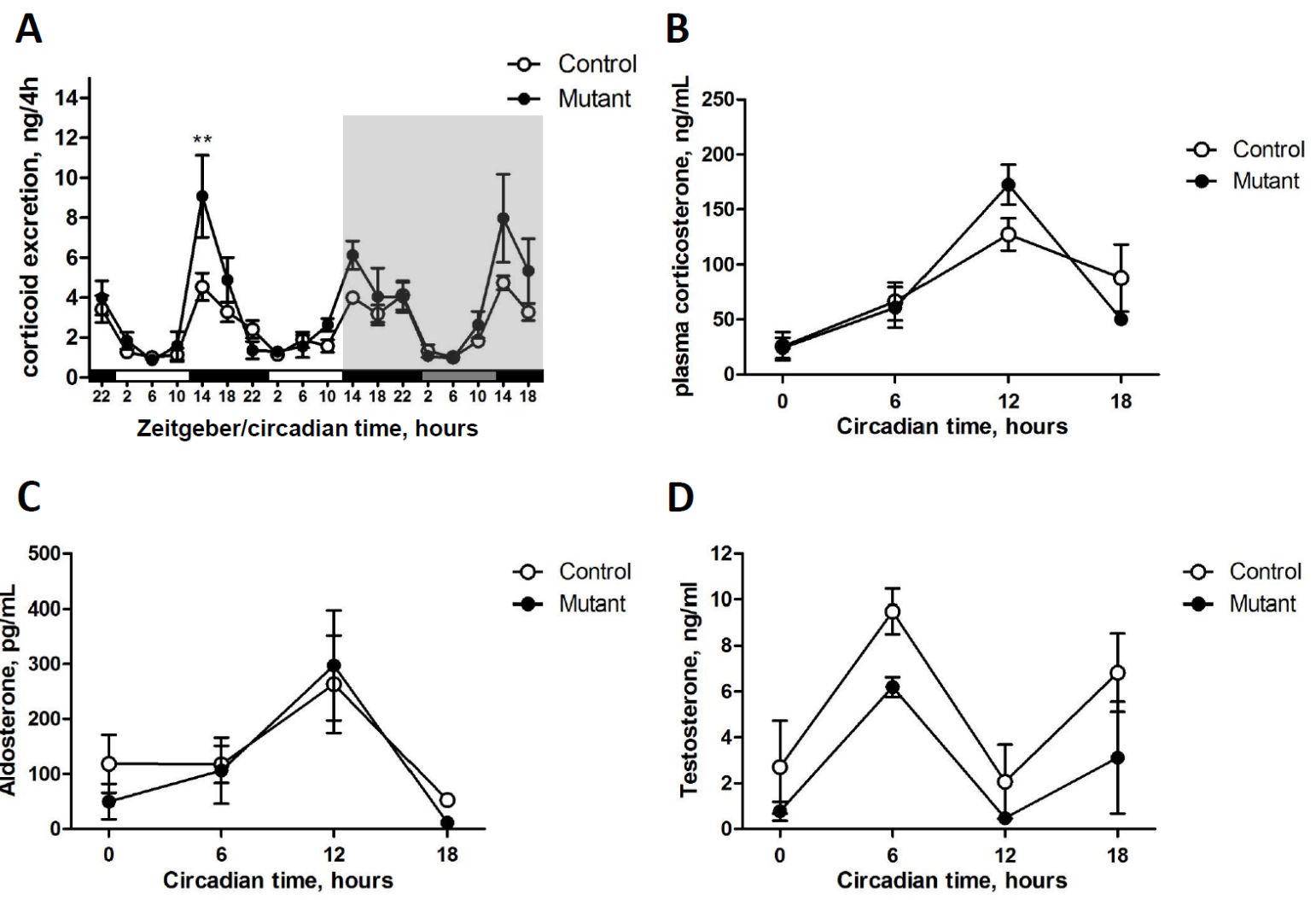

Figure 11. Steroid hormone production in control and ACD mice. (A) CORT immunoreactivity in fecal extracts $(n=4)$. Shaded area indicates the time when mice were kept in constant darkness. (B-D) Circadian analysis of CORT (B), aldosterone (C) and testosterone (D) levels in plasma of control and mutant mice $(n=3)$. In panel A, repeated measures 2-way ANOVA revealed significant effects of time $(\mathrm{p}<0.0001)$ and interaction between two factors (genotype and time) $(\mathrm{p}=0.027)$. Bonferroni posthoc test revealed increased CORT excretion in mutants at ZT14 ( $<<0.01$ ). Separate statistical analysis (repeated measures 2-way ANOVA) of fecal corticoid excretion during LD and DD conditions showed, in LD, there is significant effect of time $(\mathrm{p}<0.0001)$ and significant interaction between both factors $(p=0.004)$. In $\mathrm{DD}$, the effect of time is only significant $(\mathrm{p}<0.0001)$. In panel $\mathrm{B}$, 
significant effect of time ( $\mathrm{p}<0.001)$. In panel $\mathrm{C}$, 2-way ANOVA revealed significant effect of time $(\mathrm{p}=0.003)$. In panel D, 2-way ANOVA revealed significant effects of genotype $(\mathrm{p}=0.021)$ and time $(\mathrm{p}=0.001)$.

The CYP11A1 enzyme is required for synthesis of both CORT and aldosterone by adrenocortical cells of the zona fasciculata and zona glomerulosa, respectively. Moreover, circadian rhythms in aldosterone release have been reported (Doi et al., 2010). This implies that the circadian clock in aldosterone-producing cells is likely to be affected in the mutant mice. However, circadian rhythms of plasma aldosterone, analogous to CORT rhythms, are intact in the mutant mice (Figure 11C).

In addition I analyzed whether adrenocortical clock deficiency leads to altered circadian rhythms in expression of some adrenal transcripts involved in steroidogenesis that were previously found to be affected in Bmall knockout adrenals (section 2.1). The expression of Star mRNA was moderately down-regulated, whereas the changes in Ldlr and Por expression were not significant (Figure 12, upper row).
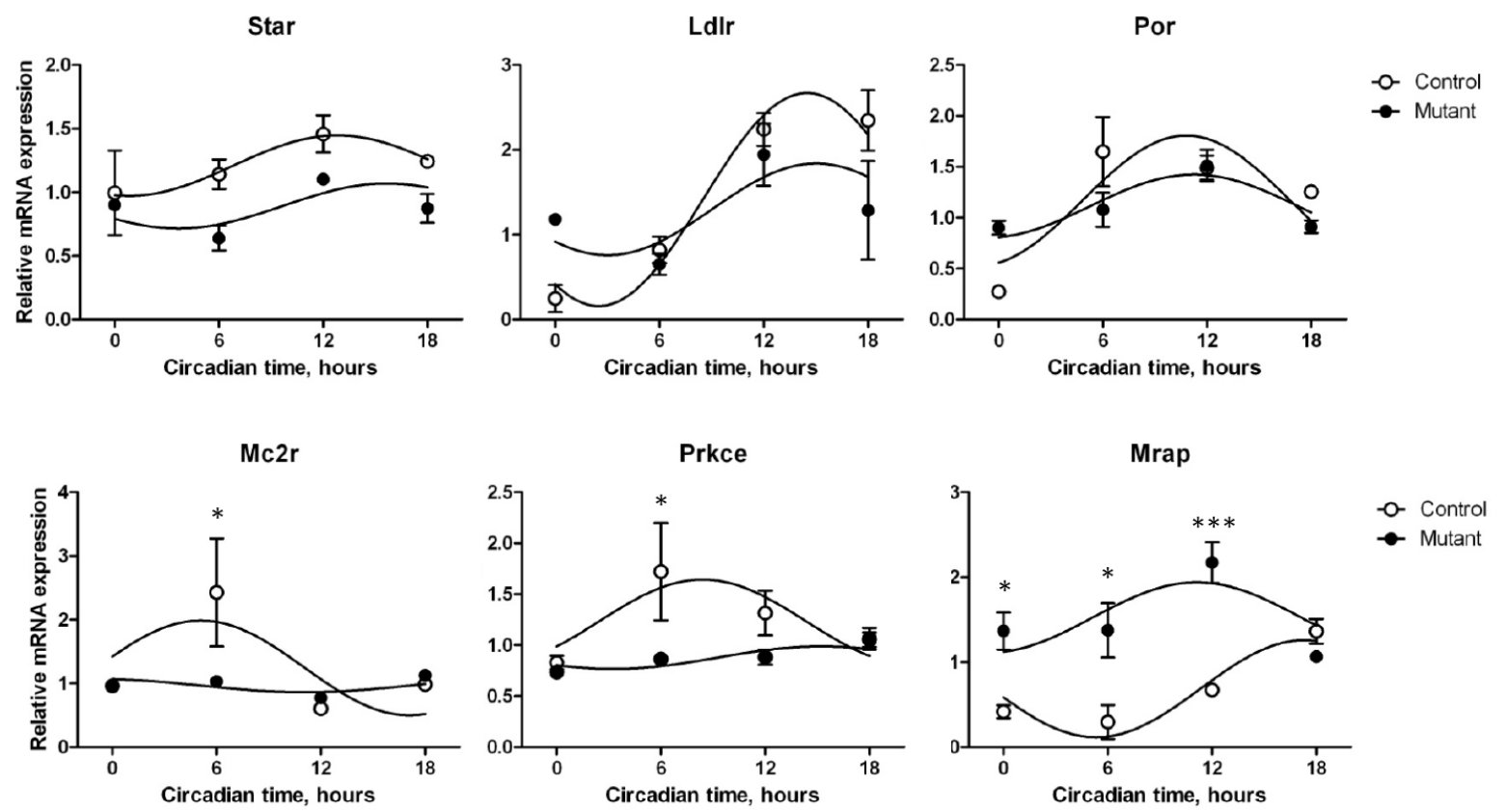

Figure 12. Expression of genes involved in steroidogenesis (upper row) and ACTH receptor signaling (lower row) in the adrenal cortex of control and ACD mice $(\mathrm{n}=3) . *$ : $<<0.05, * *: \mathrm{p}<0.01, * * *: \mathrm{p}<0.001$ (2-way ANOVA with Bonferroni post-hoc test). 2-way ANOVA revealed significant effect of time for $L d l r, P o r, M c 2 r$ and Mrap; significant effect of genotype for Star, Mrap and Prkce; significant interaction between two factors for Ldlr, Por and Mrap. 
One possible explanation for the fact that the adrenal CORT content in the mutant mice was not changed is that ACTH release from the pituitary and/or adrenal ACTH sensitivity are amplified in the mutant mice. Indeed, in $\mathrm{Bmall}^{-/-}$mice I observed that reduced responsiveness to ACTH coincided with up-regulated expression of the ACTH receptor (section 2.1). However, plasma ACTH measurements did not reveal any changes in circadian ACTH production in constant darkness in ACD mice, compared to the controls (Figure 13). I further analyzed the adrenal expression of genes that encode key components of the ACTH receptor signaling such as the ACTH receptor, MC2R, its accessory molecule MRAP and a subunit of the downstream protein kinase $\mathrm{C}$ epsilon (encoded by Prkce gene) (Figure 12, lower row). Importantly, all three transcripts have been previously shown to be expressed in a rhythmic fashion (Oster et al., 2006b; Park et al., 2013). Indeed, I confirmed that $M c 2 r$ and Mrap mRNAs are expressed in antiphase in the control mice. ACD mice showed a loss of circadian rhythmicity in the transcription of $M c 2 r$ and Prkce genes. Surprisingly, Mrap mRNA was highly up-

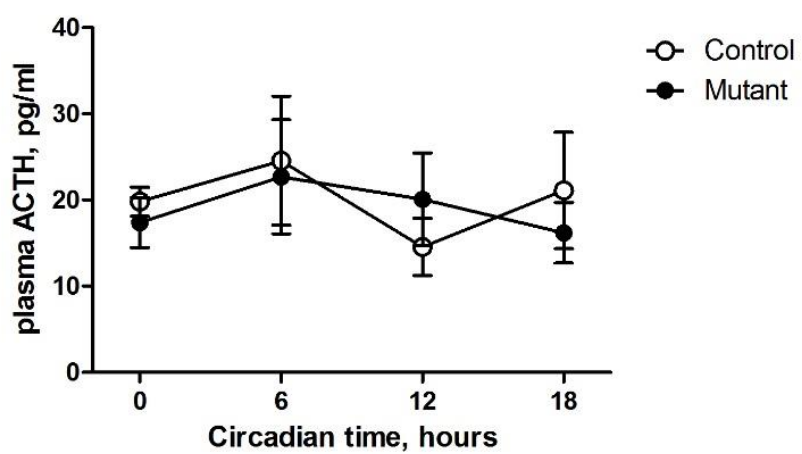

Figure 13. Plasma ACTH levels in control and ACD mice $(n=3-5)$.

regulated during the day and early night in circadian clock-deficient adrenals.

Finally, the tissue pattern of Cre expression in the mutant mice suggested that Bmall deficiency in Leydig cells in testes may alter testosterone production. 2-way ANOVA revealed modest reduction of plasma testosterone in mutant mice under DD conditions, compared to controls (Figure 11D).

Taken together, these results suggest that under basal conditions adrenocortical clock deficiency does not lead to significant abnormalities in corticosteroid production, though the expression of some genes involved in steroidogenesis and ACTH sensitivity is altered.

\subsubsection{Circadian behavioral resynchronization in response to a jet lag in the adrenocortical clock-deficient mice}

At the beginning of my thesis I had hypothesized that the adrenal clock may be important for stabilizing circadian phase against external perturbation, e.g. during stress response or resynchronization of the circadian rhythms after a phase shift of light-dark cycle (jet lag). As was shown above, global Bmall deficiency indeed results in compromised hormonal and behavioral responses to acute stress (section 2.1). Furthermore, previous studies demonstrated that the behavioral re-entrainment during experimental jet lag is altered in adrenalectomized mice as well as in wild-type mice with transplanted Per2/Cryl-deficient adrenals (Kiessling et al., 2010; Sage et al., 2004). 
I measured locomotor activity of control and mutant mice under LD conditions as well as in constant darkness. Analysis of wheel-running behavior revealed a moderate, but significant shortening of the endogenous period length in mutant mice in DD, but no changes in locomotor activity in LD (Figure 14). Further, ACD mice showed faster behavioral adaptation in a 6-hour jet lag paradigm (Figure 15).

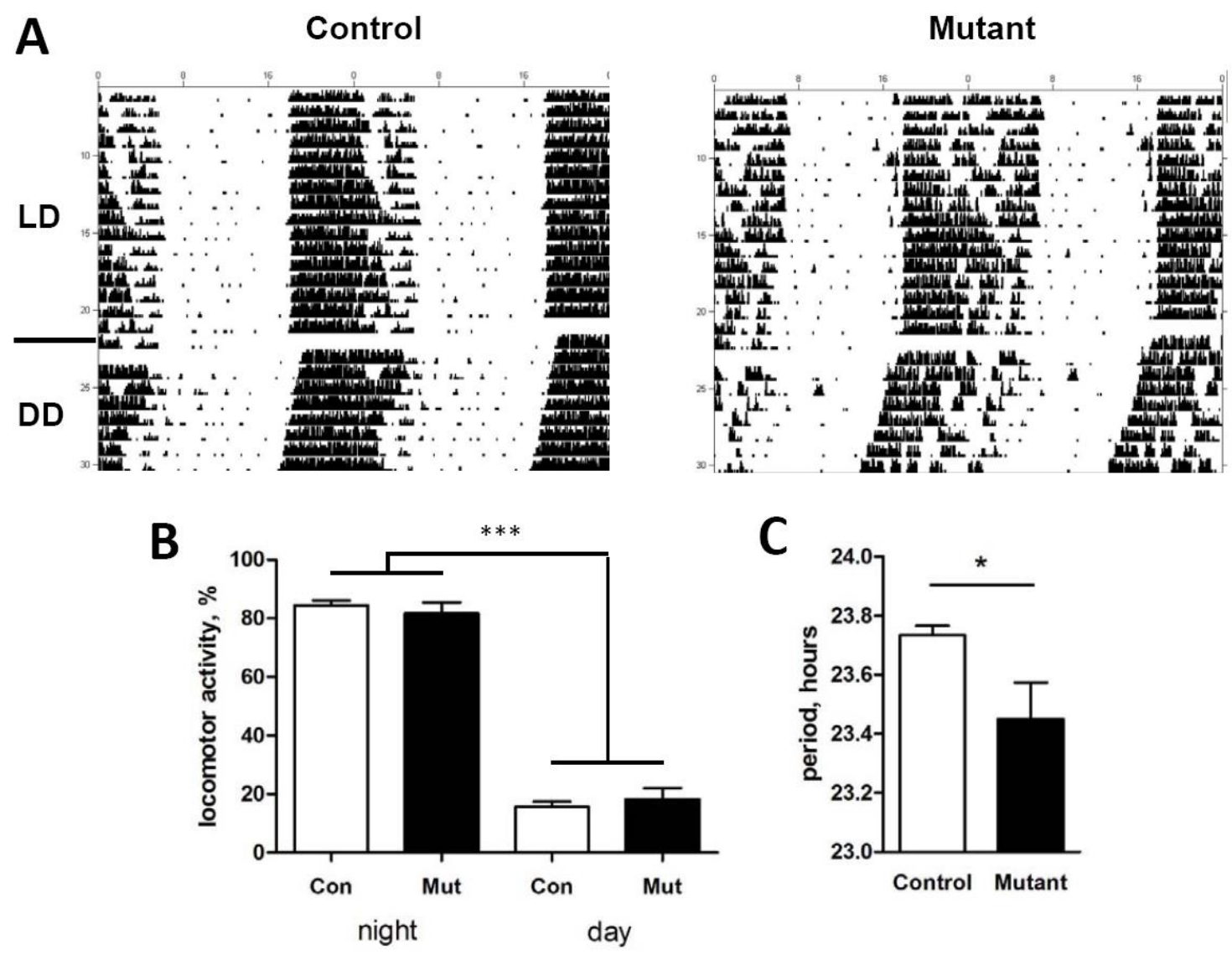

Figure 14. Locomotor behavior in control and mutant mice ( $n=6$ ). (A) Representative double-plotted actograms of control and ACD mice. Vertical bars represent wheel-running activity. Unplanned interruption of the recording is seen on day 23. (B) Locomotor activity during light and dark periods of LD cycle. (C) Endogenous period of locomotor activity in constant dark. *: p<0.05, ***: $<<0.001$ (unpaired t-test). 
Control
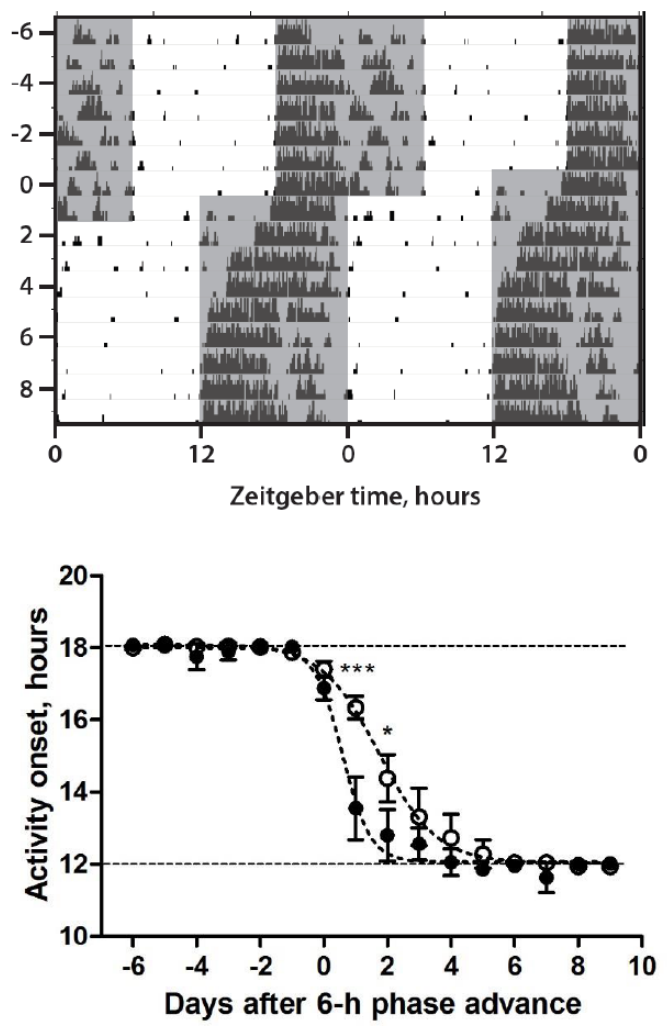

Mutant

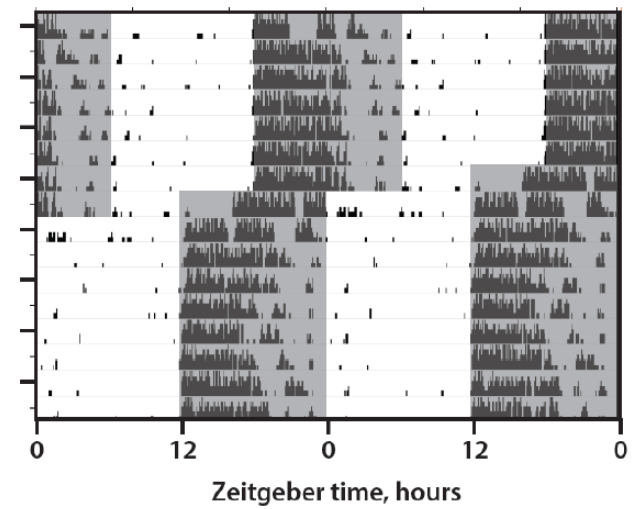

- - Control

-•- Mutant

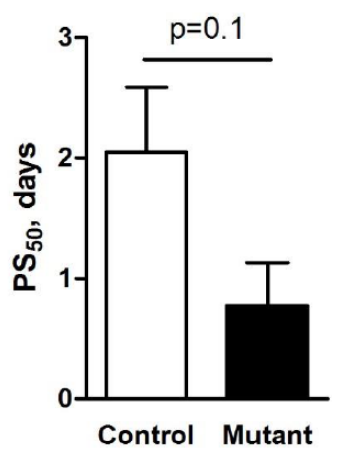

Figure 15. Behavioral re-entrainment in control and mutant mice following a 6-hr shift of the LD cycle (n=5). *: $\mathrm{p}<0.05$, ***: $\mathrm{p}<0.001$ (repeated measures 2-way ANOVA with Bonferroni post-hoc test). Average onsets of locomotor activity and $50 \%$ phase shifts $\left(\mathrm{PS}_{50}\right)$ in control and mutant mice during the jet lag. The $\mathrm{PS}_{50}$ value is defined a time at which half of the phase shift was completed. 


\section{Chapter 3. Discussion and Outlook}

In this study I analyzed the physiological role of the adrenocortical circadian clock using total and conditional knockout mice lacking an essential clock gene Bmall. I showed that Bmall knockout mice develop hypocortisolism and have impaired hormonal and behavioral responses to acute stress. This impaired hormonal production is likely due to a defect in GC biosynthesis in the adrenal gland, rather than a result of systemic alterations, e.g. in HPA axis activity. In support, adrenal responsiveness of Bmall-deficient adrenals to ACTH stimulation in culture is drastically reduced and associated with down-regulation of adrenal transcripts involved in cholesterol transport and steroidogenesis such as

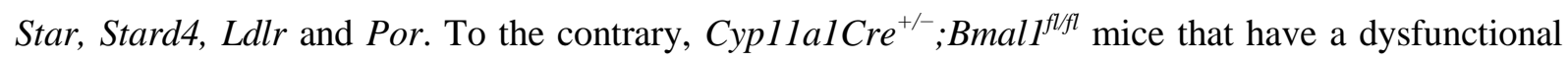
clock in steroid-producing cells only do not display substantial alterations in systemic levels of steroid hormones, including CORT, aldosterone and testosterone. Gene expression analysis of the adrenal transcripts that were found to be reduced in $\mathrm{Bmall}^{-/-}$adrenals demonstrate only minor changes in adrenal clock-deficient mice, although Star mRNA expression is consistently down-regulated. Interestingly, in accordance with previous findings, behavioral re-entrainment to a 6-hour advanced light-dark cycle occurs faster in the conditional mutant animals compared to controls. This suggests that the adrenocortical clock may contribute to the stabilization of circadian clock network rhythmicity, including the SCN, against perturbation by external Zeitgeber signals, though it seems not to be essential for the maintenance of normal GC rhythms under basal conditions.

In the Discussion I will summarize the information about potential physiological roles of adrenocortical clocks revealed by different genetic models and compare my findings with previous studies that used non-genetic tools to address similar questions. I will focus on three distinct aspects of adrenocortical clock biology: (I) maintenance of circadian GC rhythms; (II) regulation of hormonal and behavioral responses to stress; and (III) a role in circadian synchronization. Finally, I will suggest an updated view on the organization of the circadian timing system in mammals, considering unique properties of the adrenocortical clock.

\subsection{Conditional disruption of circadian clocks in the adrenal cortex: methodological issues}

Among in vivo loss-of-function approaches to study functional roles of peripheral circadian clocks are organ/tissue transplantations (for example, see (Kiessling et al., 2010; Oster et al., 2006b; Sun et al., 2006) or genetic conditional alterations of circadian clock function. The Cre-loxP gene targeting technology is a powerful tool to investigate gene function in a tissue-specific manner. It has been successfully used to evaluate roles of circadian clocks in a number of peripheral tissues, such as liver (Lamia et al., 2008), pancreas (Marcheva et al., 2010), adipose tissues (Paschos et al., 2012), and 
macrophages (Gibbs et al., 2012; Nguyen et al., 2013), and is now available for targeting the central clock in the SCN as an alternative to lesioning (Husse et al., 2011) as well as clocks in other brain areas (Mieda and Sakurai, 2011). In addition, in vivo genetic tools were used to alter the circadian clock in the brain and periphery including tissue-specific genetic knockdown (Son et al., 2008) and genetic "rescue" of a circadian clock, either by conditional inhibition of a transcriptional repressor (Kornmann et al., 2007) or by using the tetracycline transactivator system (McDearmon et al., 2006).

Is BMAL1 an optimal target for a genetic loss-of-function approach to study circadian tissue clocks? The advantages are that Bmall is the only non-redundant gene the deletion of which causes molecular clock disruption. It further is an important transcriptional activator, which allows easy identification of E-box-regulated (i.e. clock-controlled) genes after its knockout. However, Bmall ${ }^{-/-}$mice have the largest spectrum of described physiological abnormalities, compared to other circadian clock-deficient animals (e.g. (Alvarez et al., 2008; Bunger et al., 2005; Kondratov et al., 2006)). In addition, for many genes, not only the rhythmicity of transcription is lost, but also the expression is dramatically downregulated, or, in some cases, up-regulated, in the absence of Bmall, suggesting its pleiotropic effects (reviewed in (Yu and Weaver, 2011)). Clock deficient mice might be a good alternative to Bmal1 ${ }^{-/}$ animals in studying peripheral versus central effects of circadian clock disruption, since the CLOCK protein is necessary for circadian rhythms in peripheral tissues, at least in liver and lungs, but dispensable for behavioral rhythms in locomotor activity (Debruyne et al., 2006; DeBruyne et al., 2007b). Therefore one should take into account that a lack of Bmall or, likely, Clock genes results in a complex phenotype that combines the effects on both alterations in gene expression levels and loss of rhythmicity. Another potential approaches to study a role of circadian rhythms in peripheral tissues are behavioral arrhythmicity induced by constant light conditions, which impairs synchronization between SCN neurons, but leaves the molecular clockwork intact (Ohta et al., 2005), and dynamics of circadian clock re-entrainment after a shift of Zeitgeber cycle, which corresponds to a degree of the intrinsic robustness of a circadian clock in response to perturbation (Kiessling et al., 2010).

In this study I showed that circadian clock function is impaired in the adrenal cortex of the conditional mutant CypllalCre ${ }^{+/-}$;Bmall flfl mice, compared to CypllalCre ${ }^{+/-}$controls. Bmal1/BMAL1 levels are down-regulated to around $15 \%$ of the control levels, and rhythmic transcription of clock- and clock-controlled genes ( $D b p, N r l d l)$ is abolished. Since $D b p$ is a direct BMAL1/CLOCK target gene (Stratmann et al., 2012), its arrhythmicity clearly indicates a dysfunction of the BMAL1/CLOCK complex activity. According to published studies that used Bmall fl/fl animals for peripheral clock targeting, the most drastic effect of Bmall deletion is seen in the expression of Dbp and Nrldl, whereas Cry1 transcripts are up-regulated (e.g. (Gibbs et al., 2012; Lamia et al., 2008; Paschos et al., 2012)). Persistent Per2 rhythmicity can be driven by systemic cues even in the absence of intrinsic clock function (Hughes et al., 2012; Kornmann et al., 2007), which is in line with other studies (Lamia et al., 2008). Flattened rhythms or arrhythmic expression in mutant adrenals was also shown for some 
clock output genes such as Por, Mc2r and Prkce (Oster et al., 2006b; Park et al., 2013). This is consistent with previous reports showing that Por is regulated by the circadian clock in liver (Cho et al., 2012; Lamia et al., 2008). In conclusion, these data suggest that the circadian clock machinery is severely affected in the adrenal cortex of mutant mice. Although it is possible that in the $10-15 \%$ of residual adrenal cells that do not show recombination the circadian clock is still functional (Figure 10), the circadian gene expression data suggest that it is unlikely that these cells would significantly contribute to hormonal rhythms.

\subsection{The adrenocortical clock and hormonal rhythms}

I found that mice lacking Bmall in all tissues suffer from hypocortisolism, but are still capable of producing ACTH at levels resembling those in wild-type animals (section 2.1). Despite the fact that Bmall $^{-/-}$adrenals lack gross morphological defects, they do develop reduced adrenal responsiveness to ACTH stimulation in vitro and show impaired expression of transcripts involved in cholesterol transport and steroidogenesis (Star, Stard4, Ldlr and Por). It is worth noting that, similar to Bmal1 ${ }^{-/-}$ mice, reduced plasma and fecal CORT levels and impaired adrenal responses to ACTH in culture have previously been found in another genetic model of circadian disruption - Per2/Cryl mutant mice (Oster et al., 2006b). However potential alterations in the expression of steroidogenic genes in Per2/Cryl mice were not assessed.

In sharp contrast, plasma and fecal CORT levels in adrenocortical clock-deficient animals are not impaired in LD and DD conditions (Figure 12). This suggests that BMAL1 expression in other tissues outside the adrenal cortex provides an opportunity for compensation of the circadian clock defect in steroid-producing cells, which is not available in case of global Bmall disruption. One scenario for such compensation is that increased ACTH production would normalize GC production in spite of defective ACTH sensitivity. However, plasma ACTH levels in the mutant mice are unaltered (Figure 13). Another option is based on the contribution of the SCN-ANS pathway. In $\mathrm{Bmall}^{-/}$mice, compromised ANS signaling cannot compensate for a lack of adrenocortical ACTH sensitivity, whereas in the conditional knockout mice an intact ANS input may rescue ACTH sensitivity and hence restore basal rhythms of GC production in the absence of an adrenocortical circadian clock. In line, Mrap mRNA, which encodes a key adaptor molecule of the MC2R signaling pathway, is markedly over-expressed in the adrenal cortex of the conditional knockout mice. Since the adrenal medulla plays a key role in ANS-mediated regulation of adrenal sensitivity to ACTH (Ulrich-Lai et al., 2006) and is involved in setting the amplitude of circadian GC rhythms (Ikeda et al., 2013), an intact circadian clock in the adrenal medulla of the conditional mutant mice may have an important contribution to these interactions. But severely impaired circadian GC rhythms in mice with adrenocortical clock knockdown do not support this idea (Son et al., 2008). 
Some attempts have already been made to unveil a potential role of the adrenal clock in regulation of circadian GC rhythms. To inactivate the adrenal clock in vivo, Oster and co-workers transplanted pieces of adrenal glands from Per2/Cryl mice under the kidney capsule of wild-type animals (Oster et al., 2006b). Both under LD and DD conditions, the amplitudes of CORT secretion rhythms were dampened by $40-50 \%$ in the adrenal clock-deficient mice compared with control mice that received wild-type transplants. However, the same transplantation model used later by Kiessling and coworkers did not confirm down-regulation of GC excretion rhythms in adrenal clock-deficient mice in LD conditions (Kiessling et al., 2010). A potential explanation of this discrepancy is that the relatively short recovery period after surgery ( 2 weeks) was insufficient for functional re-innervation of the adrenal grafts (Oster et al., 2006b), whereas Kiessling et al. waited much longer (8 weeks) to ensure that transplanted tissues were completely re-innervated (Ulrich-Lai and Engeland, 2000). This again supports the view that SCN-ANS-mediated regulation of adrenal responsiveness to ACTH may play a critical role in maintaining a proper amplitude of circadian GC rhythms.

In another study, Son et al. used a genetic approach to investigate a role of the adrenal clock in generation of GC rhythms (Son et al., 2008). They generated a transgenic mouse line in which a part of the BMAL1 coding region was expressed in an antisense orientation under control of the ACTH receptor $(M c 2 r)$ promoter. The authors reported that circadian rhythms of adrenal GC synthesis are severely affected in the transgenic animals in constant darkness, although daily GC secretion in entrained conditions was not impaired. In addition, they showed flattened circadian rhythms of locomotor activity and down-regulated Perl mRNA expression in some peripheral organs, implying that the adrenal clock is essential for GC-mediated regulation of the circadian system under constant conditions (Son et al., 2008). However, the strong phenotype raises questions about the precision of the genetic targeting. One cannot exclude that Bmall expression in other tissues was affected due to a broader expression pattern of the construct in the transgenic mice (Haruyama et al., 2009). Indeed, we do not know whether HPA axis activity was affected in these mice, since ACTH levels were not measured. In addition, the ACTH receptor might be also expressed in the anterior pituitary and other brain structures, at least at certain developmental windows (Clark and Metherell, 2006; Nimura et al., 2006) which would interfere with clock regulation at upper levels of the HPA axis.

The adrenal cortex is the main, but not the only source of GC biosynthesis. Other tissues include intestinal epithelium, skin, thymus and few other tissues. Although we assume that Bmall deletion occurred in all steroidogenic cells of the mutant mice, including extra-adrenal GC-synthesizing cells, cell type-specific differences may provide a chance for compensation of GC production by elevated CORT synthesis outside the adrenal gland. Therefore, to exclude a potential contribution of nonadrenal sources of GCs to systemic CORT levels, intra-adrenal CORT content should be analyzed (Son et al., 2008). 
For conditional Bmall inactivation in GC-producing adrenal cells I used a Cre knock-in mouse line that also targets other steroidogenic cells including Leydig cells in male gonads and aldosteroneproducing cells in the adrenal zona glomerulosa. However, inactivation of Bmall at both locations has no apparent effects on hormonal production in these tissues, since plasma aldosterone levels are not significantly altered. Interestingly, plasma testosterone is moderately reduced at CT6 in the mutant mice, but fertility of the mutants does not differ substantially from controls (not shown). Therefore it is unlikely that inactivation of Bmall in other CRE-targeted cell types may significantly influence the function of GC-producing adrenocortical cells.

\subsection{The adrenocortical clock and stress responses}

This study revealed that hormonal and behavioral responses to acute stress are highly compromised in Bmall-deficient mice (see also Discussion in section 2.1). The reduced CORT response to 10-min forced swimming observed in the knockouts might be explained by impaired adrenal responsiveness to ACTH. Because the adrenocortical clock-deficient mice are capable of compensating the consequences of Bmall deficiency on basal GC regulation, it would be interesting to study whether the mutant animals also have normal hormonal responses to stress. Although reduced Star expression in the adrenocortical clock-deficient mice may potentially result in compromised adrenal function under stressed conditions (Figure 12), it is important to see whether induction of StAR expression in response to stress or ACTH stimulation is impaired in the mutants.

Expression of adrenal transcripts involved in the ACTH receptor signaling was also differentially regulated in complete and conditional knockout mice. Adrenals of $\mathrm{Bmall}^{-/-}$mice show up-regulated expression of the ACTH receptor gene $M c 2 r$, but unaltered expression of the accessory molecule Mrap, at CT12. ACD mice, to the contrary, have the opposite changes in the adrenal expression of $M c 2 r$ and Mrap genes. It is not clear so far how these differences affect the ACTH signaling and adrenal responsiveness to ACTH under homeostatic and stressed conditions.

Dysregulation of HPA axis upstream of the adrenal gland in Bmall-deficient mice may contribute to its sensitivity to negative GC feedback and therefore modulate GC responses to stress. Indeed, the results of the dexamethasone suppression test show that HPA axis of Bmall ${ }^{-/-}$mice is resistant to the negative GC feedback, which is in accordance with the finding that plasma ACTH levels are normal in spite of hypocortisolism. For instance, GR signaling in pituitary and/or PVN neurons may be altered in Bmall-deficient mice, since the circadian clock proteins BMAL1 and CRYs can interact with GR (Cheon et al., 2013b; Lamia et al., 2011; Nader et al., 2009). On the other hand, HPA axis is presumably less affected in animals with conditional Bmall knockout, compared with complete Bmall deficiency, since the circadian clocks in pituitary and hypothalamus are supposed to be functional in 
these mice. In line, Son and coworkers showed that mice with Bmall knockdown in adrenals do not differ from controls in GC response to 30-min restraint stress (Son et al., 2008).

Behavioral resistance to stress observed in Bmall-deficient mice may arise from impaired adrenal GC responses and/or altered GC sensitivity in the brain, as well as from Bmall deletion in brain areas that regulate mood- and reward-related behavior. For instance, under unstressed conditions $\mathrm{Bmall}^{-/-}$mice develop reduced sucrose preference indicating a depression-like phenotype, which is not related to GC responses to stress. Similarly, other studies support the view some behavioral abnormalities seen in Clock and Per 2 mutant mice are likely due to impaired circadian clock in the brain (Hampp et al., 2008; Roybal et al., 2007). On the other hand, this data clearly indicates that acute and sub-chronic stress has markedly reduced potential to alter behavior of $\mathrm{Bmall}^{-/}$mice in FST, TST and sucrose preference tests. Recent findings suggest that an increased amplitude of circadian GC rhythms has anxiolytic effect. Since the adrenocortical clock-deficient mice also reveal a clear trend in increased CORT release at the circadian peak of GC production, it would be interesting to test behavioral changes in these mice.

\subsection{The adrenocortical clock and the synchronization of circadian clocks}

In addition to a plethora of physiological and behavioral effects, GCs are systemic synchronizers of the circadian clock network, being able to directly induce the transcription of Per genes in various tissues. Indeed, a significant reduction in the number of rhythmic transcripts has been reported in the liver of adrenalectomized mice (Oishi et al., 2005). Importantly, it recently became clear that the central clock can also be a target of GCs. Sage et al. were first to demonstrate that adrenalectomized rats adapt their locomotor behavior faster in response to a shifted LD cycle, compared to sham operated animals (Sage et al., 2004). In an elegant study by Kiessling and colleagues, wild-type mice transplanted with Per2/Cryl adrenals also showed accelerated behavioral re-entrainment in a 6-hour jet lag experiment (Kiessling et al., 2010). Using my genetic model of adrenal clock deficiency, I confirmed that photic resynchronization of wheel-running behavior in response to a 6-hour phase advance occurs significantly faster in the mutant mice than in controls (Figure 15), while locomotor activity in LD and DD conditions is largely unaffected. Together, these data suggest that the adrenocortical clock decelerates the rate of behavioral resetting after shifting the LD cycle. This effect is likely mediated via modulation of the phase and/or amplitude of GC rhythms. This possibility is supported by the fact that pharmacological alteration of GC rhythms prior to a 6-hour shift of LD cycle affects the resetting speed of locomotor activity (Kiessling et al., 2010). Under normal LD conditions such a feedback control mechanism would stabilize clock phase by preventing unwanted resetting of the circadian system by sporadic light exposure, thus protecting it from Zeitgeber noise. 
The inhibitory effect of GCs on the behavioral re-entrainment during a jet lag can be conferred by different means (see (Kiessling et al., 2010; Sage et al., 2004) for detailed discussions). One possibility is that the SCN per se is the target of GC action. Since SCN neurons are devoid of significant levels of GR expression, GCs could influence the central clock indirectly, for instance, via the serotoninergic input from the midbrain raphe, which are known to express a high density of GR (Harfstrand et al., 1986). Indeed, Sage et al. showed that pharmacological suppression of serotoninergic signaling to the SCN in adrenalectomized rats prevented the modulatory effects of a restored corticosterone rhythm on photic synchronization of the locomotor activity (Sage et al., 2004). This indirect effect on the central clock may be mediated through changes in circadian coupling between the circadian clocks in individual SCN neurons (Yamaguchi et al., 2013). The second option is that GC oscillations modifies locomotor activity rhythms by an effect on brain areas involved in regulation of locomotion such as the striatum and the parietal cortex (Masubuchi et al., 2000). Finally, the light input pathway to the SCN may be regulated by GCs. Thus GCs could affect the SCN's photic sensitivity.

Another important aspect is a role of the adrenocortical clock in stabilization of circadian clocks in other peripheral tissues. Kiessling et al. found that clock gene resetting kinetics following a 6-hour LD phase advance differs significantly in various tissues (Kiessling et al., 2010). Therefore, the adrenocortical clock may be involved in the coordination of tissue clocks. Indeed, Pezük and coworkers recently demonstrated that adrenalectomy increases the rate of reentrainment after phase shifts in a number of peripheral tissues including liver, kidney and lung (Pezuk et al., 2012). Progenies of the adrenocortical clock-deficient mice cross-bred to circadian reporter Per2luc mice would be a valuable genetic model to assess this hypothesis.

\subsection{Towards a comprehensive understanding of the mammalian circadian organization: the adrenocortical clock and the stability of the circadian timing system}

In line with current knowledge, local circadian clocks contribute to physiological functions of a tissue through regulation of rhythmic transcription of genes relevant for execution of tissue-specific functions and regulation of sensitivity to systemic signals (Barclay et al., 2012; Dibner et al., 2010). GC-producing cells of the adrenal cortex are essential for coordination of physiological and behavioral responses to stressful stimuli. This study provides first evidence that Bmall-deficient mice develop deregulated gene expression locally in the adrenal gland, which leads to reduced adrenal sensitivity to ACTH and compromised GC production in response to acute stress.

On the other hand, the results of this study, together with previous findings, support a view that the adrenocortical clock has a unique position in the hierarchy of the circadian timing system (Figure 16). 
This is mainly explained by the fact that adrenal GCs, in addition to their role in stress responses, are important for the synchronization of circadian clocks in the periphery and in the brain.

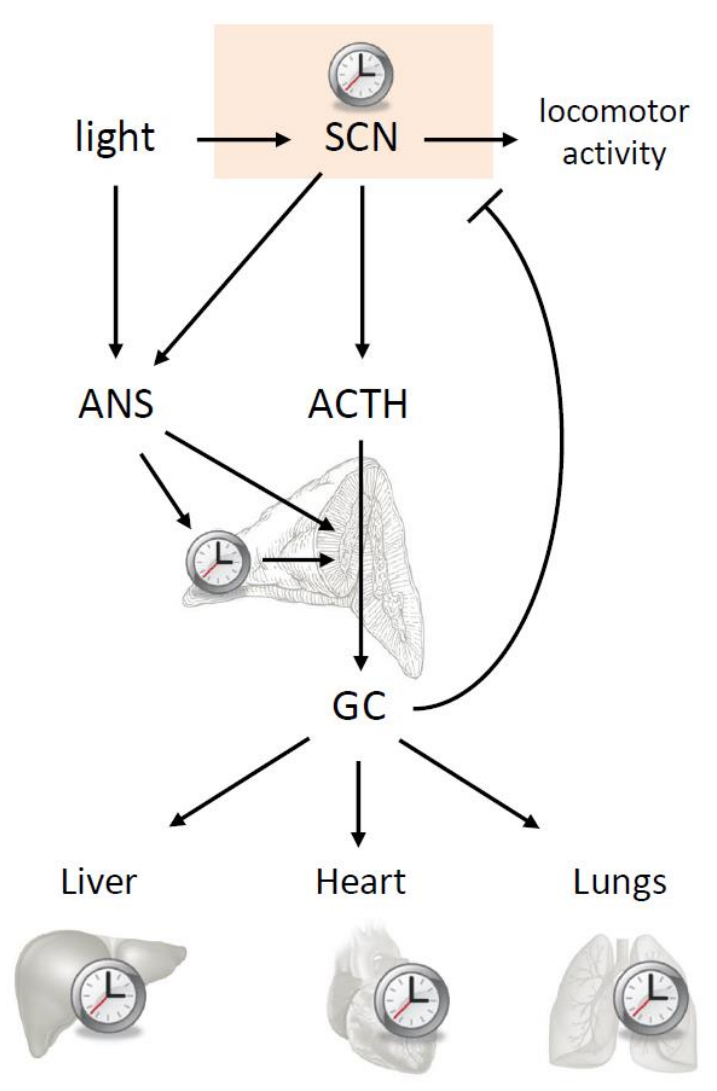

Figure 16. GCs regulate synchronization of the circadian system.
Under entrained conditions, rhythmic GC release is involved in daily resetting of peripheral clocks and also drives rhythmic transcription of some genes in various tissues. It seems that the adrenocortical clock plays only a minor role in the maintenance of daily GC rhythms under homeostatic conditions. However it is important for adapting to perturbed environmental conditions, such as stressful experiences or Zeigeber noise. Although exact mechanisms behind the effects of the adrenocortical clock on stress responses and stability of the circadian timing system are not fully understood, several possibilities come to mind (Figure 17). In general, I assume that upon perturbation (a shift of LD cycle or a stressful stimulus) the adrenocortical clock is involved in adjusting the level and/or the kinetics of GC synthesis (Figure 17A, lower part). Adrenocortical clock deficiency would therefore compromise GC responses to perturbation and result in reduced/delayed GC effects in target tissues (Figure 17B, lower part).

What are potential pathways that are under control of the adrenocortical clock? ACTH and autonomic innervation are two major inputs that regulate baseline adrenal steroidogenesis as well as adrenal GC responses to stimulation. Both pathways converge in the adrenal gland, since the ANS controls responsiveness of adrenocortical cells to ACTH (Figure 17A, arrow 1) (Engeland and Arnhold, 2005; Ulrich-Lai et al., 2006). However, their relative contribution depends on many factors, such as a type of stimulus. According to Okamura's study (Ishida et al., 2005), adrenal GC responses to light are mediated via SCN-ANS pathway, without stimulation of ACTH release, whereas both HPA axis and autonomic innervation contribute to stress-induced CORT production (Ulrich-Lai and Engeland, 2002). There are several possibilities how the adrenocortical clock might be involved in regulation of GC responses to light and stress, including direct regulation of ACTH sensitivity and steroidogenesis (Figure 17A, arrow 2) and gating of signals from ANS (Figure 17A, arrow 3). On the other hand, the adrenocortical clock per se and/or its output might be modulated by ANS (Figure 17A, arrow 4). 
Moreover, adrenal medulla is necessary for ANS-mediated regulation of adrenal sensitivity to ACTH (Engeland and Arnhold, 2005; Ishida et al., 2005), therefore a local clock in chromaffin cells (Lemos et al., 2006; Oster et al., 2006a) may add an extra dimension in this scheme. Finally, in addition of ACTH, other factors, e.g. IL-6, can directly affect adrenal GC production (Salas et al., 1990), and the adrenocortical clock might influence their action.

A
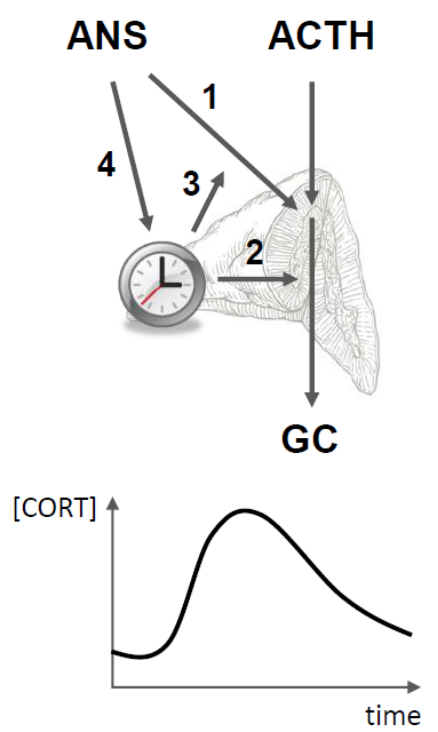

B

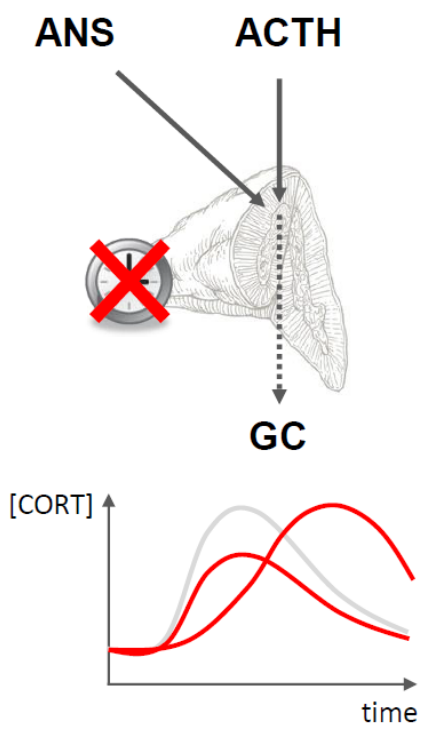

Figure 17. A role of the adrenocortical clock in organization of the circadian timing system. See the text for explanations. 


\subsection{Outlook}

The results of this study open a number of intriguing questions and ideas that would be interesting to address in future experiments.

- It is not clear how autonomic innervation of the adrenal gland affect the adrenocortical clock and adrenal sensitivity to ACTH and whether the clock itself regulates the ANS input. Adrenal denervation in $\mathrm{Bmall}^{-/}$and ACD mice would help reveal this.

- Although reduced GC production is well in line with altered behavioral responses to stress in Bmal1 $^{-/}$animals, a causative link between hormonal and behavioral changes has not been established in the study. Chronic restoration of daily GC oscillations in Bmal1-deficient mice by administering exogenous CORT may partially answer this question.

- When studying a role of the circadian clock in stress responses I tested a limited set of stress paradigms. Furthermore the kinetics of GC response to a stressor in vivo was not assessed. It would be interesting to study HPA axis activity and behavioral responses in Bmall $^{-/}$and ACD mice under conditions of chronic unpredictable stress or other stimuli that up-regulate GC production such as exercise.

- In addition to circadian rhythms, ultradian ACTH release is essential for pulsatile GC secretion. It would be important to clarify whether altered adrenal responsiveness to ACTH influence ultradian rhythms of GC production and, if so, what are consequences of these changes.

- Other paradigms for further analysis of circadian locomotor behavior (e.g. responses to constant light, phase responses to light pulses under free-run, T-cycles) of ACD mice should be used to fully understand the role of the adrenocortical clock in stabilization of the circadian system under perturbed light conditions. It also remains unclear how the GC-mediated feedback on the central clock works. Genetic or pharmacological manipulations with GR signaling in relevant brain areas would provide more understanding.

- Does the adrenocortical clock also regulate the robustness of circadian rhythms in peripheral tissues under conditions of unstable Zeitgeber input? To address this question, ACD mice harboring a circadian Per2luc reporter in their genome would be an elegant genetic model.

- This study shows that ACD mice lacking Bmall gene in all steroid-producing cells may also serve as a genetic model to study a circadian clock in testosterone-producing Leydig cells. 


\section{Chapter 4. Materials and Methods}

\subsection{Animal experiments}

\subsubsection{Animal housing and breeding}

All animal experiments in this study were carried out in compliance with the German Animal Welfare Act (TierSchG) and approved by the local state authorities. 3-4 months old mice of different genotypes were housed in transparent plastic cages, standard chow and water was provided ad libitum. Room temperature and humidity were held constant at $21{ }^{\circ} \mathrm{C}$ and $55 \%$, respectively, and animals were kept in a 12 hour light 12 hour dark cycle (LD; 300-400 lux).

\subsubsection{Tissue, blood and feces collection}

For adrenal slice culture experiments, analysis of gene expression and hormonal measurements in constant darkness conditions (DD), single-housed animals were entrained to LD cycle for at least one week and then released into DD. Tissues and blood were collected on the second day in DD (circadian time (CT) 0 in wild-type mice roughly corresponds to 36 hours after "lights off"). Animals were removed from their cages and immediately culled by cervical dislocation. Trunk blood was collected in Microvette 300 EDTA-coated tubes (Sarstedt), centrifuged at $2200 \mathrm{~g}$ for $20 \mathrm{~min}$ at $4{ }^{\circ} \mathrm{C}$, and plasma was frozen at $-80^{\circ} \mathrm{C}$ until use. For collection of fecal samples mice were housed in individual cages with metal grid floor. Before fecal collections, at least 4 days were provided to animals for adaptation to new housing conditions. Fecal samples were collected at 4-hour intervals and stored at $-80^{\circ} \mathrm{C}$ until extraction.

\subsubsection{Behavioral tests}

\section{Wheel running analysis}

Wheel running experiments were carried out as described previously (Jud et al., 2005; Kiessling et al., 2010). For all behavioral experiments male mice between 2 and 4 months of age were used. During recording, mice were kept in individual transparent plastic cages equipped with running wheels. Wheel revolutions were detected via a magnetic switch connected to a computer system. 12 cages were kept in one isolation chamber, in which lights were controlled via a computer system. Temperature (20 \pm $0.5{ }^{\circ} \mathrm{C}$ ) and humidity $(50-60 \%)$ were kept constant. Animals with different genotypes were always distributed equally between different isolation cabinets. Mice were provided standard chow food and 
water ad libitum. Locomotor activity parameters were analyzed using the ClockLab analysis software plug-in (Actimetrics) for MatLab (The Mathworks).

During LD conditions, overall and day-night wheel-running activity was measured. After entrainment to an LD12:12 cycle for at least 14 days lights were not turned on again at the following morning and animals were kept in constant darkness for two weeks to setermine the internal period $(\tau)$ of locomotor activity (the length of a subjective day determined by the time points of activity onset on two consecutive days). For a jet lag experiment, mice were entrained to LD12:12 cycle for at least 14 days and then lights were turned off $6 \mathrm{~h}$ earlier. The number of days needed by an animal to adapt to the new LD cycle was measured by determining the onsets of activity after the LD shift. Individual activity onsets before and after the shift were determined by using the ClockLab software.

\section{Forced swim test (FST)}

The test was performed at the end of the light phase (between Zeitgeber time (ZT) 10 and ZT12, ie, 10-12 hours after "lights on") as described in (Castagne et al., 2010), with minor modifications. Briefly, animals were placed for 6 minutes into a standard 3-L glass beaker filled with tap water (25 \pm $2^{\circ} \mathrm{C}$ ). Size of the beaker and level of water prevents mice from escape and does not allow reaching the bottom of the beaker. Every session was video recorded, and the duration of immobility over every minute of the 6-minute test was estimated using the CowLog open source software (http://cowlog. org) (Hanninen and Pastell, 2009).

\section{Repeated restraint stress}

Mice were exposed to confinement stress once daily for 2 hours between ZT10 and ZT12 for 7 consecutive days (Swiergiel et al., 2008) by keeping them in small transparent plastic restrainers (95 x $30 \times 32 \mathrm{~mm}$ ). Sucrose preference tests (SPTs; see below) were performed one day before the restraint period (baseline sucrose preference) and after 7 restraint sessions. Immobility behavior during a tail suspension test (TST) was assessed twice, one day before the beginning of the first and one day after the last restraint session.

\section{Tail suspension test (TST)}

TSTs were conducted according to a protocol described elsewhere (Castagne et al., 2010). Between ZT10 and ZT12, mice were suspended for 6 minutes by the tail on a horizontal bar at a height of 20$25 \mathrm{~cm}$ using a sticky paper tape. Every session was video recorded in the absence of the experimenter. The duration of immobility (i.e. passive hanging without movements) over the course of the 6-minute test was measured with assistance of the CowLog software. 


\section{Sucrose preference test (SPT)}

To estimate baseline sucrose preference (LeGates et al., 2012), mice were provided a choice between 2 bottles filled with $1 \%$ sucrose solution and tap water. To avoid positional preference, bottle positions were changed twice a day, in the middle of activity and rest phases, respectively. The bottles were weighed once a day (at the end of light phase) for 3 consecutive days, and sucrose and water intake were averaged. Occasional water leakage from individual bottles was thoroughly controlled. Sucrose preference after restraint stress was evaluated over a 24-hour period. To calculate the percentage of sucrose preference, the amount of consumed sucrose solution was divided by the amount of total liquid intake and multiplied by 100.

\subsubsection{Dexamethasone suppression test}

Mice received ip injection of dexamethasone solution ( $100 \mu \mathrm{g}$ per $\mathrm{kg}$ of body weight in $0.9 \%$ saline) at ZT8, and trunk blood was collected 6 hours later for CORT analysis. A control group was injected with $200 \mu \mathrm{L}$ of $0.9 \%$ saline solution. The selected dosage was previously shown to be efficient in suppressing CORT production down to about $20 \%$ compared with saline-injected controls (Bartolomucci et al., 2004).

\subsection{Histological methods}

\subsubsection{Hematoxylin-eosin staining and histological analysis of adrenal glands}

Isolated adrenal glands were removed from surrounding fat, weighed, fixed in $4 \%$ paraformaldehyde, dehydrated and embedded in paraffin. 8- $\mu \mathrm{m}$ adrenal sections were deparaffinized, rehydrated and stained with hematoxylin solution for $3 \mathrm{~min}$. To allow staining develop, the slides were rinsed with tap water and dipped several times into acid ethanol $(1 \mathrm{~mL}$ concentrated hydrochloric acid in $400 \mathrm{ml} 70 \%$ ethanol). Then sections were stained with eosin for $30 \mathrm{sec}$, dehydrated and mounted with a xylenebased mounting medium (Permount). To evaluate adrenal cortex-to-medulla ratio, cortical and medullar areas were measured from every specimen in at least 3 different sections close to the middle of the adrenal gland.

\subsubsection{Oil Red $O$ staining}

Lipid staining was performed on frozen adrenals obtained from untreated or repeatedly stressed mice (3 sequential 10-minute forced swim sessions with 30-minute rest intervals). Saturated (approximately $1 \%$ ) stock solution of Oil Red O (Sigma-Aldrich) in $99 \%$ isopropanol was prepared at least 2 days before staining. $1 \%$ dextrin solution (Sigma-Aldrich) was prepared the day before and stored at RT overnight, then was filtered through filter paper. The working solution was prepared by mixing $60 \mathrm{ml}$ 
of stock solution with $40 \mathrm{ml}$ of $1 \%$ dextrin and filtered through filter paper. Frozen sections were stained $20 \mathrm{~min}$ in Oil Red O staining solution, then washed in $60 \%$ isopropanol and mounted with glycerin jelly. Image analysis was performed with Image J software (National Institutes of Health, Bethesda, Maryland).

\subsubsection{Immunohistochemistry}

Adrenals were embedded in OCT medium (Tissue-Tek) and $10 \mu \mathrm{m}$ sections were cut using a cryotome (Leica). Sections were pre-treated with $3 \% \mathrm{H}_{2} \mathrm{O}_{2}$ in methanol for $10 \mathrm{~min}$ at room temperature and washed 3 times with deionized water. Antigen retrieval was performed in $0.01 \mathrm{M}$ sodium citrate $(\mathrm{pH}$ 6.0) for $2 \mathrm{~min}$ at room temperature. Then sections were boiled in the same solution for $5 \mathrm{~min}$ and cooled on ice for $20 \mathrm{~min}$. Next, slides were washed 2 times with TNT buffer $(0.1 \mathrm{M}$ Tris- $\mathrm{HCl} \mathrm{pH} 7.5$, $150 \mathrm{mM}$ sodium chloride, $0.05 \%$ TWEEN 20) for 2 min each. Blocking solution was applied for $2 \mathrm{~h}$ at room temperature (Vectastain kit) followed by anti-BMAL1 antibody solution (Novus Biologicals; 1:1,000 in TNT buffer) at $4{ }^{\circ} \mathrm{C}$ overnight. On the next day sections were washed in TNT buffer for 5 min, twice in PBS for 5 min and covered with secondary antibody (Vectastain kit) solution for 20 min. After 5-min PBS wash the ABC reagent (Vectastain kit) was applied for $30 \mathrm{~min}$. Then the ABC reagent was removed and slides were washed with PBS for $5 \mathrm{~min}$ and the DAB reagent (Vectastain kit) was applied until the color developed. Slides were mounted with Kaiser's glycerol gelatin (Merck).

\subsection{4. $\beta$-galactosidase staining of frozen tissues}

OCT (Tissue-Tek) embedded freshly frozen tissues were sectioned (10-16 $\mu \mathrm{m})$ and sections were fixed in $0.2 \%$ glutaraldehyde in 1x PBS for 5 min, washed in 1x PBS for 5 min and incubated over night with staining solution ( $5 \mathrm{mM}$ potassium ferricyanide, $5 \mathrm{mM}$ potassium ferrocyanide, $2 \mathrm{mM} \mathrm{MgCl}_{2}, 1$ $\mathrm{mg} / \mathrm{ml} \mathrm{X-Gal} \mathrm{solution,} 1 \mathrm{mM}$ phosphate, $150 \mathrm{mM} \mathrm{NaCl}$ ) at $30^{\circ} \mathrm{C}$. The next day, sections were washed in 1x PBS for $5 \mathrm{~min}$, counterstained with Nuclear fast Red solution (Sigma-Aldrich) for $5 \mathrm{~min}$ and dehydrated in progressively increasing ethanol concentrations (30\%, 70\%, 90\%, 100\% ethanol, each step $2 \mathrm{~min}$ ) followed by $2 \mathrm{x}$ incubation with xylene for $2 \mathrm{~min}$. Cover slips were mounted with DPX mounting medium (Sigma).

\subsection{Tissue culture experiments}

\section{Adrenal responsiveness to ACTH ex vivo}

Adrenal slice culture and adrenal ACTH stimulation ex vivo were performed as described previously (Oster et al., 2006b). 200- $\mu \mathrm{m}$ slices were precultured for 20 minutes on Millicell-CM membranes 
(Millipore Corp.) in DMEM (PAA Laboratories) supplemented with $0.1 \%$ dimethyl sulfoxide, $50 \mu \mathrm{M}$ 2-mercaptoethanol, $2 \%$ fetal bovine serum, and $0.12 \mathrm{mg} / \mathrm{mL}$ penicillin/ streptomycin at $37^{\circ} \mathrm{C}$ and $5 \%$ $\mathrm{CO}_{2}$. Slices were stimulated with $20 \mathrm{nM}$ ACTH and medium was collected immediately ( 0 minutes), 30 minutes, 90 minutes, and 210 minutes later. To study dose response to ACTH, adrenal slices were stimulated with 0,2 , and $20 \mathrm{nM} \mathrm{ACTH}$, and medium was collected 90 minutes later. Samples were stored at $-80^{\circ} \mathrm{C}$ until further processing.

\subsection{Hormonal measurements}

\subsubsection{Corticosterone extraction from mouse feces}

Fecal corticoid extraction was done according to a previously published protocol (Abraham et al., 2006), with few modifications. Fecal samples were collected in 4 hour intervals and stored at $-80^{\circ} \mathrm{C}$ until used. Samples were dried in a speed vac centrifuge over night and homogenized using $2.8 \mathrm{~mm}$ ceramic beads (Peqlab) in a OMNILAB bench homogenizer running 5x the following program: $3 x 30$ $\mathrm{sec}$ at $6800 \mathrm{rpm}$. Homogenized samples were dissolved in $10 \mathrm{ml}$ ethanol, boiled for $20 \mathrm{~min}$ in a water bath and centrifuged at $900 \mathrm{rcf}$ for $15 \mathrm{~min}$. Supernatant was poured into Petri dishes and pellets were re-extracted by adding $5 \mathrm{ml}$ ethanol and vortexing for $1 \mathrm{~min}$. After centrifugation at $900 \mathrm{rcf}$ for $15 \mathrm{~min}$ the second supernatant was added to the first supernatant in the Petri dish and samples were dried in an oven at $55^{\circ} \mathrm{C}$ for $45-90 \mathrm{~min}$. Dried extracts were dissolved in $1 \mathrm{ml}$ methanol and stored at $-80^{\circ} \mathrm{C}$ until use.

\subsubsection{Hormonal assays}

Corticosterone was measured from plasma or feces samples using the ImmuChem Double Antibody 125I-Radioimmunoassay Kit (MP Biomedicals) with the following modifications: for each reagent only $1 / 4$ of the volume was used, plasma samples were used at 1:200 dilutions, fecal samples were used at 1:5 dilutions. All samples were measured in duplicate and CORT concentrations were calculated from standard values by fitting a one-site competition function using Graph Pad software.

ACTH plasma concentrations were analyzed using the IMMULITE 1000 Immunoassay System (Siemens) at 1:2 to 1:4 dilutions. Since ACTH levels raise faster during stress, all efforts were taken to sacrifice animals within 1-2 minutes of handling.

Aldosterone and testosterone concentrations were measured using commercially available kits from Cayman and Immunotech, respectively, in accordance with manufacturer's instructions. 


\subsection{Molecular biological methods}

\subsubsection{Quantitative reverse transcription polymerase chain reaction ( $q R T$ - PCR)}

Quantitative analysis of mRNA levels was performed as described elsewhere (Barclay et al., 2013; Oster et al., 2006b). Total RNA was extracted from whole adrenal tissues using TRIzol Reagent (Life Technologies) according to the manufacturer's instructions. cDNA was synthesized using High Capacity cDNA Reverse Transcription kit (Life Technologies). qRT-PCR was performed on a C1000 Thermal Cycler and CFX96 Real-Time PCR Detection System (Bio-Rad Laboratories) with GoTaq qPCR Master Mix (Promega Corp.), and relative expression was assessed by comparison with Eeflal using the ddCT method (Oster et al., 2006a). Primer sequences are listed in section 2.1.

\subsubsection{Genotyping}

Tail snips were taken from 3-4 week old weaned mice and were lysed overnight at $55^{\circ} \mathrm{C}$ in $400 \mu \mathrm{l}$ tail extraction buffer (200 mM Tris-HCl, pH 8.0, 50 mM EDTA (pH 8.0), $100 \mathrm{mM} \mathrm{NaCl}, 1 \%$ SDS) with $10 \mu \mathrm{l}$ proteinase $\mathrm{K}(10 \mathrm{mg} / \mathrm{ml})$. Afterwards tissue debris was spun down at 13,000 rpm for $10 \mathrm{~min}$ and $200 \mu \mathrm{l}$ DNA-containing supernatant was mixed with $400 \mu \mathrm{l}$ of ice-cold $100 \%$ ethanol. Precipitated DNA was washed with $200 \mu \mathrm{l}$ of $70 \%$ ethanol and air-dried for 10 min. DNA was dissolved in TrisEDTA (10 mM Tris, pH 8.0, $1 \mathrm{mM}$ EDTA, pH 8.0). Genotyping PCR was carried out with a FlexCycler PCR machine (Analytik Jena).

Bmall knockout/flox genotyping was performed as previously described (Storch et al., 2007).

Reaction setup:

- $1 \mu \mathrm{l}$ DNA (approx. $200 \mathrm{ng} / \mu \mathrm{l}$ ),

- $2.5 \mu \mathrm{l}$ standard PCR buffer,

- $1 \mu \mathrm{l}$ dNTPs,

- $1 \mu \mathrm{lggCl}$,

- $1 \mu \mathrm{l}$ forward primer $1(10 \mu \mathrm{M})$,

- $1 \mu \mathrm{l}$ forward primer $2(10 \mu \mathrm{M})$,

- $2 \mu \mathrm{l}$ reverse primer $(10 \mu \mathrm{M})$,

- $0.5 \mu$ l Amplicon Taq polymerase,

- $16 \mu$ PCR-graded water.
Cycling program:

- $3 \min 94^{\circ} \mathrm{C}$,

- $30 \sec 94^{\circ} \mathrm{C}$,

- $1 \min 59^{\circ} \mathrm{C}, 35 \mathrm{x}$,

- $1 \min 72^{\circ} \mathrm{C}$,

- $5 \min 72^{\circ} \mathrm{C}$,

- keep at $4{ }^{\circ} \mathrm{C}$. 
Primers:

$\begin{array}{ll}\text { Bmal-flox-F } & \text { ACTGGAAGTAACTTTATCAAACTG } \\ \text { Bmal-flox-R } & \text { CTGACCAACTTGCTAACAATTA } \\ \text { Bmal-KO-R } & \text { CTCCTAACTTGGTTTTTGTCTGT }\end{array}$

Approximate DNA lengths of the amplified alleles are: wild-type- $420 \mathrm{bp}$, floxed-524 bp, knockout -726 bp.

Cypl1al-CreGFP genotyping was performed according to a protocol from the Jackson Laboratory (http://jaxmice.jax.org/strain/010988.html).

Reaction setup:

- $1 \mu \mathrm{l}$ DNA (approx. $200 \mathrm{ng} / \mu \mathrm{l}$ ),

- $2.5 \mu$ l standard PCR buffer,

- $1 \mu \mathrm{ldNTPs}$,

- $1 \mu \mathrm{l}$ forward primer $(10 \mu \mathrm{M})$,

- $1 \mu \mathrm{l}$ reverse primer $1(10 \mu \mathrm{M})$,

- $1 \mu \mathrm{l}$ reverse primer $2(10 \mu \mathrm{M})$,

- $0.3 \mu$ Amplicon Taq polymerase,

- $17.2 \mu \mathrm{l}$ PCR-graded water.

Primers:

$\begin{array}{ll}\text { Cyp11a1-F } & \text { GAGCTGCCTGCCAGTGTTTG } \\ \text { Cyp11a1-R1 } & \text { GGGGCCATACTTTTGGAAAC } \\ \text { GFP-R } & \text { GTCCAGCTCGACCAGGATGG }\end{array}$

Cycling program:

- $3 \min 94^{\circ} \mathrm{C}$,

- $30 \sec 94^{\circ} \mathrm{C}$,

- $1 \min 60^{\circ} \mathrm{C}, 35 \mathrm{x}$,

- $1 \min 72^{\circ} \mathrm{C}$,

- $10 \min 72^{\circ} \mathrm{C}$,

- keep at $4{ }^{\circ} \mathrm{C}$.

Approximate DNA lengths of the amplified alleles are: wild-type -488 bp, knock-in-296 bp.

\subsection{Statistical analysis}

Statistical analysis was performed using GraphPad Prism 5.0 (GraphPad). All data are represented as means \pm SEM. Whenever applicable, data distribution was tested for normality using the D'AgostinoPearson omnibus test. Two-group comparisons were done using unpaired $t$ tests. For multiple comparisons one- or two-way ANOVAs with Bonferroni post hoc test was used as indicated in the figure legends. Time course analyses were performed using repeated-measures two-way ANOVA. Rhythmicity of gene expression was tested using the Circwave software (developed by R.A. Hut, University of Groningen) and represented by fitting a sine curve using GraphPrism software. $P$ values below 0.05 were considered significant. 


\section{References}

Abe, K., Kroning, J., Greer, M.A., and Critchlow, V. (1979). Effects of destruction of the suprachiasmatic nuclei on the circadian rhythms in plasma corticosterone, body temperature, feeding and plasma thyrotropin. Neuroendocrinology 29, 119-131.

Abe, M., Herzog, E.D., Yamazaki, S., Straume, M., Tei, H., Sakaki, Y., Menaker, M., and Block, G.D. (2002). Circadian rhythms in isolated brain regions. J Neurosci 22, 350-356.

Abraham, D., Dallmann, R., Steinlechner, S., Albrecht, U., Eichele, G., and Oster, H. (2006). Restoration of circadian rhythmicity in circadian clock-deficient mice in constant light. J Biol Rhythms 21, 169-176.

Adam, T.C., and Epel, E.S. (2007). Stress, eating and the reward system. Physiol Behav 91, 449-458.

Agarwal, M.K., and Mirshahi, M. (1999). General overview of mineralocorticoid hormone action. Pharmacol Ther 84, 273-326.

Albrecht, U. (2013a). Circadian clocks and mood-related behaviors. Handb Exp Pharmacol 217, 227239.

Albrecht, U. (2013b). Circadian clocks and mood-related behaviors. Handb Exp Pharmacol, 227-239.

Albrecht, U., Sun, Z.S., Eichele, G., and Lee, C.C. (1997). A differential response of two putative mammalian circadian regulators, mper1 and mper2, to light. Cell 91, 1055-1064.

Alvarez, J.D., Hansen, A., Ord, T., Bebas, P., Chappell, P.E., Giebultowicz, J.M., Williams, C., Moss, S., and Sehgal, A. (2008). The circadian clock protein BMAL1 is necessary for fertility and proper testosterone production in mice. J Biol Rhythms 23, 26-36.

Ambrosi, B., Riva, E., and Faglia, G. (1985). Persistence of a circadian rhythmicity of glucocorticoid secretion in a patient with Cushing's syndrome: study before and after unilateral adrenalectomy. $\mathrm{J}$ Endocrinol Invest 8, 363-367.

Andrews, R.V., and Folk, G.E., Jr. (1964). Circadian Metabolic Patterns in Cultured Hamster Adrenal Glands. Comp Biochem Physiol 11, 393-409.

Angeles-Castellanos, M., Salgado-Delgado, R., Rodriguez, K., Buijs, R.M., and Escobar, C. (2010). The suprachiasmatic nucleus participates in food entrainment: a lesion study. Neuroscience 165, 11151126.

Aschoff, J., Fatranska, M., Giedke, H., Doerr, P., Stamm, D., and Wisser, H. (1971). Human circadian rhythms in continuous darkness: entrainment by social cues. Science 171, 213-215.

Asher, G., and Schibler, U. (2011). Crosstalk between components of circadian and metabolic cycles in mammals. Cell Metab 13, 125-137.

Balsalobre, A., Brown, S.A., Marcacci, L., Tronche, F., Kellendonk, C., Reichardt, H.M., Schutz, G., and Schibler, U. (2000). Resetting of circadian time in peripheral tissues by glucocorticoid signaling. Science 289, 2344-2347.

Balsalobre, A., Damiola, F., and Schibler, U. (1998). A serum shock induces circadian gene expression in mammalian tissue culture cells. Cell 93, 929-937. 
Barclay, J.L., Shostak, A., Leliavski, A., Tsang, A.H., Johren, O., Muller-Fielitz, H., Landgraf, D., Naujokat, N., van der Horst, G.T., and Oster, H. (2013). High fat diet-induced hyperinsulinemia and tissue-specific insulin resistance in Cry deficient mice. Am J Physiol Endocrinol Metab.

Barclay, J.L., Tsang, A.H., and Oster, H. (2012). Interaction of central and peripheral clocks in physiological regulation. Prog Brain Res 199, 163-181.

Bartolomucci, A., Pederzani, T., Sacerdote, P., Panerai, A.E., Parmigiani, S., and Palanza, P. (2004). Behavioral and physiological characterization of male mice under chronic psychosocial stress. Psychoneuroendocrinology 29, 899-910.

Bass, J., and Takahashi, J.S. (2010). Circadian integration of metabolism and energetics. Science 330, 1349-1354.

Bicknell, A.B. (2008). The tissue-specific processing of pro-opiomelanocortin. J Neuroendocrinol 20, 692-699.

Bittman, E.L., Doherty, L., Huang, L., and Paroskie, A. (2003). Period gene expression in mouse endocrine tissues. Am J Physiol Regul Integr Comp Physiol 285, R561-569.

Boggiano, M.M., Chandler, P.C., Viana, J.B., Oswald, K.D., Maldonado, C.R., and Wauford, P.K. (2005). Combined dieting and stress evoke exaggerated responses to opioids in binge-eating rats. Behav Neurosci 119, 1207-1214.

Boivin, D.B. (2000). Influence of sleep-wake and circadian rhythm disturbances in psychiatric disorders. J Psychiatry Neurosci 25, 446-458.

Bonfiglio, J.J., Inda, C., Refojo, D., Holsboer, F., Arzt, E., and Silberstein, S. (2011). The corticotropin-releasing hormone network and the hypothalamic-pituitary-adrenal axis: molecular and cellular mechanisms involved. Neuroendocrinology 94, 12-20.

Bornstein, S.R., Ehrhart-Bornstein, M., Scherbaum, W.A., Pfeiffer, E.F., and Holst, J.J. (1990). Effects of splanchnic nerve stimulation on the adrenal cortex may be mediated by chromaffin cells in a paracrine manner. Endocrinology 127, 900-906.

Bornstein, S.R., Engeland, W.C., Ehrhart-Bornstein, M., and Herman, J.P. (2008). Dissociation of ACTH and glucocorticoids. Trends Endocrinol Metab 19, 175-180.

Bouillon, D.J., and Berdanier, C.D. (1981). Effect of adrenalectomy on the diurnal variation in glycogen metabolism in starved-refed rats. J Nutr 111, 1462-1474.

Boyle, M.P., Brewer, J.A., Funatsu, M., Wozniak, D.F., Tsien, J.Z., Izumi, Y., and Muglia, L.J. (2005). Acquired deficit of forebrain glucocorticoid receptor produces depression-like changes in adrenal axis regulation and behavior. Proc Natl Acad Sci U S A 102, 473-478.

Bremner, J.D., Krystal, J.H., Charney, D.S., and Southwick, S.M. (1996). Neural mechanisms in dissociative amnesia for childhood abuse: relevance to the current controversy surrounding the "false memory syndrome". Am J Psychiatry 153, 71-82.

Brown, S.A., Zumbrunn, G., Fleury-Olela, F., Preitner, N., and Schibler, U. (2002). Rhythms of mammalian body temperature can sustain peripheral circadian clocks. Curr Biol 12, 1574-1583.

Buenafe, A.C. (2012). Diurnal rhythms are altered in a mouse model of multiple sclerosis. J Neuroimmunol 243, 12-17.

Buhr, E.D., and Takahashi, J.S. (2013). Molecular components of the Mammalian circadian clock. Handb Exp Pharmacol 217, 3-27. 
Buhr, E.D., Yoo, S.H., and Takahashi, J.S. (2010). Temperature as a universal resetting cue for mammalian circadian oscillators. Science 330, 379-385.

Buijs, R.M., Escobar, C., and Swaab, D.F. (2013). The circadian system and the balance of the autonomic nervous system. Handb Clin Neurol 117, 173-191.

Buijs, R.M., Kalsbeek, A., van der Woude, T.P., van Heerikhuize, J.J., and Shinn, S. (1993). Suprachiasmatic nucleus lesion increases corticosterone secretion. Am J Physiol 264, R1186-1192.

Buijs, R.M., Wortel, J., Van Heerikhuize, J.J., Feenstra, M.G., Ter Horst, G.J., Romijn, H.J., and Kalsbeek, A. (1999). Anatomical and functional demonstration of a multisynaptic suprachiasmatic nucleus adrenal (cortex) pathway. Eur J Neurosci 11, 1535-1544.

Bunger, M.K., Walisser, J.A., Sullivan, R., Manley, P.A., Moran, S.M., Kalscheur, V.L., Colman, R.J., and Bradfield, C.A. (2005). Progressive arthropathy in mice with a targeted disruption of the Mop3/Bmal-1 locus. Genesis 41, 122-132.

Bunger, M.K., Wilsbacher, L.D., Moran, S.M., Clendenin, C., Radcliffe, L.A., Hogenesch, J.B., Simon, M.C., Takahashi, J.S., and Bradfield, C.A. (2000). Mop3 is an essential component of the master circadian pacemaker in mammals. Cell 103, 1009-1017.

Bur, I.M., Zouaoui, S., Fontanaud, P., Coutry, N., Molino, F., Martin, A.O., Mollard, P., and Bonnefont, X. (2010). The comparison between circadian oscillators in mouse liver and pituitary gland reveals different integration of feeding and light schedules. PLoS One 5, e15316.

Buren, J., Bergstrom, S.A., Loh, E., Soderstrom, I., Olsson, T., and Mattsson, C. (2007). Hippocampal 11beta-hydroxysteroid dehydrogenase type 1 messenger ribonucleic acid expression has a diurnal variability that is lost in the obese Zucker rat. Endocrinology 148, 2716-2722.

Cailotto, C., La Fleur, S.E., Van Heijningen, C., Wortel, J., Kalsbeek, A., Feenstra, M., Pevet, P., and Buijs, R.M. (2005). The suprachiasmatic nucleus controls the daily variation of plasma glucose via the autonomic output to the liver: are the clock genes involved? Eur J Neurosci 22, 2531-2540.

Cailotto, C., van Heijningen, C., van der Vliet, J., van der Plasse, G., Habold, C., Kalsbeek, A., Pevet, P., and Buijs, R.M. (2008). Daily rhythms in metabolic liver enzymes and plasma glucose require a balance in the autonomic output to the liver. Endocrinology 149, 1914-1925.

Cascio, C.S., Shinsako, J., and Dallman, M.F. (1987). The suprachiasmatic nuclei stimulate evening ACTH secretion in the rat. Brain Res 423, 173-178.

Castagne, V., Moser, P., Roux, S., and Porsolt, R.D. (2010). Rodent models of depression: forced swim and tail suspension behavioral despair tests in rats and mice. Curr Protoc Pharmacol Chapter 5, Unit 58.

Castagne, V., Moser, P., Roux, S., and Porsolt, R.D. (2011). Rodent models of depression: forced swim and tail suspension behavioral despair tests in rats and mice. Curr Protoc Neurosci Chapter 8 , Unit 8 10A.

Chandola, T., Brunner, E., and Marmot, M. (2006). Chronic stress at work and the metabolic syndrome: prospective study. BMJ 332, 521-525.

Chapman, K., Holmes, M., and Seckl, J. (2013). 11beta-hydroxysteroid dehydrogenases: intracellular gate-keepers of tissue glucocorticoid action. Physiol Rev 93, 1139-1206.

Charmandari, E., Chrousos, G.P., Lambrou, G.I., Pavlaki, A., Koide, H., Ng, S.S., and Kino, T. (2011). Peripheral CLOCK regulates target-tissue glucocorticoid receptor transcriptional activity in a circadian fashion in man. PLoS One 6, e25612. 
Cheng, M.Y., Bullock, C.M., Li, C., Lee, A.G., Bermak, J.C., Belluzzi, J., Weaver, D.R., Leslie, F.M., and Zhou, Q.Y. (2002). Prokineticin 2 transmits the behavioural circadian rhythm of the suprachiasmatic nucleus. Nature 417, 405-410.

Cheon, S., Park, N., Cho, S., and Kim, K. (2013a). Glucocorticoid-mediated Period2 induction delays the phase of circadian rhythm. Nucleic Acids Res 41, 6161-6174.

Cheon, S., Park, N., Cho, S., and Kim, K. (2013b). Glucocorticoid-mediated Period2 induction delays the phase of circadian rhythm. Nucleic Acids Res.

Cho, H., Zhao, X., Hatori, M., Yu, R.T., Barish, G.D., Lam, M.T., Chong, L.W., DiTacchio, L., Atkins, A.R., Glass, C.K., et al. (2012). Regulation of circadian behaviour and metabolism by REVERB-alpha and REV-ERB-beta. Nature 485, 123-127.

Cho, K. (2001). Chronic 'jet lag' produces temporal lobe atrophy and spatial cognitive deficits. Nat Neurosci 4, 567-568.

Chowdrey, H.S., Larsen, P.J., Harbuz, M.S., Jessop, D.S., Aguilera, G., Eckland, D.J., and Lightman, S.L. (1995). Evidence for arginine vasopressin as the primary activator of the HPA axis during adjuvant-induced arthritis. Br J Pharmacol 116, 2417-2424.

Chu, A., Zhu, L., Blum, I.D., Mai, O., Leliavski, A., Fahrenkrug, J., Oster, H., Boehm, U., and Storch, K.F. (2013). Global but not gonadotrope-specific disruption of Bmal1 abolishes the luteinizing hormone surge without affecting ovulation. Endocrinology 154, 2924-2935.

Cizza, G., and Rother, K.I. (2012). Cortisol binding globulin: more than just a carrier? J Clin Endocrinol Metab 97, 77-80.

Clark, A.J., Chan, L.F., Chung, T.T., and Metherell, L.A. (2009). The genetics of familial glucocorticoid deficiency. Best Pract Res Clin Endocrinol Metab 23, 159-165.

Clark, A.J., and Metherell, L.A. (2006). Mechanisms of disease: the adrenocorticotropin receptor and disease. Nat Clin Pract Endocrinol Metab 2, 282-290.

Clark, A.J., and Weber, A. (1998). Adrenocorticotropin insensitivity syndromes. Endocr Rev 19, 828843.

Conway-Campbell, B.L., Sarabdjitsingh, R.A., McKenna, M.A., Pooley, J.R., Kershaw, Y.M., Meijer, O.C., De Kloet, E.R., and Lightman, S.L. (2010). Glucocorticoid ultradian rhythmicity directs cyclical gene pulsing of the clock gene period 1 in rat hippocampus. J Neuroendocrinol 22, 1093-1100.

Crofford, L.J., Kalogeras, K.T., Mastorakos, G., Magiakou, M.A., Wells, J., Kanik, K.S., Gold, P.W., Chrousos, G.P., and Wilder, R.L. (1997). Circadian relationships between interleukin (IL)-6 and hypothalamic-pituitary-adrenal axis hormones: failure of IL-6 to cause sustained hypercortisolism in patients with early untreated rheumatoid arthritis. J Clin Endocrinol Metab 82, 1279-1283.

Curtis, A.M., Cheng, Y., Kapoor, S., Reilly, D., Price, T.S., and Fitzgerald, G.A. (2007). Circadian variation of blood pressure and the vascular response to asynchronous stress. Proc Natl Acad Sci U S A $104,3450-3455$.

Czeh, B., Michaelis, T., Watanabe, T., Frahm, J., de Biurrun, G., van Kampen, M., Bartolomucci, A., and Fuchs, E. (2001). Stress-induced changes in cerebral metabolites, hippocampal volume, and cell proliferation are prevented by antidepressant treatment with tianeptine. Proc Natl Acad Sci U S A 98, 12796-12801.

Daan, S. (2010). A history of chronobiological concepts. In The Circadian Clock, U. Albrecht, ed. (Springer), pp. 1-36. 
Dallman, M.F., Akana, S.F., Strack, A.M., Scribner, K.S., Pecoraro, N., La Fleur, S.E., Houshyar, H., and Gomez, F. (2004). Chronic stress-induced effects of corticosterone on brain: direct and indirect. Ann N Y Acad Sci 1018, 141-150.

Dallman, M.F., Engeland, W.C., Rose, J.C., Wilkinson, C.W., Shinsako, J., and Siedenburg, F. (1978). Nycthemeral rhythm in adrenal responsiveness to ACTH. Am J Physiol 235, R210-218.

Dallman, M.F., Pecoraro, N., Akana, S.F., La Fleur, S.E., Gomez, F., Houshyar, H., Bell, M.E., Bhatnagar, S., Laugero, K.D., and Manalo, S. (2003). Chronic stress and obesity: a new view of "comfort food". Proc Natl Acad Sci U S A 100, 11696-11701.

Dallmann, R., Touma, C., Palme, R., Albrecht, U., and Steinlechner, S. (2006). Impaired daily glucocorticoid rhythm in Per1 ( Brd ) mice. J Comp Physiol A Neuroethol Sens Neural Behav Physiol $192,769-775$.

Damiola, F., Le Minh, N., Preitner, N., Kornmann, B., Fleury-Olela, F., and Schibler, U. (2000). Restricted feeding uncouples circadian oscillators in peripheral tissues from the central pacemaker in the suprachiasmatic nucleus. Genes Dev 14, 2950-2961.

Darlington, D.N., Neves, R.B., Ha, T., Chew, G., and Dallman, M.F. (1990). Fed, but not fasted, adrenalectomized rats survive the stress of hemorrhage and hypovolemia. Endocrinology 127, 759765 .

Datson, N.A., van der Perk, J., de Kloet, E.R., and Vreugdenhil, E. (2001). Identification of corticosteroid-responsive genes in rat hippocampus using serial analysis of gene expression. Eur $\mathbf{J}$ Neurosci 14, 675-689.

de Kloet, E.R., Joels, M., and Holsboer, F. (2005). Stress and the brain: from adaptation to disease. Nat Rev Neurosci 6, 463-475.

de la Iglesia, H.O., Meyer, J., Carpino, A., Jr., and Schwartz, W.J. (2000). Antiphase oscillation of the left and right suprachiasmatic nuclei. Science 290, 799-801.

Debruyne, J.P., Noton, E., Lambert, C.M., Maywood, E.S., Weaver, D.R., and Reppert, S.M. (2006). A clock shock: mouse CLOCK is not required for circadian oscillator function. Neuron 50, 465-477.

DeBruyne, J.P., Weaver, D.R., and Reppert, S.M. (2007a). CLOCK and NPAS2 have overlapping roles in the suprachiasmatic circadian clock. Nat Neurosci 10, 543-545.

DeBruyne, J.P., Weaver, D.R., and Reppert, S.M. (2007b). Peripheral circadian oscillators require CLOCK. Curr Biol 17, R538-539.

Deroche-Gamonet, V., Sillaber, I., Aouizerate, B., Izawa, R., Jaber, M., Ghozland, S., Kellendonk, C., Le Moal, M., Spanagel, R., Schutz, G., et al. (2003). The glucocorticoid receptor as a potential target to reduce cocaine abuse. J Neurosci 23, 4785-4790.

Dibner, C., Schibler, U., and Albrecht, U. (2010). The mammalian circadian timing system: organization and coordination of central and peripheral clocks. Annu Rev Physiol 72, 517-549.

Dickmeis, T. (2009). Glucocorticoids and the circadian clock. J Endocrinol 200, 3-22.

Dickmeis, T., and Foulkes, N.S. (2011). Glucocorticoids and circadian clock control of cell proliferation: at the interface between three dynamic systems. Mol Cell Endocrinol 331, 11-22.

Dickmeis, T., Lahiri, K., Nica, G., Vallone, D., Santoriello, C., Neumann, C.J., Hammerschmidt, M., and Foulkes, N.S. (2007). Glucocorticoids play a key role in circadian cell cycle rhythms. PLoS Biol 5, e78. 
Dijkstra, G., de Rooij, D.G., de Jong, F.H., and van den Hurk, R. (1996a). Effect of hypothyroidism on ovarian follicular development, granulosa cell proliferation and peripheral hormone levels in the prepubertal rat. Eur J Endocrinol 134, 649-654.

Dijkstra, I., Binnekade, R., and Tilders, F.J. (1996b). Diurnal variation in resting levels of corticosterone is not mediated by variation in adrenal responsiveness to adrenocorticotropin but involves splanchnic nerve integrity. Endocrinology 137, 540-547.

Dimitrov, S., Benedict, C., Heutling, D., Westermann, J., Born, J., and Lange, T. (2009). Cortisol and epinephrine control opposing circadian rhythms in T cell subsets. Blood 113, 5134-5143.

Doherty, C.J., and Kay, S.A. (2010). Circadian control of global gene expression patterns. Annu Rev Genet 44, 419-444.

Doi, M., Hirayama, J., and Sassone-Corsi, P. (2006). Circadian regulator CLOCK is a histone acetyltransferase. Cell 125, 497-508.

Doi, M., Takahashi, Y., Komatsu, R., Yamazaki, F., Yamada, H., Haraguchi, S., Emoto, N., Okuno, Y., Tsujimoto, G., Kanematsu, A., et al. (2010). Salt-sensitive hypertension in circadian clockdeficient Cry-null mice involves dysregulated adrenal Hsd3b6. Nat Med 16, 67-74.

Dunn, J., Scheving, L., and Millet, P. (1972). Circadian variation in stress-evoked increases in plasma corticosterone. Am J Physiol 223, 402-406.

Edwards, A.V., and Jones, C.T. (1987). The effect of splanchnic nerve section on the sensitivity of the adrenal cortex to adrenocorticotrophin in the calf. J Physiol 390, 23-31.

Edwards, A.V., Jones, C.T., and Bloom, S.R. (1986). Reduced adrenal cortical sensitivity to ACTH in lambs with cut splanchnic nerves. J Endocrinol 110, 81-85.

Ehrhart-Bornstein, M., Hinson, J.P., Bornstein, S.R., Scherbaum, W.A., and Vinson, G.P. (1998). Intraadrenal interactions in the regulation of adrenocortical steroidogenesis. Endocr Rev 19, 101-143.

Engeland, W.C. (2013). Sensitization of endocrine organs to anterior pituitary hormones by the autonomic nervous system. Handb Clin Neurol 117, 37-44.

Engeland, W.C., and Arnhold, M.M. (2005). Neural circuitry in the regulation of adrenal corticosterone rhythmicity. Endocrine 28, 325-332.

Engeland, W.C., and Gann, D.S. (1989). Splanchnic nerve stimulation modulates steroid secretion in hypophysectomized dogs. Neuroendocrinology 50, 124-131.

Engler, D., Pham, T., Liu, J.P., Fullerton, M.J., Clarke, I.J., and Funder, J.W. (1990). Studies of the regulation of the hypothalamic-pituitary-adrenal axis in sheep with hypothalamic-pituitary disconnection. II. Evidence for in vivo ultradian hypersecretion of proopiomelanocortin peptides by the isolated anterior and intermediate pituitary. Endocrinology 127, 1956-1966.

Epel, E., Lapidus, R., McEwen, B., and Brownell, K. (2001). Stress may add bite to appetite in women: a laboratory study of stress-induced cortisol and eating behavior. Psychoneuroendocrinology $26,37-49$.

Evanson, N.K., Tasker, J.G., Hill, M.N., Hillard, C.J., and Herman, J.P. (2010). Fast feedback inhibition of the HPA axis by glucocorticoids is mediated by endocannabinoid signaling. Endocrinology 151, 4811-4819. 
Fahrenkrug, J., Hannibal, J., and Georg, B. (2008). Diurnal rhythmicity of the canonical clock genes Per1, Per2 and Bmal1 in the rat adrenal gland is unaltered after hypophysectomy. J Neuroendocrinol 20, 323-329.

Filipski, E., Delaunay, F., King, V.M., Wu, M.W., Claustrat, B., Grechez-Cassiau, A., Guettier, C., Hastings, M.H., and Francis, L. (2004). Effects of chronic jet lag on tumor progression in mice. Cancer Res 64, 7879-7885.

Funder, J.W., Pearce, P.T., Smith, R., and Smith, A.I. (1988). Mineralocorticoid action: target tissue specificity is enzyme, not receptor, mediated. Science 242, 583-585.

Fustin, J.M., Doi, M., Yamaguchi, Y., Hida, H., Nishimura, S., Yoshida, M., Isagawa, T., Morioka, M.S., Kakeya, H., Manabe, I., et al. (2013). RNA-methylation-dependent RNA processing controls the speed of the circadian clock. Cell 155, 793-806.

Gamaleia, N.F., Shishko, E.D., and Chernyi, A.P. (1988). [Preservation of the circadian rhythm of human lymphocytes under in vitro conditions]. Biull Eksp Biol Med 106, 598-600.

Garcia, A., Steiner, B., Kronenberg, G., Bick-Sander, A., and Kempermann, G. (2004). Agedependent expression of glucocorticoid- and mineralocorticoid receptors on neural precursor cell populations in the adult murine hippocampus. Aging Cell 3, 363-371.

Gibbs, J.E., Blaikley, J., Beesley, S., Matthews, L., Simpson, K.D., Boyce, S.H., Farrow, S.N., Else, K.J., Singh, D., Ray, D.W., et al. (2012). The nuclear receptor REV-ERBalpha mediates circadian regulation of innate immunity through selective regulation of inflammatory cytokines. Proc Natl Acad Sci U S A 109, 582-587.

Gibbs, J.E., and Ray, D.W. (2013). The role of the circadian clock in rheumatoid arthritis. Arthritis Res Ther 15, 205.

Girard, C., Barbier, O., Veilleux, G., El-Alfy, M., and Belanger, A. (2003). Human uridine diphosphate-glucuronosyltransferase UGT2B7 conjugates mineralocorticoid and glucocorticoid metabolites. Endocrinology 144, 2659-2668.

Girotti, M., Weinberg, M.S., and Spencer, R.L. (2009). Diurnal expression of functional and clockrelated genes throughout the rat HPA axis: system-wide shifts in response to a restricted feeding schedule. Am J Physiol Endocrinol Metab 296, E888-897.

Goeders, N.E. (2002). Stress and cocaine addiction. J Pharmacol Exp Ther 301, 785-789.

Goldbeter, A. (2002). Computational approaches to cellular rhythms. Nature 420, 238-245.

Golombek, D.A., and Rosenstein, R.E. (2010). Physiology of circadian entrainment. Physiol Rev 90, 1063-1102.

Groeneweg, F.L., Karst, H., de Kloet, E.R., and Joels, M. (2011). Rapid non-genomic effects of corticosteroids and their role in the central stress response. J Endocrinol 209, 153-167.

Guilding, C., and Piggins, H.D. (2007). Challenging the omnipotence of the suprachiasmatic timekeeper: are circadian oscillators present throughout the mammalian brain? Eur J Neurosci 25, 3195-3216.

Guler, A.D., Ecker, J.L., Lall, G.S., Haq, S., Altimus, C.M., Liao, H.W., Barnard, A.R., Cahill, H., Badea, T.C., Zhao, H., et al. (2008). Melanopsin cells are the principal conduits for rod-cone input to non-image-forming vision. Nature 453, 102-105. 
Hampp, G., Ripperger, J.A., Houben, T., Schmutz, I., Blex, C., Perreau-Lenz, S., Brunk, I., Spanagel, R., Ahnert-Hilger, G., Meijer, J.H., et al. (2008). Regulation of monoamine oxidase A by circadianclock components implies clock influence on mood. Curr Biol 18, 678-683.

Hanninen, L., and Pastell, M. (2009). CowLog: open-source software for coding behaviors from digital video. Behav Res Methods 41, 472-476.

Harfstrand, A., Fuxe, K., Cintra, A., Agnati, L.F., Zini, I., Wikstrom, A.C., Okret, S., Yu, Z.Y., Goldstein, M., Steinbusch, H., et al. (1986). Glucocorticoid receptor immunoreactivity in monoaminergic neurons of rat brain. Proc Natl Acad Sci U S A 83, 9779-9783.

Harkness, J.A., Richter, M.B., Panayi, G.S., Van de Pette, K., Unger, A., Pownall, R., and Geddawi, M. (1982). Circadian variation in disease activity in rheumatoid arthritis. Br Med J (Clin Res Ed) 284, 551-554.

Haruyama, N., Cho, A., and Kulkarni, A.B. (2009). Overview: engineering transgenic constructs and mice. Curr Protoc Cell Biol Chapter 19, Unit 1910.

Henley, D.E., Leendertz, J.A., Russell, G.M., Wood, S.A., Taheri, S., Woltersdorf, W.W., and Lightman, S.L. (2009a). Development of an automated blood sampling system for use in humans. J Med Eng Technol 33, 199-208.

Henley, D.E., and Lightman, S.L. (2011). New insights into corticosteroid-binding globulin and glucocorticoid delivery. Neuroscience 180, 1-8.

Henley, D.E., Russell, G.M., Douthwaite, J.A., Wood, S.A., Buchanan, F., Gibson, R., Woltersdorf, W.W., Catterall, J.R., and Lightman, S.L. (2009b). Hypothalamic-pituitary-adrenal axis activation in obstructive sleep apnea: the effect of continuous positive airway pressure therapy. J Clin Endocrinol Metab 94, 4234-4242.

Hensleigh, E., and Pritchard, L.M. (2013). Glucocorticoid receptor expression and sub-cellular localization in dopamine neurons of the rat midbrain. Neurosci Lett 556, 191-195.

Herman, J.P., McKlveen, J.M., Solomon, M.B., Carvalho-Netto, E., and Myers, B. (2012). Neural regulation of the stress response: glucocorticoid feedback mechanisms. Braz J Med Biol Res 45, 292298.

Hinz, B., and Hirschelmann, R. (2000). Rapid non-genomic feedback effects of glucocorticoids on CRF-induced ACTH secretion in rats. Pharm Res 17, 1273-1277.

Holsboer, F. (2000). The corticosteroid receptor hypothesis of depression. Neuropsychopharmacology $23,477-501$.

Hsu, B.R., and Kuhn, R.W. (1988). The role of the adrenal in generating the diurnal variation in circulating levels of corticosteroid-binding globulin in the rat. Endocrinology 122, 421-426.

Hu, J., Zhang, Z., Shen, W.J., and Azhar, S. (2010). Cellular cholesterol delivery, intracellular processing and utilization for biosynthesis of steroid hormones. Nutr Metab (Lond) 7, 47.

Hu, M.C., Hsu, N.C., El Hadj, N.B., Pai, C.I., Chu, H.P., Wang, C.K., and Chung, B.C. (2002). Steroid deficiency syndromes in mice with targeted disruption of Cyp11a1. Mol Endocrinol 16, $1943-$ 1950.

Huang, N., Chelliah, Y., Shan, Y., Taylor, C.A., Yoo, S.H., Partch, C., Green, C.B., Zhang, H., and Takahashi, J.S. (2012). Crystal structure of the heterodimeric CLOCK:BMAL1 transcriptional activator complex. Science 337, 189-194. 
Hughes, M.E., DiTacchio, L., Hayes, K.R., Vollmers, C., Pulivarthy, S., Baggs, J.E., Panda, S., and Hogenesch, J.B. (2009). Harmonics of circadian gene transcription in mammals. PLoS Genet 5, e1000442.

Hughes, M.E., Hong, H.K., Chong, J.L., Indacochea, A.A., Lee, S.S., Han, M., Takahashi, J.S., and Hogenesch, J.B. (2012). Brain-specific rescue of Clock reveals system-driven transcriptional rhythms in peripheral tissue. PLoS Genet 8, e1002835.

Husse, J., Zhou, X., Shostak, A., Oster, H., and Eichele, G. (2011). Synaptotagmin10-Cre, a driver to disrupt clock genes in the SCN. J Biol Rhythms 26, 379-389.

Ikeda, Y., Kumagai, H., Skach, A., Sato, M., and Yanagisawa, M. (2013). Modulation of Circadian Glucocorticoid Oscillation via Adrenal Opioid-CXCR7 Signaling Alters Emotional Behavior. Cell $155,1323-1336$.

Ishida, A., Mutoh, T., Ueyama, T., Bando, H., Masubuchi, S., Nakahara, D., Tsujimoto, G., and Okamura, H. (2005). Light activates the adrenal gland: timing of gene expression and glucocorticoid release. Cell Metab 2, 297-307.

Jarcho, M.R., Slavich, G.M., Tylova-Stein, H., Wolkowitz, O.M., and Burke, H.M. (2013). Dysregulated diurnal cortisol pattern is associated with glucocorticoid resistance in women with major depressive disorder. Biol Psychol 93, 150-158.

Jiang, W.G., Li, S.X., Liu, J.F., Sun, Y., Zhou, S.J., Zhu, W.L., Shi, J., and Lu, L. (2013). Hippocampal CLOCK protein participates in the persistence of depressive-like behavior induced by chronic unpredictable stress. Psychopharmacology (Berl) 227, 79-92.

Jiang, W.G., Li, S.X., Zhou, S.J., Sun, Y., Shi, J., and Lu, L. (2011). Chronic unpredictable stress induces a reversible change of PER2 rhythm in the suprachiasmatic nucleus. Brain Res 1399, 25-32.

Joels, M., and Baram, T.Z. (2009). The neuro-symphony of stress. Nat Rev Neurosci 10, 459-466.

John, S., Sabo, P.J., Johnson, T.A., Sung, M.H., Biddie, S.C., Lightman, S.L., Voss, T.C., Davis, S.R., Meltzer, P.S., Stamatoyannopoulos, J.A., et al. (2008). Interaction of the glucocorticoid receptor with the chromatin landscape. Mol Cell 29, 611-624.

Jud, C., Schmutz, I., Hampp, G., Oster, H., and Albrecht, U. (2005). A guideline for analyzing circadian wheel-running behavior in rodents under different lighting conditions. Biol Proced Online 7, 101-116.

Kalsbeek, A., Buijs, R.M., van Heerikhuize, J.J., Arts, M., and van der Woude, T.P. (1992). Vasopressin-containing neurons of the suprachiasmatic nuclei inhibit corticosterone release. Brain Res $580,62-67$.

Kalsbeek, A., van der Spek, R., Lei, J., Endert, E., Buijs, R.M., and Fliers, E. (2012). Circadian rhythms in the hypothalamo-pituitary-adrenal (HPA) axis. Mol Cell Endocrinol 349, 20-29.

Kalsbeek, A., van der Vliet, J., and Buijs, R.M. (1996a). Decrease of endogenous vasopressin release necessary for expression of the circadian rise in plasma corticosterone: a reverse microdialysis study. $\mathrm{J}$ Neuroendocrinol 8, 299-307.

Kalsbeek, A., van Heerikhuize, J.J., Wortel, J., and Buijs, R.M. (1996b). A diurnal rhythm of stimulatory input to the hypothalamo-pituitary-adrenal system as revealed by timed intrahypothalamic administration of the vasopressin V1 antagonist. J Neurosci 16, 5555-5565.

Kaneko, M., Hiroshige, T., Shinsako, J., and Dallman, M.F. (1980). Diurnal changes in amplification of hormone rhythms in the adrenocortical system. Am J Physiol 239, R309-316. 
Kaneko, M., Kaneko, K., Shinsako, J., and Dallman, M.F. (1981). Adrenal sensitivity to adrenocorticotropin varies diurnally. Endocrinology 109, 70-75.

Kant, G.J., Mougey, E.H., and Meyerhoff, J.L. (1986). Diurnal variation in neuroendocrine response to stress in rats: plasma ACTH, beta-endorphin, beta-LPH, corticosterone, prolactin and pituitary cyclic AMP responses. Neuroendocrinology 43, 383-390.

Karssen, A.M., Meijer, O.C., van der Sandt, I.C., Lucassen, P.J., de Lange, E.C., de Boer, A.G., and de Kloet, E.R. (2001). Multidrug resistance P-glycoprotein hampers the access of cortisol but not of corticosterone to mouse and human brain. Endocrinology 142, 2686-2694.

Karst, H., Berger, S., Turiault, M., Tronche, F., Schutz, G., and Joels, M. (2005). Mineralocorticoid receptors are indispensable for nongenomic modulation of hippocampal glutamate transmission by corticosterone. Proc Natl Acad Sci U S A 102, 19204-19207.

Kiessling, S., Eichele, G., and Oster, H. (2010). Adrenal glucocorticoids have a key role in circadian resynchronization in a mouse model of jet lag. J Clin Invest 120, 2600-2609.

Kil, I.S., Lee, S.K., Ryu, K.W., Woo, H.A., Hu, M.C., Bae, S.H., and Rhee, S.G. (2012). Feedback control of adrenal steroidogenesis via $\mathrm{H} 2 \mathrm{O} 2$-dependent, reversible inactivation of peroxiredoxin III in mitochondria. Mol Cell 46, 584-594.

Kim, J.J., and Diamond, D.M. (2002). The stressed hippocampus, synaptic plasticity and lost memories. Nat Rev Neurosci 3, 453-462.

Kinoshita, C., Miyazaki, K., and Ishida, N. (2012). Chronic stress affects PERIOD2 expression through glycogen synthase kinase-3beta phosphorylation in the central clock. Neuroreport 23, 98-102.

Kirwan, J.R., Clarke, L., Hunt, L.P., Perry, M.G., Straub, R.H., and Jessop, D.S. (2010). Effect of novel therapeutic glucocorticoids on circadian rhythms of hormones and cytokines in rheumatoid arthritis. Ann N Y Acad Sci 1193, 127-133.

Kjolhede, E.A., Gustafsson, P., and Nelson, N. (2013). Overweight and obese children have lower cortisol levels than normal weight children. Acta Paediatr.

Ko, C.H., Yamada, Y.R., Welsh, D.K., Buhr, E.D., Liu, A.C., Zhang, E.E., Ralph, M.R., Kay, S.A., Forger, D.B., and Takahashi, J.S. (2010). Emergence of noise-induced oscillations in the central circadian pacemaker. PLoS Biol 8, e1000513.

Kohsaka, A., Laposky, A.D., Ramsey, K.M., Estrada, C., Joshu, C., Kobayashi, Y., Turek, F.W., and Bass, J. (2007). High-fat diet disrupts behavioral and molecular circadian rhythms in mice. Cell Metab $6,414-421$.

Koike, N., Yoo, S.H., Huang, H.C., Kumar, V., Lee, C., Kim, T.K., and Takahashi, J.S. (2012). Transcriptional architecture and chromatin landscape of the core circadian clock in mammals. Science $338,349-354$.

Kole, M.H., Costoli, T., Koolhaas, J.M., and Fuchs, E. (2004). Bidirectional shift in the cornu ammonis 3 pyramidal dendritic organization following brief stress. Neuroscience 125, 337-347.

Kollet, O., Vagima, Y., D'Uva, G., Golan, K., Canaani, J., Itkin, T., Gur-Cohen, S., Kalinkovich, A., Caglio, G., Medaglia, C., et al. (2013). Physiologic corticosterone oscillations regulate murine hematopoietic stem/progenitor cell proliferation and CXCL12 expression by bone marrow stromal progenitors. Leukemia 27, 2006-2015. 
Kondo, T., Strayer, C.A., Kulkarni, R.D., Taylor, W., Ishiura, M., Golden, S.S., and Johnson, C.H. (1993). Circadian rhythms in prokaryotes: luciferase as a reporter of circadian gene expression in cyanobacteria. Proc Natl Acad Sci U S A 90, 5672-5676.

Kondratov, R.V., Kondratova, A.A., Gorbacheva, V.Y., Vykhovanets, O.V., and Antoch, M.P. (2006). Early aging and age-related pathologies in mice deficient in BMAL1, the core componentof the circadian clock. Genes Dev 20, 1868-1873.

Konopka, R.J., and Benzer, S. (1971). Clock mutants of Drosophila melanogaster. Proc Natl Acad Sci U S A 68, 2112-2116.

Koob, G.F. (2008). A role for brain stress systems in addiction. Neuron 59, 11-34.

Kornmann, B., Schaad, O., Bujard, H., Takahashi, J.S., and Schibler, U. (2007). System-driven and oscillator-dependent circadian transcription in mice with a conditionally active liver clock. PLoS Biol $5, \mathrm{e} 34$.

Kovac, J., Husse, J., and Oster, H. (2009). A time to fast, a time to feast: the crosstalk between metabolism and the circadian clock. Mol Cells 28, 75-80.

Kramer, A., Yang, F.C., Snodgrass, P., Li, X., Scammell, T.E., Davis, F.C., and Weitz, C.J. (2001). Regulation of daily locomotor activity and sleep by hypothalamic EGF receptor signaling. Science 294, 2511-2515.

Kraves, S., and Weitz, C.J. (2006). A role for cardiotrophin-like cytokine in the circadian control of mammalian locomotor activity. Nat Neurosci 9, 212-219.

Krieger, D.T., Hauser, H., and Krey, L.C. (1977). Suprachiasmatic nuclear lesions do not abolish food-shifted circadian adrenal and temperature rhythmicity. Science 197, 398-399.

Kvetnansky, R., Sabban, E.L., and Palkovits, M. (2009). Catecholaminergic systems in stress: structural and molecular genetic approaches. Physiol Rev 89, 535-606.

la Fleur, S.E., Kalsbeek, A., Wortel, J., Fekkes, M.L., and Buijs, R.M. (2001). A daily rhythm in glucose tolerance: a role for the suprachiasmatic nucleus. Diabetes 50, 1237-1243.

Ladd, C.O., Huot, R.L., Thrivikraman, K.V., Nemeroff, C.B., and Plotsky, P.M. (2004). Long-term adaptations in glucocorticoid receptor and mineralocorticoid receptor mRNA and negative feedback on the hypothalamo-pituitary-adrenal axis following neonatal maternal separation. Biol Psychiatry 55, 367-375.

Lamia, K.A., Papp, S.J., Yu, R.T., Barish, G.D., Uhlenhaut, N.H., Jonker, J.W., Downes, M., and Evans, R.M. (2011). Cryptochromes mediate rhythmic repression of the glucocorticoid receptor. Nature 480, 552-556.

Lamia, K.A., Storch, K.F., and Weitz, C.J. (2008). Physiological significance of a peripheral tissue circadian clock. Proc Natl Acad Sci U S A 105, 15172-15177.

Landgraf, D., Shostak, A., and Oster, H. (2012). Clock genes and sleep. Pflugers Arch 463, 3-14.

Laryea, G., Schutz, G., and Muglia, L.J. (2013). Disrupting hypothalamic glucocorticoid receptors causes HPA axis hyperactivity and excess adiposity. Mol Endocrinol 27, 1655-1665.

Le Minh, N., Damiola, F., Tronche, F., Schutz, G., and Schibler, U. (2001). Glucocorticoid hormones inhibit food-induced phase-shifting of peripheral circadian oscillators. EMBO J 20, 7128-7136. 
Lechner, O., Dietrich, H., Oliveira dos Santos, A., Wiegers, G.J., Schwarz, S., Harbutz, M., Herold, M., and Wick, G. (2000). Altered circadian rhythms of the stress hormone and melatonin response in lupus-prone MRL/MP-fas(Ipr) mice. J Autoimmun 14, 325-333.

Lee, J.E., and Edery, I. (2008). Circadian regulation in the ability of Drosophila to combat pathogenic infections. Curr Biol 18, 195-199.

LeGates, T.A., Altimus, C.M., Wang, H., Lee, H.K., Yang, S., Zhao, H., Kirkwood, A., Weber, E.T., and Hattar, S. (2012). Aberrant light directly impairs mood and learning through melanopsinexpressing neurons. Nature 491, 594-598.

Lemos, D.R., Downs, J.L., and Urbanski, H.F. (2006). Twenty-four-hour rhythmic gene expression in the rhesus macaque adrenal gland. Mol Endocrinol 20, 1164-1176.

Levi, F., La Vecchia, C., Negri, E., and Lucchini, F. (1997). Declining cancer mortality in European Union. Lancet 349, 508-509.

Levi, F., and Schibler, U. (2007). Circadian rhythms: mechanisms and therapeutic implications. Annu Rev Pharmacol Toxicol 47, 593-628.

Levine, S. (1957). Infantile experience and resistance to physiological stress. Science 126, 405.

Li, S.X., Liu, L.J., Xu, L.Z., Gao, L., Wang, X.F., Zhang, J.T., and Lu, L. (2013). Diurnal alterations in circadian genes and peptides in major depressive disorder before and after escitalopram treatment. Psychoneuroendocrinology 38, 2789-2799.

Lightman, S.L., and Conway-Campbell, B.L. (2010). The crucial role of pulsatile activity of the HPA axis for continuous dynamic equilibration. Nat Rev Neurosci 11, 710-718.

Lilley, T.R., Wotus, C., Taylor, D., Lee, J.M., and de la Iglesia, H.O. (2012). Circadian regulation of cortisol release in behaviorally split golden hamsters. Endocrinology 153, 732-738.

Lincoln, G.A., Clarke, I.J., Hut, R.A., and Hazlerigg, D.G. (2006). Characterizing a mammalian circannual pacemaker. Science 314, 1941-1944.

Liston, C., Cichon, J.M., Jeanneteau, F., Jia, Z., Chao, M.V., and Gan, W.B. (2013). Circadian glucocorticoid oscillations promote learning-dependent synapse formation and maintenance. Nat Neurosci 16, 698-705.

Liu, A.C., Tran, H.G., Zhang, E.E., Priest, A.A., Welsh, D.K., and Kay, S.A. (2008). Redundant function of REV-ERBalpha and beta and non-essential role for Bmall cycling in transcriptional regulation of intracellular circadian rhythms. PLoS Genet 4, e1000023.

Liu, A.C., Welsh, D.K., Ko, C.H., Tran, H.G., Zhang, E.E., Priest, A.A., Buhr, E.D., Singer, O., Meeker, K., Verma, I.M., et al. (2007). Intercellular coupling confers robustness against mutations in the SCN circadian clock network. Cell 129, 605-616.

Liu, D., Diorio, J., Tannenbaum, B., Caldji, C., Francis, D., Freedman, A., Sharma, S., Pearson, D., Plotsky, P.M., and Meaney, M.J. (1997). Maternal care, hippocampal glucocorticoid receptors, and hypothalamic-pituitary-adrenal responses to stress. Science 277, 1659-1662.

Livingstone, D.E., Jones, G.C., Smith, K., Jamieson, P.M., Andrew, R., Kenyon, C.J., and Walker, B.R. (2000). Understanding the role of glucocorticoids in obesity: tissue-specific alterations of corticosterone metabolism in obese Zucker rats. Endocrinology 141, 560-563.

Loh, D.H., Abad, C., Colwell, C.S., and Waschek, J.A. (2008). Vasoactive intestinal peptide is critical for circadian regulation of glucocorticoids. Neuroendocrinology 88, 246-255. 
Ma, X.M., Levy, A., and Lightman, S.L. (1997). Emergence of an isolated arginine vasopressin (AVP) response to stress after repeated restraint: a study of both AVP and corticotropin-releasing hormone messenger ribonucleic acid (RNA) and heteronuclear RNA. Endocrinology 138, 4351-4357.

Magarinos, A.M., McEwen, B.S., Flugge, G., and Fuchs, E. (1996). Chronic psychosocial stress causes apical dendritic atrophy of hippocampal CA3 pyramidal neurons in subordinate tree shrews. J Neurosci 16, 3534-3540.

Magrini, A., Pietroiusti, A., Coppeta, L., Babbucci, A., Barnaba, E., Papadia, C., Iannaccone, U., Boscolo, P., Bergamaschi, E., and Bergamaschi, A. (2006). Shift work and autoimmune thyroid disorders. Int J Immunopathol Pharmacol 19, 31-36.

Marchant, E.G., and Mistlberger, R.E. (1997). Anticipation and entrainment to feeding time in intact and SCN-ablated C57BL/6j mice. Brain Res 765, 273-282.

Marcheva, B., Ramsey, K.M., Buhr, E.D., Kobayashi, Y., Su, H., Ko, C.H., Ivanova, G., Omura, C., Mo, S., Vitaterna, M.H., et al. (2010). Disruption of the clock components CLOCK and BMAL1 leads to hypoinsulinaemia and diabetes. Nature 466, 627-631.

Marzolla, V., Armani, A., Zennaro, M.C., Cinti, F., Mammi, C., Fabbri, A., Rosano, G.M., and Caprio, M. (2012). The role of the mineralocorticoid receptor in adipocyte biology and fat metabolism. Mol Cell Endocrinol 350, 281-288.

Mason, H., Colao, A., Blume-Peytavi, U., Rice, S., Qureshi, A., Pellatt, L., Orio, F., and Atkin, S.L. (2008). Polycystic ovary syndrome (PCOS) trilogy: a translational and clinical review. Clin Endocrinol (Oxf) 69, 831-844.

Masubuchi, S., Honma, S., Abe, H., Ishizaki, K., Namihira, M., Ikeda, M., and Honma, K. (2000). Clock genes outside the suprachiasmatic nucleus involved in manifestation of locomotor activity rhythm in rats. Eur J Neurosci 12, 4206-4214.

Mavroudis, P.D., Scheff, J.D., Calvano, S.E., Lowry, S.F., and Androulakis, I.P. (2012). Entrainment of peripheral clock genes by cortisol. Physiol Genomics 44, 607-621.

McDearmon, E.L., Patel, K.N., Ko, C.H., Walisser, J.A., Schook, A.C., Chong, J.L., Wilsbacher, L.D., Song, E.J., Hong, H.K., Bradfield, C.A., et al. (2006). Dissecting the functions of the mammalian clock protein BMAL1 by tissue-specific rescue in mice. Science 314, 1304-1308.

McEwen, B.S., and Gianaros, P.J. (2010). Central role of the brain in stress and adaptation: links to socioeconomic status, health, and disease. Ann N Y Acad Sci 1186, 190-222.

McEwen, B.S., and Sapolsky, R.M. (1995). Stress and cognitive function. Curr Opin Neurobiol 5, 205-216.

Meier, A.H. (1976). Daily variation in concentration of plasma corticosteroid in hypophysectomized rats. Endocrinology 98, 1475-1479.

Meijer, O.C., de Lange, E.C., Breimer, D.D., de Boer, A.G., Workel, J.O., and de Kloet, E.R. (1998). Penetration of dexamethasone into brain glucocorticoid targets is enhanced in mdr1A P-glycoprotein knockout mice. Endocrinology 139, 1789-1793.

Menaker, M., and Eskin, A. (1966). Entrainment of circadian rhythms by sound in Passer domesticus. Science $154,1579-1581$.

Mergenhagen, D., and Mergenhagen, E. (1987). The biological clock of Chlamydomonas reinhardii in space. Eur J Cell Biol 43, 203-207. 
Meyer-Bernstein, E.L., Jetton, A.E., Matsumoto, S.I., Markuns, J.F., Lehman, M.N., and Bittman, E.L. (1999). Effects of suprachiasmatic transplants on circadian rhythms of neuroendocrine function in golden hamsters. Endocrinology 140, 207-218.

Mieda, M., and Sakurai, T. (2011). Bmal1 in the nervous system is essential for normal adaptation of circadian locomotor activity and food intake to periodic feeding. J Neurosci 31, 15391-15396.

Mistlberger, R.E. (2011). Neurobiology of food anticipatory circadian rhythms. Physiol Behav 104, 535-545.

Mistlberger, R.E., and Skene, D.J. (2005). Nonphotic entrainment in humans? J Biol Rhythms 20, 339-352.

Mittag, M., Kiaulehn, S., and Johnson, C.H. (2005). The circadian clock in Chlamydomonas reinhardtii. What is it for? What is it similar to? Plant Physiol 137, 399-409.

Mondragon-Palomino, O., Danino, T., Selimkhanov, J., Tsimring, L., and Hasty, J. (2011). Entrainment of a population of synthetic genetic oscillators. Science 333, 1315-1319.

Montagnese, S., Middleton, B., Mani, A.R., Skene, D.J., and Morgan, M.Y. (2011). Changes in the 24-h plasma cortisol rhythm in patients with cirrhosis. J Hepatol 54, 588-590; author reply 590-581.

Moore, H.P., Andresen, J.M., Eaton, B.A., Grabe, M., Haugwitz, M., Wu, M.M., and Machen, T.E. (2002). Biosynthesis and secretion of pituitary hormones: dynamics and regulation. Arch Physiol Biochem 110, 16-25.

Moore, R.Y., and Eichler, V.B. (1972). Loss of a circadian adrenal corticosterone rhythm following suprachiasmatic lesions in the rat. Brain Res 42, 201-206.

Morley, J.E., Levine, A.S., and Rowland, N.E. (1983). Minireview. Stress induced eating. Life Sci 32, 2169-2182.

Mormont, M.C., and Levi, F. (1997). Circadian-system alterations during cancer processes: a review. Int J Cancer 70, 241-247.

Muglia, L.J., Jacobson, L., Weninger, S.C., Luedke, C.E., Bae, D.S., Jeong, K.H., and Majzoub, J.A. (1997). Impaired diurnal adrenal rhythmicity restored by constant infusion of corticotropin-releasing hormone in corticotropin-releasing hormone-deficient mice. J Clin Invest 99, 2923-2929.

Mukherji, A., Kobiita, A., Ye, T., and Chambon, P. (2013). Homeostasis in intestinal epithelium is orchestrated by the circadian clock and microbiota cues transduced by TLRs. Cell 153, 812-827.

Musiek, E.S., and Fitzgerald, G.A. (2013). Molecular clocks in pharmacology. Handb Exp Pharmacol, 243-260.

Nader, N., Chrousos, G.P., and Kino, T. (2009). Circadian rhythm transcription factor CLOCK regulates the transcriptional activity of the glucocorticoid receptor by acetylating its hinge region lysine cluster: potential physiological implications. FASEB J 23, 1572-1583.

Nader, N., Chrousos, G.P., and Kino, T. (2010). Interactions of the circadian CLOCK system and the HPA axis. Trends Endocrinol Metab 21, 277-286.

Nakamura, Y., Harama, D., Shimokawa, N., Hara, M., Suzuki, R., Tahara, Y., Ishimaru, K., Katoh, R., Okumura, K., Ogawa, H., et al. (2011). Circadian clock gene Period2 regulates a time-of-daydependent variation in cutaneous anaphylactic reaction. J Allergy Clin Immunol 127, 1038-1045 e1031-1033. 
Nguyen, A.D., and Conley, A.J. (2008). Adrenal androgens in humans and nonhuman primates: production, zonation and regulation. Endocr Dev 13, 33-54.

Nguyen, K.D., Fentress, S.J., Qiu, Y., Yun, K., Cox, J.S., and Chawla, A. (2013). Circadian gene Bmal1 regulates diurnal oscillations of Ly6C(hi) inflammatory monocytes. Science 341, 1483-1488.

Nimura, M., Udagawa, J., Hatta, T., Hashimoto, R., and Otani, H. (2006). Spatial and temporal patterns of expression of melanocortin type 2 and 5 receptors in the fetal mouse tissues and organs. Anat Embryol (Berl) 211, 109-117.

Nishiyama, M., Makino, S., Suemaru, S., Nanamiya, W., Asaba, K., Kaneda, T., Mimoto, T., Nishioka, T., Takao, T., and Hashimoto, K. (2000). Glucocorticoid effects on the diurnal rhythm of circulating leptin levels. Horm Res 54, 69-73.

Noguchi, T., Ikeda, M., Ohmiya, Y., and Nakajima, Y. (2012). A dual-color luciferase assay system reveals circadian resetting of cultured fibroblasts by co-cultured adrenal glands. PLoS One 7, e37093.

Ohta, H., Yamazaki, S., and McMahon, D.G. (2005). Constant light desynchronizes mammalian clock neurons. Nat Neurosci 8, 267-269.

Oishi, K., Amagai, N., Shirai, H., Kadota, K., Ohkura, N., and Ishida, N. (2005). Genome-wide expression analysis reveals 100 adrenal gland-dependent circadian genes in the mouse liver. DNA Res $12,191-202$.

Oitzl, M.S., and de Kloet, E.R. (1992). Selective corticosteroid antagonists modulate specific aspects of spatial orientation learning. Behav Neurosci 106, 62-71.

Oitzl, M.S., Reichardt, H.M., Joels, M., and de Kloet, E.R. (2001). Point mutation in the mouse glucocorticoid receptor preventing DNA binding impairs spatial memory. Proc Natl Acad Sci U S A 98, 12790-12795.

Olijslagers, J.E., de Kloet, E.R., Elgersma, Y., van Woerden, G.M., Joels, M., and Karst, H. (2008). Rapid changes in hippocampal CA1 pyramidal cell function via pre- as well as postsynaptic membrane mineralocorticoid receptors. Eur J Neurosci 27, 2542-2550.

Oliver, P.L., Sobczyk, M.V., Maywood, E.S., Edwards, B., Lee, S., Livieratos, A., Oster, H., Butler, R., Godinho, S.I., Wulff, K., et al. (2012). Disrupted circadian rhythms in a mouse model of schizophrenia. Curr Biol 22, 314-319.

Olsen, N.J., Fang, V.S., and DeGroot, L.J. (1978). Cushing's syndrome due to adrenal adenoma with persistent diurnal cortisol secretory rhythm. Metabolism 27, 695-700.

Opherk, C., Tronche, F., Kellendonk, C., Kohlmuller, D., Schulze, A., Schmid, W., and Schutz, G. (2004). Inactivation of the glucocorticoid receptor in hepatocytes leads to fasting hypoglycemia and ameliorates hyperglycemia in streptozotocin-induced diabetes mellitus. Mol Endocrinol 18, 13461353.

Ortiz-Tudela, E., Mteyrek, A., Ballesta, A., Innominato, P.F., and Levi, F. (2013). Cancer chronotherapeutics: experimental, theoretical, and clinical aspects. Handb Exp Pharmacol, 261-288.

Oster, H. (2006). The genetic basis of circadian behavior. Genes Brain Behav 5 Suppl 2, 73-79.

Oster, H., Damerow, S., Hut, R.A., and Eichele, G. (2006a). Transcriptional profiling in the adrenal gland reveals circadian regulation of hormone biosynthesis genes and nucleosome assembly genes. $\mathbf{J}$ Biol Rhythms 21, 350-361. 
Oster, H., Damerow, S., Kiessling, S., Jakubcakova, V., Abraham, D., Tian, J., Hoffmann, M.W., and Eichele, G. (2006b). The circadian rhythm of glucocorticoids is regulated by a gating mechanism residing in the adrenal cortical clock. Cell Metab 4, 163-173.

Ottenweller, J.E., and Meier, A.H. (1982). Adrenal innervation may be an extrapituitary mechanism able to regulate adrenocortical rhythmicity in rats. Endocrinology 111, 1334-1338.

Papadimitriou, A., and Priftis, K.N. (2009). Regulation of the hypothalamic-pituitary-adrenal axis. Neuroimmunomodulation 16, 265-271.

Park, S.Y., Walker, J.J., Johnson, N.W., Zhao, Z., Lightman, S.L., and Spiga, F. (2013). Constant light disrupts the circadian rhythm of steroidogenic proteins in the rat adrenal gland. Mol Cell Endocrinol $371,114-123$.

Parylak, S.L., Koob, G.F., and Zorrilla, E.P. (2011). The dark side of food addiction. Physiol Behav 104, 149-156.

Paschos, G.K., Ibrahim, S., Song, W.L., Kunieda, T., Grant, G., Reyes, T.M., Bradfield, C.A., Vaughan, C.H., Eiden, M., Masoodi, M., et al. (2012). Obesity in mice with adipocyte-specific deletion of clock component Arntl. Nat Med 18, 1768-1777.

Pevet, P., and Challet, E. (2011). Melatonin: both master clock output and internal time-giver in the circadian clocks network. J Physiol Paris 105, 170-182.

Pezuk, P., Mohawk, J.A., Wang, L.A., and Menaker, M. (2012). Glucocorticoids as entraining signals for peripheral circadian oscillators. Endocrinology 153, 4775-4783.

Pijlman, F.T., Wolterink, G., and Van Ree, J.M. (2003). Physical and emotional stress have differential effects on preference for saccharine and open field behaviour in rats. Behav Brain Res 139, 131-138.

Pirich, K., and Vierhapper, H. (1988). 24-hour serum concentration profile of cortisol in patients with Cushing's disease. Exp Clin Endocrinol 92, 275-279.

Pittendrigh, C.S. (1954). On Temperature Independence in the Clock System Controlling Emergence Time in Drosophila. Proc Natl Acad Sci U S A 40, 1018-1029.

Plihal, W., and Born, J. (1999). Memory consolidation in human sleep depends on inhibition of glucocorticoid release. Neuroreport 10, 2741-2747.

Preitner, N., Damiola, F., Lopez-Molina, L., Zakany, J., Duboule, D., Albrecht, U., and Schibler, U. (2002). The orphan nuclear receptor REV-ERBalpha controls circadian transcription within the positive limb of the mammalian circadian oscillator. Cell 110, 251-260.

Puttonen, S., Oksanen, T., Vahtera, J., Pentti, J., Virtanen, M., Salo, P., and Kivimaki, M. (2010). Is shift work a risk factor for rheumatoid arthritis? The Finnish Public Sector study. Ann Rheum Dis 69, 779-780.

Quax, R.A., Manenschijn, L., Koper, J.W., Hazes, J.M., Lamberts, S.W., van Rossum, E.F., and Feelders, R.A. (2013). Glucocorticoid sensitivity in health and disease. Nat Rev Endocrinol 9, 670686.

Quirarte, G.L., Roozendaal, B., and McGaugh, J.L. (1997). Glucocorticoid enhancement of memory storage involves noradrenergic activation in the basolateral amygdala. Proc Natl Acad Sci U S A 94, 14048-14053. 
Ralph, M.R., Foster, R.G., Davis, F.C., and Menaker, M. (1990). Transplanted suprachiasmatic nucleus determines circadian period. Science 247, 975-978.

Reischl, S., and Kramer, A. (2011). Kinases and phosphatases in the mammalian circadian clock. FEBS Lett 585, 1393-1399.

Roenneberg, T., Allebrandt, K.V., Merrow, M., and Vetter, C. (2012). Social jetlag and obesity. Curr Biol 22, 939-943.

Roenneberg, T., Kantermann, T., Juda, M., Vetter, C., and Allebrandt, K.V. (2013). Light and the human circadian clock. Handb Exp Pharmacol, 311-331.

Roenneberg, T., Wirz-Justice, A., and Merrow, M. (2003). Life between clocks: daily temporal patterns of human chronotypes. J Biol Rhythms 18, 80-90.

Romero, L.M. (2002). Seasonal changes in plasma glucocorticoid concentrations in free-living vertebrates. Gen Comp Endocrinol 128, 1-24.

Roozendaal, B., McReynolds, J.R., Van der Zee, E.A., Lee, S., McGaugh, J.L., and McIntyre, C.K. (2009). Glucocorticoid effects on memory consolidation depend on functional interactions between the medial prefrontal cortex and basolateral amygdala. J Neurosci 29, 14299-14308.

Roybal, K., Theobold, D., Graham, A., DiNieri, J.A., Russo, S.J., Krishnan, V., Chakravarty, S., Peevey, J., Oehrlein, N., Birnbaum, S., et al. (2007). Mania-like behavior induced by disruption of CLOCK. Proc Natl Acad Sci U S A 104, 6406-6411.

Rutter, J., Reick, M., Wu, L.C., and McKnight, S.L. (2001). Regulation of clock and NPAS2 DNA binding by the redox state of NAD cofactors. Science 293, 510-514.

Sage, D., Ganem, J., Guillaumond, F., Laforge-Anglade, G., Francois-Bellan, A.M., Bosler, O., and Becquet, D. (2004). Influence of the corticosterone rhythm on photic entrainment of locomotor activity in rats. J Biol Rhythms 19, 144-156.

Sage, D., Maurel, D., and Bosler, O. (2001). Involvement of the suprachiasmatic nucleus in diurnal ACTH and corticosterone responsiveness to stress. Am J Physiol Endocrinol Metab 280, E260-269.

Sage, D., Maurel, D., and Bosler, O. (2002). Corticosterone-dependent driving influence of the suprachiasmatic nucleus on adrenal sensitivity to ACTH. Am J Physiol Endocrinol Metab 282, E458465.

Sahar, S., and Sassone-Corsi, P. (2013). The epigenetic language of circadian clocks. Handb Exp Pharmacol, 29-44.

Saini, C., Morf, J., Stratmann, M., Gos, P., and Schibler, U. (2012). Simulated body temperature rhythms reveal the phase-shifting behavior and plasticity of mammalian circadian oscillators. Genes Dev 26, 567-580.

Salas, M.A., Evans, S.W., Levell, M.J., and Whicher, J.T. (1990). Interleukin-6 and ACTH act synergistically to stimulate the release of corticosterone from adrenal gland cells. Clin Exp Immunol $79,470-473$.

Sanghez, V., Razzoli, M., Carobbio, S., Campbell, M., McCallum, J., Cero, C., Ceresini, G., Cabassi, A., Govoni, P., Franceschini, P., et al. (2013). Psychosocial stress induces hyperphagia and exacerbates diet-induced insulin resistance and the manifestations of the Metabolic Syndrome. Psychoneuroendocrinology 38, 2933-2942. 
Sarabdjitsingh, R.A., Isenia, S., Polman, A., Mijalkovic, J., Lachize, S., Datson, N., de Kloet, E.R., and Meijer, O.C. (2010). Disrupted corticosterone pulsatile patterns attenuate responsiveness to glucocorticoid signaling in rat brain. Endocrinology 151, 1177-1186.

Sarabdjitsingh, R.A., Joels, M., and de Kloet, E.R. (2012). Glucocorticoid pulsatility and rapid corticosteroid actions in the central stress response. Physiol Behav 106, 73-80.

Sarlis, N.J., Chowdrey, H.S., Stephanou, A., and Lightman, S.L. (1992). Chronic activation of the hypothalamo-pituitary-adrenal axis and loss of circadian rhythm during adjuvant-induced arthritis in the rat. Endocrinology 130, 1775-1779.

Sato, T.K., Panda, S., Miraglia, L.J., Reyes, T.M., Rudic, R.D., McNamara, P., Naik, K.A., FitzGerald, G.A., Kay, S.A., and Hogenesch, J.B. (2004). A functional genomics strategy reveals Rora as a component of the mammalian circadian clock. Neuron 43, 527-537.

Scheer, F.A., Ter Horst, G.J., van Der Vliet, J., and Buijs, R.M. (2001). Physiological and anatomic evidence for regulation of the heart by suprachiasmatic nucleus in rats. Am J Physiol Heart Circ Physiol 280, H1391-1399.

Scheiermann, C., Kunisaki, Y., Lucas, D., Chow, A., Jang, J.E., Zhang, D., Hashimoto, D., Merad, M., and Frenette, P.S. (2012). Adrenergic nerves govern circadian leukocyte recruitment to tissues. Immunity 37, 290-301.

Schmidt, T.M., Chen, S.K., and Hattar, S. (2011). Intrinsically photosensitive retinal ganglion cells: many subtypes, diverse functions. Trends Neurosci 34, 572-580.

Segall, L.A., Milet, A., Tronche, F., and Amir, S. (2009). Brain glucocorticoid receptors are necessary for the rhythmic expression of the clock protein, PERIOD2, in the central extended amygdala in mice. Neurosci Lett 457, 58-60.

Sephton, S.E., Sapolsky, R.M., Kraemer, H.C., and Spiegel, D. (2000). Diurnal cortisol rhythm as a predictor of breast cancer survival. J Natl Cancer Inst 92, 994-1000.

Shanks, N., Windle, R.J., Perks, P.A., Harbuz, M.S., Jessop, D.S., Ingram, C.D., and Lightman, S.L. (2000). Early-life exposure to endotoxin alters hypothalamic-pituitary-adrenal function and predisposition to inflammation. Proc Natl Acad Sci U S A 97, 5645-5650.

Sheline, Y.I., Sanghavi, M., Mintun, M.A., and Gado, M.H. (1999). Depression duration but not age predicts hippocampal volume loss in medically healthy women with recurrent major depression. $\mathbf{J}$ Neurosci 19, 5034-5043.

Shi, P.A., Isola, L.M., Gabrilove, J.L., Moshier, E.L., Godbold, J.H., Miller, L.K., and Frenette, P.S. (2013). Prospective cohort study of the circadian rhythm pattern in allogeneic sibling donors undergoing standard granulocyte colony-stimulating factor mobilization. Stem Cell Res Ther 4, 30.

Shibli-Rahhal, A., Van Beek, M., and Schlechte, J.A. (2006). Cushing's syndrome. Clin Dermatol 24, 260-265.

Shimatsu, A., Kato, Y., Sakami, H., Nakai, Y., and Imura, H. (1988). Phase-shifted diurnal rhythm of cortisol secretion in a patient with Cushing's syndrome due to adrenocortical adenoma. Endocrinol Jpn $35,261-266$.

Shimizu, N., Oomura, Y., and Kai, Y. (1989). Stress-induced anorexia in rats mediated by serotonergic mechanisms in the hypothalamus. Physiol Behav 46, 835-841.

Shostak, A., Meyer-Kovac, J., and Oster, H. (2013a). Circadian regulation of lipid mobilization in white adipose tissues. Diabetes 62, 2195-2203. 
Shostak, A., Meyer-Kovac, J., and Oster, H. (2013b). Circadian regulation of lipid mobilization in white adipose tissues. Diabetes.

Silver, R., LeSauter, J., Tresco, P.A., and Lehman, M.N. (1996). A diffusible coupling signal from the transplanted suprachiasmatic nucleus controlling circadian locomotor rhythms. Nature 382, 810-813.

Simpson, E.R., and Waterman, M.R. (1988). Regulation of the synthesis of steroidogenic enzymes in adrenal cortical cells by ACTH. Annu Rev Physiol 50, 427-440.

So, A.Y., Bernal, T.U., Pillsbury, M.L., Yamamoto, K.R., and Feldman, B.J. (2009). Glucocorticoid regulation of the circadian clock modulates glucose homeostasis. Proc Natl Acad Sci U S A 106, 17582-17587.

Solberg, L.C., Horton, T.H., and Turek, F.W. (1999). Circadian rhythms and depression: effects of exercise in an animal model. Am J Physiol 276, R152-161.

Son, G.H., Chung, S., Choe, H.K., Kim, H.D., Baik, S.M., Lee, H., Lee, H.W., Choi, S., Sun, W., Kim, H., et al. (2008). Adrenal peripheral clock controls the autonomous circadian rhythm of glucocorticoid by causing rhythmic steroid production. Proc Natl Acad Sci U S A 105, 20970-20975.

Stavreva, D.A., Wiench, M., John, S., Conway-Campbell, B.L., McKenna, M.A., Pooley, J.R., Johnson, T.A., Voss, T.C., Lightman, S.L., and Hager, G.L. (2009). Ultradian hormone stimulation induces glucocorticoid receptor-mediated pulses of gene transcription. Nat Cell Biol 11, 1093-1102.

Stephan, F.K., and Zucker, I. (1972). Circadian rhythms in drinking behavior and locomotor activity of rats are eliminated by hypothalamic lesions. Proc Natl Acad Sci U S A 69, 1583-1586.

Sterling, P., and Eyer, J. (1981). Biological basis of stress-related mortality. Soc Sci Med E 15, 3-42.

Stocco, D.M. (2001). StAR protein and the regulation of steroid hormone biosynthesis. Annu Rev Physiol 63, 193-213.

Stokkan, K.A., Yamazaki, S., Tei, H., Sakaki, Y., and Menaker, M. (2001). Entrainment of the circadian clock in the liver by feeding. Science 291, 490-493.

Storch, K.F., Paz, C., Signorovitch, J., Raviola, E., Pawlyk, B., Li, T., and Weitz, C.J. (2007). Intrinsic circadian clock of the mammalian retina: importance for retinal processing of visual information. Cell $130,730-741$.

Storch, K.F., and Weitz, C.J. (2009). Daily rhythms of food-anticipatory behavioral activity do not require the known circadian clock. Proc Natl Acad Sci U S A 106, 6808-6813.

Stranahan, A.M. (2012). Chronobiological approaches to Alzheimer's disease. Curr Alzheimer Res 9, 93-98.

Stratmann, M., Suter, D.M., Molina, N., Naef, F., and Schibler, U. (2012). Circadian Dbp transcription relies on highly dynamic BMAL1-CLOCK interaction with $\mathrm{E}$ boxes and requires the proteasome. Mol Cell 48, 277-287.

Sujino, M., Masumoto, K.H., Yamaguchi, S., van der Horst, G.T., Okamura, H., and Inouye, S.T. (2003). Suprachiasmatic nucleus grafts restore circadian behavioral rhythms of genetically arrhythmic mice. Curr Biol 13, 664-668.

Sun, Y., Yang, Z., Niu, Z., Peng, J., Li, Q., Xiong, W., Langnas, A.N., Ma, M.Y., and Zhao, Y. (2006). MOP3, a component of the molecular clock, regulates the development of $\mathrm{B}$ cells. Immunology 119, 451-460. 
Surjit, M., Ganti, K.P., Mukherji, A., Ye, T., Hua, G., Metzger, D., Li, M., and Chambon, P. (2011). Widespread negative response elements mediate direct repression by agonist-liganded glucocorticoid receptor. Cell 145, 224-241.

Swiergiel, A.H., Leskov, I.L., and Dunn, A.J. (2008). Effects of chronic and acute stressors and CRF on depression-like behavior in mice. Behav Brain Res 186, 32-40.

Takahashi, K., Yamada, T., Tsukita, S., Kaneko, K., Shirai, Y., Munakata, Y., Ishigaki, Y., Imai, J., Uno, K., Hasegawa, Y., et al. (2013). Chronic mild stress alters circadian expressions of molecular clock genes in the liver. Am J Physiol Endocrinol Metab 304, E301-309.

Talaber, G., Jondal, M., and Okret, S. (2013). Extra-adrenal glucocorticoid synthesis: immune regulation and aspects on local organ homeostasis. Mol Cell Endocrinol 380, 89-98.

Tataroglu, O., Aksoy, A., Yilmaz, A., and Canbeyli, R. (2004). Effect of lesioning the suprachiasmatic nuclei on behavioral despair in rats. Brain Res 1001, 118-124.

Taves, M.D., Gomez-Sanchez, C.E., and Soma, K.K. (2011). Extra-adrenal glucocorticoids and mineralocorticoids: evidence for local synthesis, regulation, and function. Am J Physiol Endocrinol Metab 301, E11-24.

Terazono, H., Mutoh, T., Yamaguchi, S., Kobayashi, M., Akiyama, M., Udo, R., Ohdo, S., Okamura, H., and Shibata, S. (2003). Adrenergic regulation of clock gene expression in mouse liver. Proc Natl Acad Sci U S A 100, 6795-6800.

Thomas, W., and Harvey, B.J. (2011). Mechanisms underlying rapid aldosterone effects in the kidney. Annu Rev Physiol 73, 335-357.

Toh, K.L., Jones, C.R., He, Y., Eide, E.J., Hinz, W.A., Virshup, D.M., Ptacek, L.J., and Fu, Y.H. (2001). An hPer2 phosphorylation site mutation in familial advanced sleep phase syndrome. Science 291, 1040-1043.

Torres-Farfan, C., Seron-Ferre, M., Dinet, V., and Korf, H.W. (2006). Immunocytochemical demonstration of day/night changes of clock gene protein levels in the murine adrenal gland: differences between melatonin-proficient $(\mathrm{C} 3 \mathrm{H})$ and melatonin-deficient $(\mathrm{C} 57 \mathrm{BL})$ mice. J Pineal Res 40, 64-70.

Tosini, G., and Menaker, M. (1996). Circadian rhythms in cultured mammalian retina. Science 272, 419-421.

Tsujimura, S., Saito, K., Nawata, M., Nakayamada, S., and Tanaka, Y. (2008). Overcoming drug resistance induced by P-glycoprotein on lymphocytes in patients with refractory rheumatoid arthritis. Ann Rheum Dis 67, 380-388.

Turek, F.W. (2007). From circadian rhythms to clock genes in depression. Int Clin Psychopharmacol 22 Suppl 2, S1-8.

Turek, F.W., Joshu, C., Kohsaka, A., Lin, E., Ivanova, G., McDearmon, E., Laposky, A., Losee-Olson, S., Easton, A., Jensen, D.R., et al. (2005). Obesity and metabolic syndrome in circadian Clock mutant mice. Science 308, 1043-1045.

Ueda, H.R., Hayashi, S., Chen, W., Sano, M., Machida, M., Shigeyoshi, Y., Iino, M., and Hashimoto, S. (2005). System-level identification of transcriptional circuits underlying mammalian circadian clocks. Nat Genet 37, 187-192. 
Uhlenhaut, N.H., Barish, G.D., Yu, R.T., Downes, M., Karunasiri, M., Liddle, C., Schwalie, P., Hubner, N., and Evans, R.M. (2013). Insights into negative regulation by the glucocorticoid receptor from genome-wide profiling of inflammatory cistromes. Mol Cell 49, 158-171.

Ulrich-Lai, Y.M., Arnhold, M.M., and Engeland, W.C. (2006). Adrenal splanchnic innervation contributes to the diurnal rhythm of plasma corticosterone in rats by modulating adrenal sensitivity to ACTH. Am J Physiol Regul Integr Comp Physiol 290, R1128-1135.

Ulrich-Lai, Y.M., and Engeland, W.C. (2000). Rat adrenal transplants are reinnervated: an invalid model of denervated adrenal cortical tissue. J Neuroendocrinol 12, 881-893.

Ulrich-Lai, Y.M., and Engeland, W.C. (2002). Adrenal splanchnic innervation modulates adrenal cortical responses to dehydration stress in rats. Neuroendocrinology 76, 79-92.

Ulrich-Lai, Y.M., and Herman, J.P. (2009). Neural regulation of endocrine and autonomic stress responses. Nat Rev Neurosci 10, 397-409.

Valenzuela, F.J., Torres-Farfan, C., Richter, H.G., Mendez, N., Campino, C., Torrealba, F., Valenzuela, G.J., and Seron-Ferre, M. (2008). Clock gene expression in adult primate suprachiasmatic nuclei and adrenal: is the adrenal a peripheral clock responsive to melatonin? Endocrinology 149, 1454-1461.

Valles, A., Marti, O., Garcia, A., and Armario, A. (2000). Single exposure to stressors causes longlasting, stress-dependent reduction of food intake in rats. Am J Physiol Regul Integr Comp Physiol 279, R1138-1144.

van der Horst, G.T., Muijtjens, M., Kobayashi, K., Takano, R., Kanno, S., Takao, M., de Wit, J., Verkerk, A., Eker, A.P., van Leenen, D., et al. (1999). Mammalian Cry1 and Cry2 are essential for maintenance of circadian rhythms. Nature 398, 627-630.

Vegiopoulos, A., and Herzig, S. (2007). Glucocorticoids, metabolism and metabolic diseases. Mol Cell Endocrinol 275, 43-61.

Volden, P.A., and Conzen, S.D. (2013). The influence of glucocorticoid signaling on tumor progression. Brain Behav Immun 30 Suppl, S26-31.

Vollmers, C., Gill, S., DiTacchio, L., Pulivarthy, S.R., Le, H.D., and Panda, S. (2009). Time of feeding and the intrinsic circadian clock drive rhythms in hepatic gene expression. Proc Natl Acad Sci U S A 106, 21453-21458.

Vujovic, N., Davidson, A.J., and Menaker, M. (2008). Sympathetic input modulates, but does not determine, phase of peripheral circadian oscillators. Am J Physiol Regul Integr Comp Physiol 295, R355-360.

Wagner, U., and Born, J. (2008). Memory consolidation during sleep: interactive effects of sleep stages and HPA regulation. Stress 11, 28-41.

Wagner, U., Degirmenci, M., Drosopoulos, S., Perras, B., and Born, J. (2005). Effects of cortisol suppression on sleep-associated consolidation of neutral and emotional memory. Biol Psychiatry 58, 885-893.

Waite, E.J., McKenna, M., Kershaw, Y., Walker, J.J., Cho, K., Piggins, H.D., and Lightman, S.L. (2012). Ultradian corticosterone secretion is maintained in the absence of circadian cues. Eur $\mathbf{J}$ Neurosci 36, 3142-3150.

Walker, J.J., Terry, J.R., and Lightman, S.L. (2010). Origin of ultradian pulsatility in the hypothalamic-pituitary-adrenal axis. Proc Biol Sci 277, 1627-1633. 
Weaver, I.C., Cervoni, N., Champagne, F.A., D'Alessio, A.C., Sharma, S., Seckl, J.R., Dymov, S., Szyf, M., and Meaney, M.J. (2004). Epigenetic programming by maternal behavior. Nat Neurosci 7, 847-854.

Welsh, D.K., Takahashi, J.S., and Kay, S.A. (2010). Suprachiasmatic nucleus: cell autonomy and network properties. Annu Rev Physiol 72, 551-577.

Welsh, D.K., Yoo, S.H., Liu, A.C., Takahashi, J.S., and Kay, S.A. (2004). Bioluminescence imaging of individual fibroblasts reveals persistent, independently phased circadian rhythms of clock gene expression. Curr Biol 14, 2289-2295.

Windle, R.J., Wood, S.A., Kershaw, Y.M., Lightman, S.L., Ingram, C.D., and Harbuz, M.S. (2001). Increased corticosterone pulse frequency during adjuvant-induced arthritis and its relationship to alterations in stress responsiveness. J Neuroendocrinol 13, 905-911.

Windle, R.J., Wood, S.A., Shanks, N., Lightman, S.L., and Ingram, C.D. (1998). Ultradian rhythm of basal corticosterone release in the female rat: dynamic interaction with the response to acute stress. Endocrinology 139, 443-450.

Xu, C., He, J., Jiang, H., Zu, L., Zhai, W., Pu, S., and Xu, G. (2009). Direct effect of glucocorticoids on lipolysis in adipocytes. Mol Endocrinol 23, 1161-1170.

Xu, R.B., Liu, Z.M., and Zhao, Y. (1991). A study on the circadian rhythm of glucocorticoid receptor. Neuroendocrinology 53 Suppl 1, 31-36.

Xu, Y., Padiath, Q.S., Shapiro, R.E., Jones, C.R., Wu, S.C., Saigoh, N., Saigoh, K., Ptacek, L.J., and Fu, Y.H. (2005). Functional consequences of a CKIdelta mutation causing familial advanced sleep phase syndrome. Nature 434, 640-644.

Yamaguchi, Y., Suzuki, T., Mizoro, Y., Kori, H., Okada, K., Chen, Y., Fustin, J.M., Yamazaki, F., Mizuguchi, N., Zhang, J., et al. (2013). Mice genetically deficient in vasopressin V1a and V1b receptors are resistant to jet lag. Science 342, 85-90.

Yamamoto, T., Nakahata, Y., Tanaka, M., Yoshida, M., Soma, H., Shinohara, K., Yasuda, A., Mamine, T., and Takumi, T. (2005). Acute physical stress elevates mouse period1 mRNA expression in mouse peripheral tissues via a glucocorticoid-responsive element. J Biol Chem 280, 42036-42043.

Yamazaki, S., Numano, R., Abe, M., Hida, A., Takahashi, R., Ueda, M., Block, G.D., Sakaki, Y., Menaker, M., and Tei, H. (2000). Resetting central and peripheral circadian oscillators in transgenic rats. Science 288, 682-685.

Yaneva, M., Mosnier-Pudar, H., Dugue, M.A., Grabar, S., Fulla, Y., and Bertagna, X. (2004). Midnight salivary cortisol for the initial diagnosis of Cushing's syndrome of various causes. J Clin Endocrinol Metab 89, 3345-3351.

Yang, M.Y., Chang, J.G., Lin, P.M., Tang, K.P., Chen, Y.H., Lin, H.Y., Liu, T.C., Hsiao, H.H., Liu, Y.C., and Lin, S.F. (2006). Downregulation of circadian clock genes in chronic myeloid leukemia: alternative methylation pattern of hPER3. Cancer Sci 97, 1298-1307.

Yang, S., Liu, A., Weidenhammer, A., Cooksey, R.C., McClain, D., Kim, M.K., Aguilera, G., Abel, E.D., and Chung, J.H. (2009). The role of mPer2 clock gene in glucocorticoid and feeding rhythms. Endocrinology 150, 2153-2160.

Yehuda, R., Golier, J.A., and Kaufman, S. (2005). Circadian rhythm of salivary cortisol in Holocaust survivors with and without PTSD. Am J Psychiatry 162, 998-1000. 
Yi, C.X., Foppen, E., Abplanalp, W., Gao, Y., Alkemade, A., la Fleur, S.E., Serlie, M.J., Fliers, E., Buijs, R.M., Tschop, M.H., et al. (2012). Glucocorticoid signaling in the arcuate nucleus modulates hepatic insulin sensitivity. Diabetes 61, 339-345.

Yoo, S.H., Yamazaki, S., Lowrey, P.L., Shimomura, K., Ko, C.H., Buhr, E.D., Siepka, S.M., Hong, H.K., Oh, W.J., Yoo, O.J., et al. (2004). PERIOD2::LUCIFERASE real-time reporting of circadian dynamics reveals persistent circadian oscillations in mouse peripheral tissues. Proc Natl Acad Sci U S A $101,5339-5346$.

Yu, E.A., and Weaver, D.R. (2011). Disrupting the circadian clock: gene-specific effects on aging, cancer, and other phenotypes. Aging (Albany NY) 3, 479-493.

Zhang, E.E., and Kay, S.A. (2010). Clocks not winding down: unravelling circadian networks. Nat Rev Mol Cell Biol 11, 764-776.

Zhang, J., Wu, Z., Zhou, L., Li, H., Teng, H., Dai, W., Wang, Y., and Sun, Z.S. (2011). Deficiency of antinociception and excessive grooming induced by acute immobilization stress in Per1 mutant mice. PLoS One 6, e16212.

Zheng, B., Albrecht, U., Kaasik, K., Sage, M., Lu, W., Vaishnav, S., Li, Q., Sun, Z.S., Eichele, G., Bradley, A., et al. (2001). Nonredundant roles of the mPer1 and mPer2 genes in the mammalian circadian clock. Cell 105, 683-694.

Zhou, J.N., Riemersma, R.F., Unmehopa, U.A., Hoogendijk, W.J., van Heerikhuize, J.J., Hofman, M.A., and Swaab, D.F. (2001). Alterations in arginine vasopressin neurons in the suprachiasmatic nucleus in depression. Arch Gen Psychiatry 58, 655-662.

Zobel, A.W., Nickel, T., Sonntag, A., Uhr, M., Holsboer, F., and Ising, M. (2001). Cortisol response in the combined dexamethasone/CRH test as predictor of relapse in patients with remitted depression. a prospective study. J Psychiatr Res 35, 83-94. 


\section{Curriculum Vitae}

Name: $\quad$ Alexei Leliavski

Date and place of birth: $\quad 02.08 .1984$, Minsk, Belarus

Citizenship: $\quad$ Republic of Belarus

\section{Education}

2002-2007 — study of biology at the International Sakharov Environmental University (ISEU) in Minsk, Belarus;

2007 - Diploma thesis in immunology; the title "Functional activity of peripheral blood mononuclear cells in patients with secondary osteoporosis";

2008 - Master's thesis in genetics (one-year 'Magister' degree);

2008-2009 - a participant of the Program in Oncoimmunology (Moscow, Russia; http://www.oncoimmunology.ru );

2010-2013 - doctoral studies in the Chronophysiology Group (Prof. Dr. Henrik Oster) at University of Lübeck, Germany (formerly the Circadian Rhythms Group, Max Planck Institute for Biophysical Chemistry, Göttingen); the doctoral program „Genes and Development“ in the Göttingen Graduate School for Neurosciences and Molecular Biosciences (GGNB). The project title: "Physiological functions of the adrenocortical circadian clock".

\section{Work experience}

March 2008 - Dec 2009: Research assistant at the Belarusian Research Center for Pediatric Oncology and Hematology;

Sept 2008 - Sept 2011: News articles for the Cellular Transplantation and Tissue Engineering journal (in Russian; http://celltranspl.ru ).

\section{Grants and Awards}

2010-20012 - the two-year Gerhardt Hunsmann Fellowship for doctoral students

Der Lübecker Doktorandentag “Uni im Dialog”, June 19, 2013; „Stressresistenz in Mäusen ohne innere Uhr" (a poster prize of the year 2013) 


\section{Publications}

Leliavski A, Oster H (2014) Physiology of the adrenal and liver circadian clocks; In: Circadian Medicine (Wiley-Blackwell, Ed.: C.S. Colwell), in press.

Contribution: writing a part about the adrenal circadian clock.

Leliavski A, Shostak A, Husse J, Oster H (2013) Impaired Glucocorticoid Production and Response to Stress in Arntl-Deficient Male Mice. Endocrinology. Nov 4. en.2013-1531.

Contribution: performing all the experiments included in the paper, data analysis, manuscript writing.

Husse J, Leliavski A, Oster H (2013) Biological role and clinical relevance of central and peripheral circadian clocks. Dtsch Med Wochenschr. 138(10):493-6.

Contribution: providing data and preparing Figure 2.

Barclay JL, Shostak A, Leliavski A, Tsang AH, Jöhren O, Müller-Fielitz H, Landgraf D, Naujokat N, van der Horst GT, Oster H (2013) High-fat diet-induced hyperinsulinemia and tissue-specific insulin resistance in Cry-deficient mice. Am J Physiol Endocrinol Metab. 304(10):E1053-63.

Contribution: performing hormonal and lipid analyses (Supplemetary table), data analysis, manuscript writing.

Chu A, Zhu L, Blum ID, Mai O, Leliavski A, Fahrenkrug J, Oster H, Boehm U, Storch KF (2013) Global but not gonadotrope-specific disruption of Bmal1 abolishes the luteinizing hormone surge without affecting ovulation. Endocrinology. 154(8):2924-35.

Contribution: performing luminescence imaging of pituitary explants (Figure 1B), data analysis, figure preparation, manuscript writing.

Barclay JL*, Leliavski AA*, Oster H (2012) Circadian Clocks and Eating Disorders; In: Circadian Rhythms, Eds.: L. Golovkin \& A. Maliszkewicz; pp.1-27. (* equal contribution)

Contribution: writing an introductory part on physiology and circadian rhythms of appetite regulation (pp. 2-9).

Bollinger T, Leutz A, Leliavski A, Skrum L, Kovac J, Bonacina L, Benedict C, Lange T, Westermann J, Oster H, Solbach W. (2011) Circadian clocks in mouse and human CD4+ T cells. PLoS One. 6(12):e29801.

Contribution: performing luminescence imaging of thymus explants (Figure 1) and unpublished experiments for the project, data analysis, figure preparation, manuscript writing. 


\section{Acknowledgements}

First of all, I would like to thank my supervisor, Henrik Oster, for his enormous everyday support, trust, humor and patience over these amazing four years in his group that became a unique and extraordinary experience for me. His door in the office as well as his mind was and is always open to any suggestions, needs and ideas. Henrik provided a wonderful chance to me to explore and learn, and became a beautiful example of motivation and determination in science.

I want to thank the members of my thesis committee, Michael Kessel and Andreas Wodarz, for constructive and encouraging suggestions. They were always helpful, kind and optimistic, even at tough turns of the project.

I am particularly grateful to Gerhard Hunsmann, who kindly provided a financial support to me over the first two years of my $\mathrm{PhD}$. His help and trust was essential for me to join Henrik's group and start the project .

I would like to express my gratitude to Gregor Eichele and all members of Genes and Behavior Department for daily support, lovely environment and fruitful working atmosphere. Many thanks to Helena Miletic and Christine van den Bogaart for considerable organizational support and our animal caretakers and technicians Nadine Naujokat, Christin Helbig, Alexandra Driehorst and Kesrtin Luensmann for continuous help and patience.

Thanks to all current and former members of the Circadian Rhythms Group, your everyday help, suggestions, experimental advices and enthusiasm were invaluable for me! Thank you so much, Judit, Jana, Anthony, Johanna, Anton, Christiane, Dominic, Brid, Roberta, Silke - you all are amazing colleagues and friends!

And, of course, I want to thank people that helped me start and keep going at our new place, in Lübeck. I'm especially grateful to Volker Ott and Olaf Jöhren, who helped me with some critical experiments at the end of my project.

Finally, I thank my family - Olya and Ilya, who have gone with me through this experience, sharing love, joy and troubles, encouraging and motivating. And waiting, waiting for years. I love you both so much!

Alexei 